\title{
Compartment Venting Analyses of Ares I First Stage Systems Tunnel
}

\author{
Qunzhen Wang ${ }^{1}$ and Stephen Arner ${ }^{2}$ \\ ATK Space Systems, Brigham City, UT 84302
}

\begin{abstract}
Compartment venting analyses have been performed for the Ares I first stage systems tunnel using both the lumped parameter method and the three-dimensional (3D) transient computational fluid dynamics (CFD) approach. The main objective of venting analyses is to predict the magnitudes of differential pressures across the skin so the integrity of solid walls can be evaluated and properly designed. The lumped parameter method assumes the gas pressure and temperature inside the systems tunnel are spatially uniform, which is questionable since the tunnel is about 1,700 in. long and $4 \mathrm{in}$. wide. Therefore, $3 \mathrm{D}$ transient CFD simulations using the commercial CFD code FLUENT are performed in order to examine the gas pressure and temperature variations inside the tunnel. It was found that the uniform pressure and temperature assumptions inside the systems tunnel are valid during ascent. During reentry, the uniform pressure assumption is also reasonable but the uniform temperature assumption is not valid. Predicted pressure and temperature inside the systems tunnel using CFD are also compared with those from the lumped parameter method using the NASA code CHCHVENT. In general, the average pressure and temperature inside the systems tunnel from CFD are between the burst and crush results from CHCHVENT during both ascent and reentry. The skin differential pressure and pressure inside the systems tunnel relative to freestream pressure from CHCHVENT as well as velocity vectors and streamlines are also discussed in detail.
\end{abstract}

\section{Introduction}

Several compartments in the Ares I Crew Launch Vehicle contain trapped gases that will escape through vents and/or leak paths during flight. During ascent, the vehicle freestream experiences a decrease in atmospheric pressure. The pressures inside the vented compartments also decrease, but lag the freestream pressure. Both the freestream pressure and pressure inside the compartments increase during reentry, but the internal pressure again lags the freestream pressure. Thus, differential pressures across the vehicle walls are induced through all phases of flight. These differential pressures can be partially controlled by vent size and location. The magnitudes of differential pressures need to be quantified by performing venting analyses so the integrity of solid walls can be evaluated and properly designed.

Compartment venting analyses are usually performed using the lumped parameter method ${ }^{1-4}$ by assuming the gas pressure and temperature inside the compartment are spatially uniform and the gas velocity is zero. This approach is termed zero-dimensional (0D) because the pressure and temperature of the gas inside the compartments do not change with location at any instant during the transient process. The gas flow between the compartment and the freestream through the vent is assumed to be one-dimensional (1D), isentropic, and quasi-steady. The gas is assumed to be inviscid and an empirical discharge coefficient, which depends on the pressure ratio across the vent and the crossflow Mach number, is applied to account for viscous and other losses.

The purpose of this paper is to document the compartment venting analyses of the Ares I first stage systems tunnel during both ascent and reentry. As shown in Figure 1, the Ares I first stage systems tunnel begins at the forward skirt and ends at the aft skirt. This tunnel provides a protective housing for critical pyrotechnic charges, electrical wiring, and pneumatic tubing that run along the exterior surface of the first stage and shields these components from adverse environmental conditions that can lead to system failure. To ensure that the systems tunnel maintains its structural integrity during flight, venting analyses need to be performed to predict the maximum burst and crush pressure differentials that could be expected across the tunnel walls. Venting analyses of other

\footnotetext{
${ }^{1}$ Sr. Engineering Specialist, Fluid Dynamics, M/S 252, AIAA senior member.

${ }^{2}$ Project Engineer, Ares Systems Engineering, M/S L00.
} 
compartments such as forward skirt extension, aeroshell, and aft skirt are also performed using a similar procedure ${ }^{1-2}$ but are not discussed in this paper. The tool used to perform venting analyses is a computer program called CHCHVENT $^{5}$. The pressure coefficients used to calculate local pressures during ascent are obtained from wind tunnel testing ${ }^{6}$ while those from CFD predictions are used for the reentry venting analyses. A total of 1,000 trajectories are used to predict the worst burst and crush skin differential pressures during both ascent and reentry.

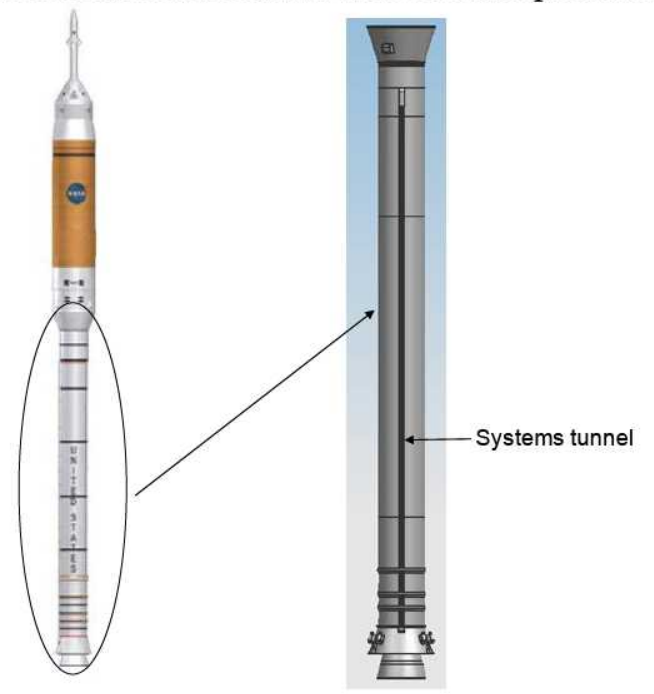

Figure 1. Ares I first stage systems tunnel.

As discussed above, the 0D lumped parameter method does not account for the pressure and temperature variation inside the compartment. This is especially relevant to the Ares I first stage systems tunnel that is about $1,700 \mathrm{in.} \mathrm{long}$ and $4 \mathrm{in}$. wide. It is questionable to assume uniform pressure and temperature inside such a long tunnel. Therefore, 3D transient CFD predictions using the commercial CFD code FLUENT are performed in order to examine the gas pressure and temperature variations inside the tunnel. Details such as the time history of maximum, minimum, and average pressure and temperature inside the tunnel as well as velocity vectors near the vent are presented in this paper. In addition, results from CFD are compared with those from the lumped parameter method.

\section{0D Lumped Parameter Analyses}

The 0D lumped parameter method tool used to perform the venting analyses is a FORTRAN computer program called CHCHVENT, which was developed at NASA and documented in Ref. 5. CHCHVENT models onedimensional (1D) flow among an arbitrary arrangement of compartments and vents. Inside the compartment, the pressure and temperature are assumed to be uniform (i.e., no variation with location) and the velocity is assumed to be zero. Mass and energy fluxes into and out of each compartment are computed using the current thermodynamic state of each compartment and then numerically integrated over a small time interval to update the state of each compartment. The results produced are time histories of the gas pressure, temperature, and density in each compartment and the mass flow rates through each vent. Initial pressure, temperature, and volume are the basic required inputs for the compartments. Vent area, network connectivity, and discharge coefficient model are the necessary inputs for the vents.

According to the $1 \mathrm{D}$ isentropic flow theory of a perfect gas, the Mach number at the vent can be calculated as

$$
M=\sqrt{\frac{2}{\gamma-1}\left[\left(\frac{P_{U}}{P_{D}}\right)^{\frac{\gamma-1}{\gamma}}-1\right]}
$$

where 8 is the ratio of specific heats while $P_{U}$ and $P_{D}$ are the upstream and downstream pressures, respectively. If Eq. 1 yields a Mach number larger than unity, the flow in the vent is choked and the Mach number is set to one. The mass flow rate through the vent is then calculated from the Mach number as 


$$
\dot{m}=C_{D} A P_{U} \sqrt{\frac{\gamma}{R T_{U}}} \frac{M}{\left[1+\frac{\gamma-1}{2} M^{2}\right]^{\frac{\gamma+1}{2(\gamma-1)}}}
$$

where $C_{D}$ is the vent discharge coefficient, $A$ is the area of the vent, $R$ is the gas constant, and $T_{U}$ is the upstream stagnation temperature. The upstream pressure $P_{U}$ and downstream pressure $P_{D}$ can be either the compartment pressure $P_{C}$ or the local external pressure at the vent $P_{L}$, which is calculated as

$$
p_{L}(t)=C p\left(M_{\infty}, \theta, \varphi\right) \times q_{\infty}(t)+p_{\infty}(t)
$$

where $C p$ is the pressure coefficient, $\theta$ is the pitch angle, and $\varphi$ is the roll angle. The dynamic pressure can be calculated from the ambient pressure and Mach number as

$$
q_{\infty}=\frac{1}{2} \gamma p_{\infty} M_{\infty}^{2}
$$

Since the freestream pressure $p_{\infty}$ and Mach number $M_{\infty}$ as well as pitch and roll angles are determined from the vehicle trajectory data, the most important input parameters in venting analyses are trajectories, pressure coefficients, and discharge coefficients, which are discussed separately in the next three sections.

\section{A. Trajectories}

A total of 1,000 trajectories are used in the CHCHVENT venting analyses during both ascent and reentry. Figure 2 and Figure 3 show the pressure and Mach number of the Ares I mean trajectory during ascent and reentry, respectively. During ascent, the pressure decreases from 14.7 psia to almost zero after 120 sec whereas the Mach number increases from zero to about 5.7. On the other hand, the pressure increases and the Mach number decreases during reentry.
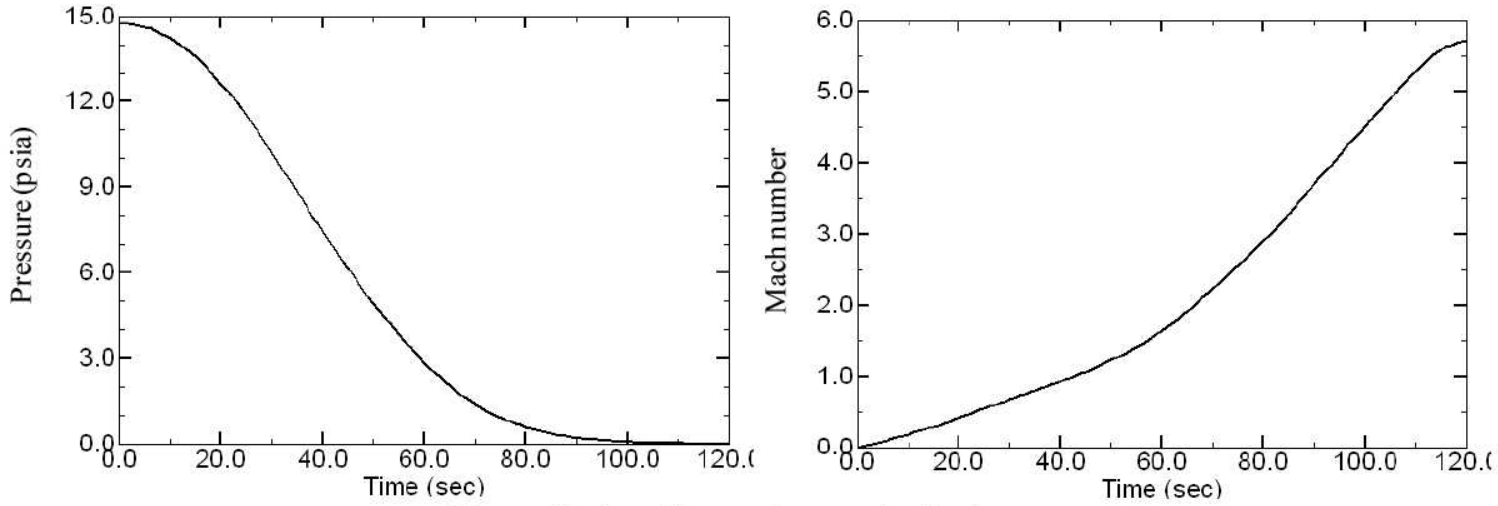

Figure 2. Ares I ascent mean trajectory.
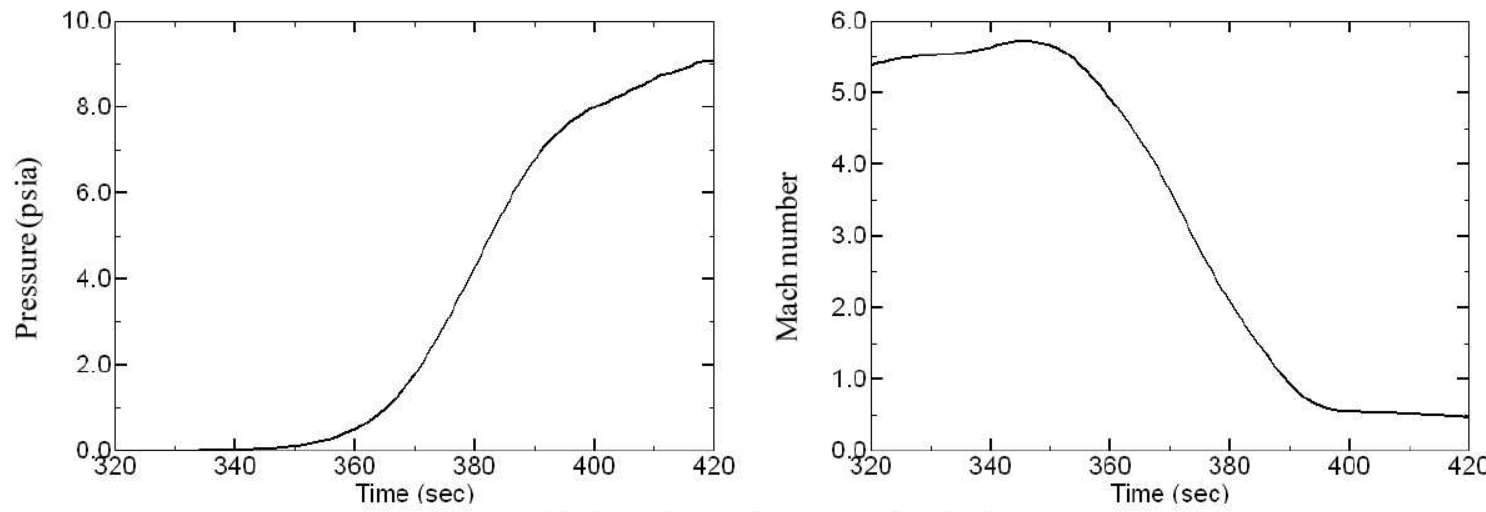

Figure 3. Ares I reentry mean trajectory.

The pitch angles of the Ares I trajectories during both ascent and reentry are shown in Figure 4. During ascent, the pitch angles are smaller than five degrees except for the first few seconds whereas the pitch angles are much larger during reentry. 

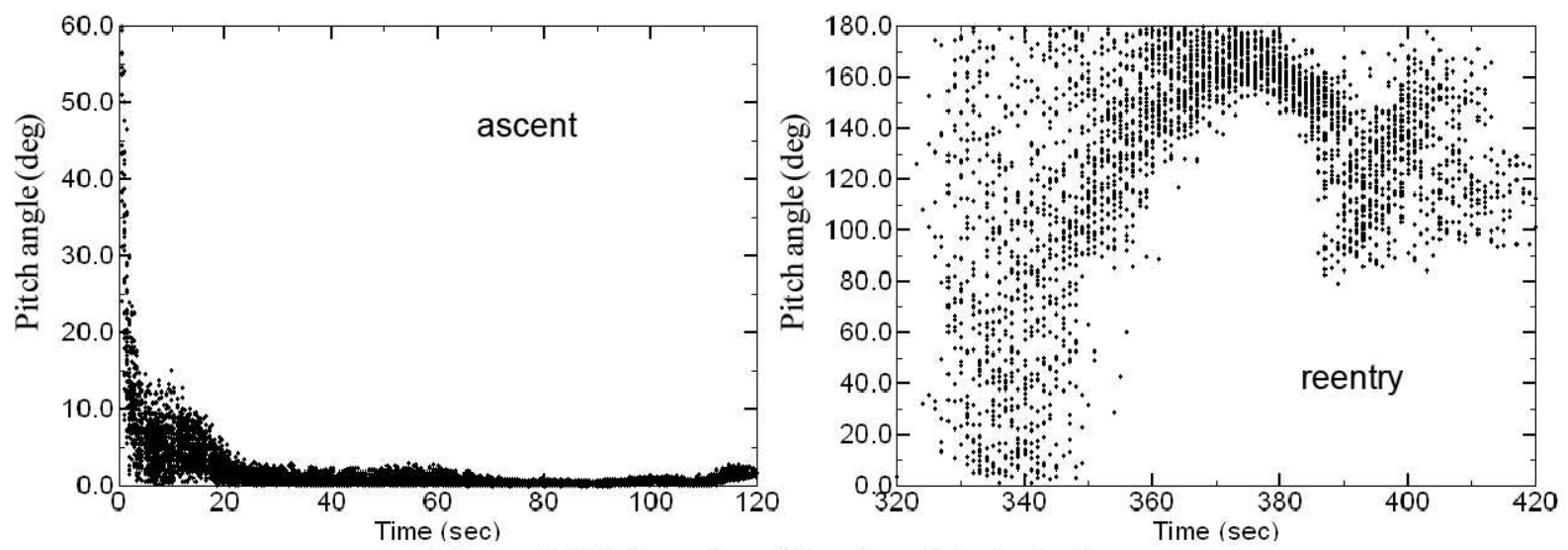

Figure 4. Pitch angles of the Ares I trajectories.

\section{B. Pressure Coefficients}

The pressure coefficient data from the Ares I wind tunnel testing ${ }^{6}$ are used for ascent venting analyses. Pressure tests were performed using a one percent scale model of the Ares I vehicle configuration over the Mach number range from 0.5 to 4.5 (20 Mach numbers total) and pitch angles from 0 to 7 degrees (four total). The test model was instrumented with 146 pressure taps at the surface of the vehicle as depicted in Figure 5.

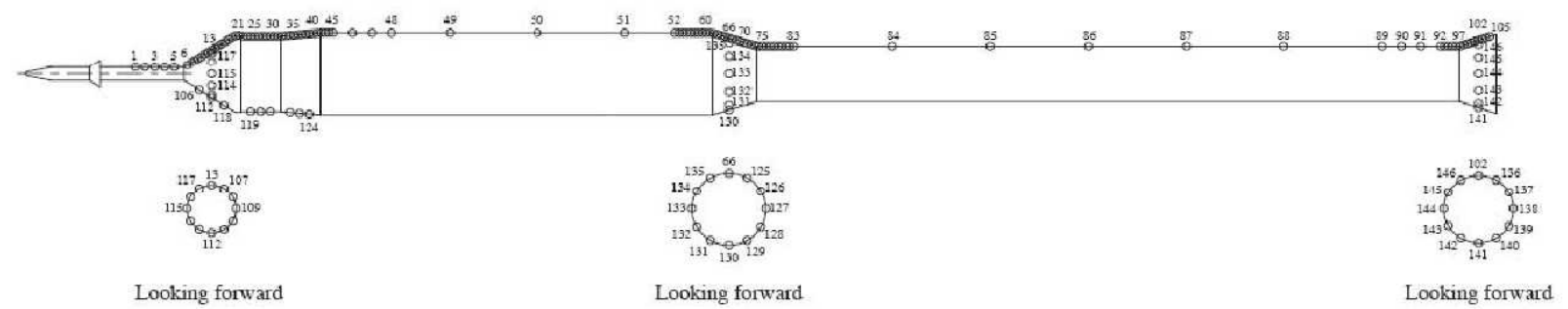

Figure 5. Ares I wind tunnel pressure test model.

CFD models of the Ares I first stage clean skin configuration (no protuberance) were built and the pressure coefficient using the CFD code CFD++ was applied in the reentry venting analyses ${ }^{2}$. The steady-state CFD simulations were run with the realizable k- 4 turbulence model and wall functions. All solutions were second-order accurate and were run on a grid with approximately 4.8 million cells. Pressure coefficients at four Mach numbers $(0.5,0.95,1.05$, and 4.0$)$ and six pitch angles $(0,45,90,120,150$, and 180 degrees) were obtained. Figure 6 shows the geometry of the Ares I first stage vehicle used in the CFD model.

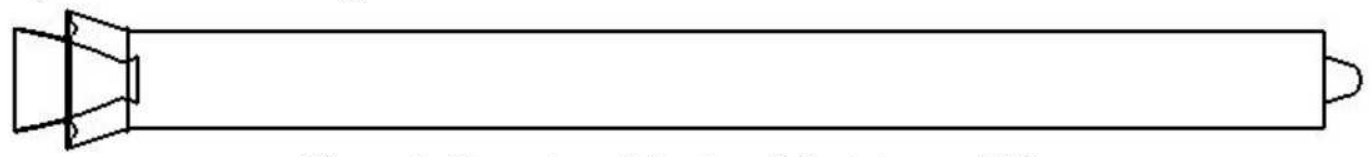

Figure 6. Geometry of the Ares I first stage vehicle.

\section{Discharge Coefficients}

As shown in Eq. (2), CHCHVENT computes the mass flow rate through a vent as the product of the ideal (inviscid) theoretical mass flow rate and a discharge coefficient to account for viscous and other losses. Two orifice discharge coefficient models were used in this paper: (1) a pressure dependent model that depends on the pressure ratio only and (2) a crossflow dependent model that depends on both pressure ratio and freestream Mach number.

As discussed in Ref. 3, a series of wind-tunnel tests were conducted to measure the outflow and inflow discharge coefficients for orifices with different pressure ratios and crossflow Mach numbers. The experimental data points were plotted and minimum and maximum bounding curves were drawn around them. These curves were combined with no-crossflow pressure dependent discharge coefficient data $(\mathrm{M}=0)$ and translated into tables that are known as K-files. As shown in Figure 7, K-file 1 (KF1) contains the minimum values of the inflow discharge coefficients and the maximum values of the outflow discharge coefficients and thus yields the lowest chamber pressures. On the other hand, K-file 2 (KF2) contains the maximum values of the inflow discharge coefficients and the minimum values of the outflow discharge coefficients and thus yields the highest chamber pressures. Note that gas flows out 
of the compartment when the pressure inside the compartment $P c$ is larger than the local external pressure $P_{L}$ (i.e., $P c / P_{L}>1$ ) whereas inflow occurs when $P_{c} / P_{L}<1$. Figure 8 compares the magnitudes of KF1 and KF2 at the same crossflow Mach number and pressure ratios. As expected, KF1 is smaller than KF2 when the flow is from ambient to compartment $\left(P c / P_{L}<1\right)$ whereas $\mathrm{KF} 1$ is larger than KF2 when the flow is from compartment to ambient $\left(P c / P_{L}\right.$ $>1$ ). Except for KF1 at very small Mach numbers, the discharge coefficient decreases with the increasing crossflow Mach number for the same pressure ratio indicating that crossflow reduces orifice efficiency. At the same crossflow Mach number, the discharge coefficient decreases when the pressure ratio is closer to one.
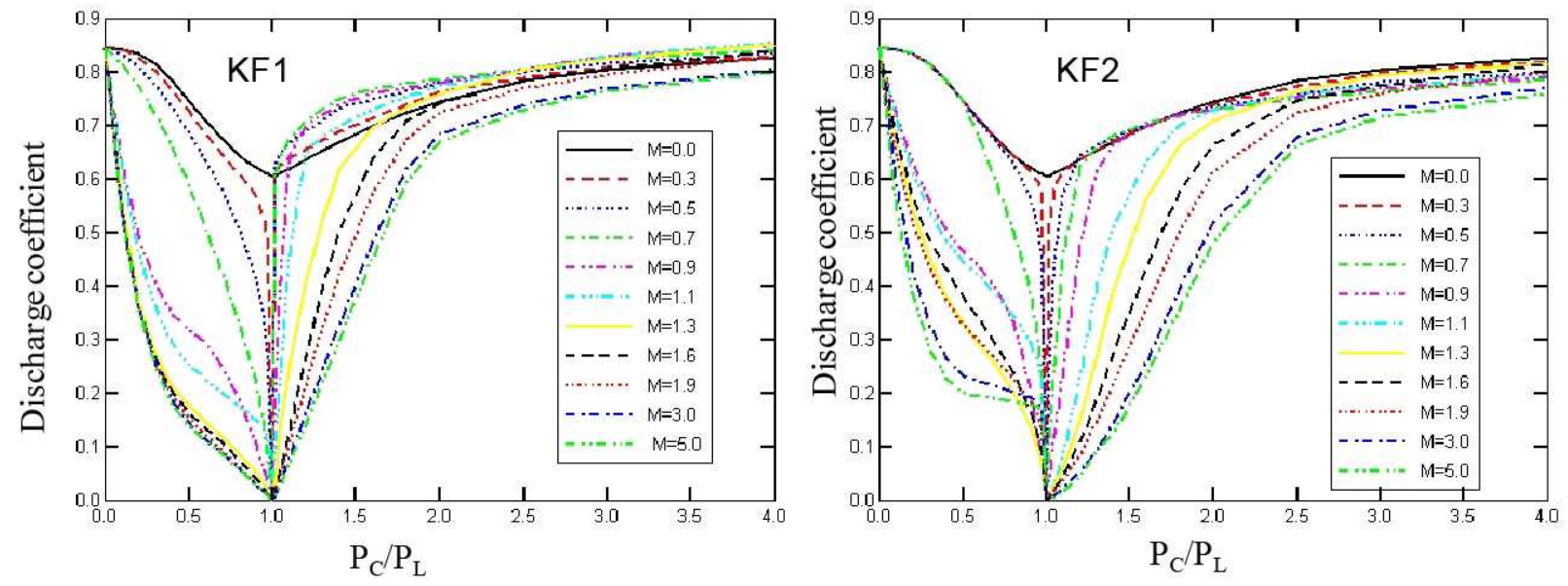

Figure 7. Crossflow dependent discharge coefficients. KF1 is for minimum compartment pressure whereas KF2 is for maximum compartment pressure.
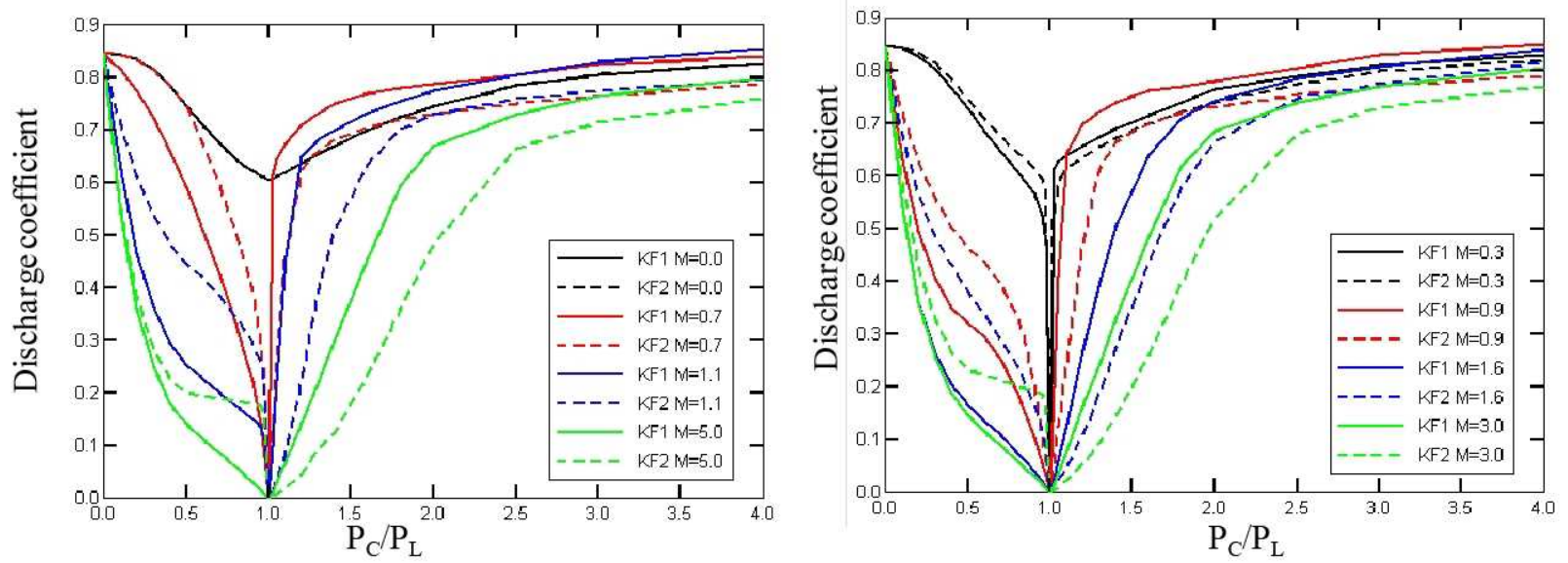

Figure 8. Comparison of crossflow dependent discharge coefficients KF1 and KF2.

\section{Burst Versus Crush}

Different modeling assumptions are used to predict the worst case skin differential pressures, which are defined as the difference between pressure inside the compartment and the local external pressure at the wall:

$$
d p_{\text {skin }}(t, x)=p_{C}(t)-C p\left(M_{\infty}, \theta, \varphi, x\right) \times q_{\infty}(t)-p_{\infty}(t)
$$

For the burst case corresponding to maximum skin differential pressure, venting models were created to maximize the internal compartment pressures during flight. Minimum external wall pressures were coupled to these models to provide maximum burst loads. For the crush case corresponding to minimum skin differential pressure, models providing minimum internal compartment pressures were coupled with maximum external wall pressures.

As shown in Table 1, two ascent venting models are created for each trajectory. For the burst case, the minimum discharge coefficient should be used, which corresponds to XF2 since, as shown in Figure 7, crossflow reduces the orifice efficiency. The maximum discharge coefficient corresponding to the pressure dependent model is used for the crush case. The pressure coefficient at the vent is a function of Mach number, pitch angle, and roll angle. However, the pressure coefficient used to calculate the external wall pressure is the minimum over all roll angles for the burst case and maximum for the crush case. The heat transfer between gas inside the compartment and solid 
walls is modeled in CHCHVENT to be either adiabatic, where the heat transfer is neglected, or isothermal, where the gas temperature is assumed to be constant at the initial temperature. The isothermal assumption is used for the burst case whereas the adiabatic assumption is used for the crush case.

Table 1. Run matrix for ascent venting analyses.

\begin{tabular}{|c|c|c|c|}
\hline & Thermal & $\mathrm{Cd}$ & Wall $\mathrm{cp}$ \\
\hline burst & isothermal & $\mathrm{XF2}$ & $\min \mathrm{f}(\mathrm{M}$,theta $)$ \\
\hline crush & adiabatic & Pdep & $\max \mathrm{f}(\mathrm{M}$, theta $)$ \\
\hline
\end{tabular}

The two venting models created for each trajectory during reentry are shown in Table 2 . For the burst case, the maximum discharge coefficient is used. This corresponds to the pressure dependent discharge coefficient since crossflow reduces the orifice efficiency. The XF1 discharge coefficient is used for the crush case. Similar to ascent, the pressure coefficient used to calculate the wall pressure is the minimum over all roll angles for the burst case and maximum for the crush case. The isothermal assumption is used for the crush case whereas the adiabatic assumption is used for the burst case.

Table 2. Run matrix for reentry venting analyses.

\begin{tabular}{|c|c|c|c|}
\hline & Thermal & Cd & Wall $\mathrm{cp}$ \\
\hline burst & adiabatic & Pdep & $\min \mathrm{f}(\mathrm{M}$,theta $)$ \\
\hline crush & isothermal & $\mathrm{XF1}$ & $\max \mathrm{f}(\mathrm{M}$,theta $)$ \\
\hline
\end{tabular}

\section{E. Ascent Venting Results}

The Ares I systems tunnel is vented through eight holes similar to the space shuttle solid rocket booster (SRB). Each hole is $1.13 \mathrm{in}$. in diameter and the total area of the vent holes is $8.02 \mathrm{in}^{2}$. However, each set of four vent holes is backed by a single rain shield with a minimum cross-sectional area of $2.41 \mathrm{in}^{2}$, giving a total effective vent area of $4.82 \mathrm{in}^{2}$. The holes are covered with screens, but the screens do not reduce the open area of the holes below that of the rain shields. The venting is modeled in this paper as one hole with a venting area of $4.82 \mathrm{in}^{2}$. The total compartment volume is assumed to be $19 \mathrm{ft}^{3}$ corresponding to an area/volume $(\mathrm{A} / \mathrm{V})$ ratio of $0.254 \mathrm{in}^{2} / \mathrm{ft}^{3}$.

As shown in Table 1, two runs (one for burst case and one for crush case) were performed for each of the 1,000 ascent trajectories. The burst and crush skin differential pressures for each trajectory are shown in Figure 9. The worst, 95 percentile, and 50 percentile values, as well as the trajectories corresponding to these values, are also listed. Note that, as shown in Eq. (5), the skin differential pressure is a function of time and axial location. The skin differential pressure shown here are the maximum and minimum values. For the burst case, the worst, 95 percentile, and 50 percentile skin differential pressures are 1.06, 1.04, and 1.01 psid, while those for the crush case are -2.95 , -2.70 , and -2.45 psid, respectively.

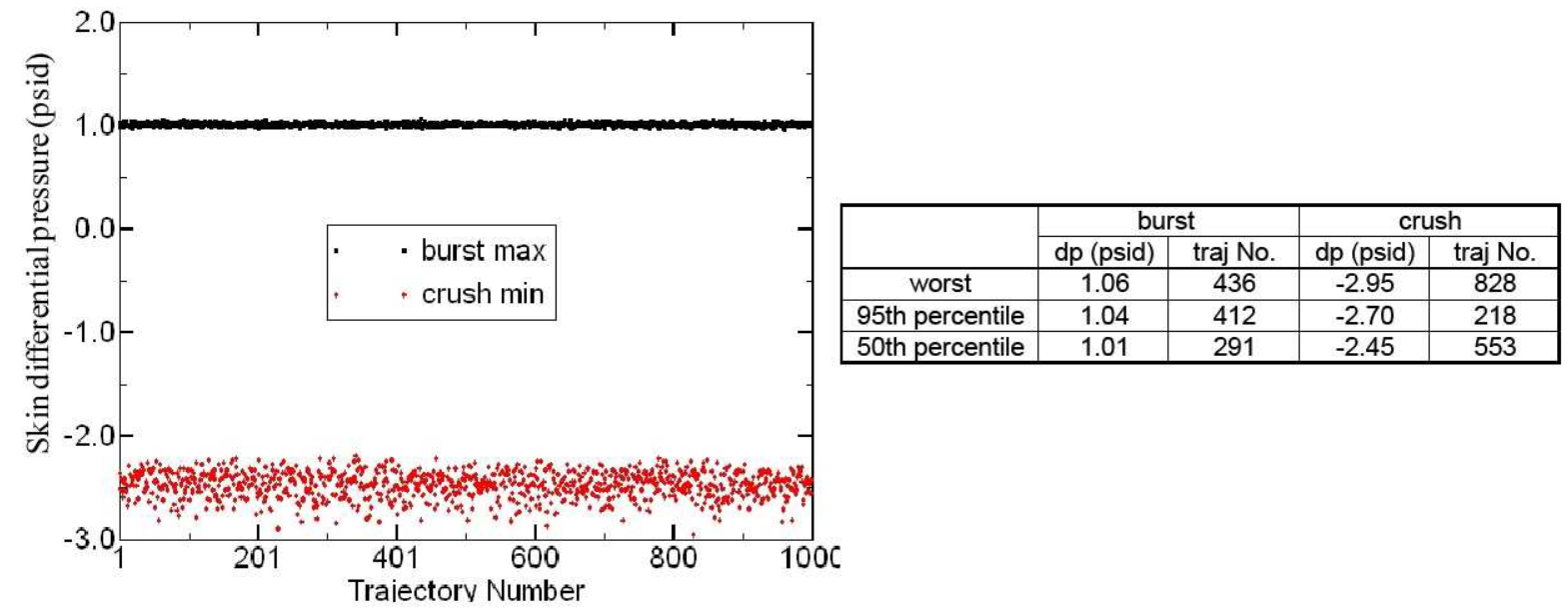

Figure 9. Predicted burst and crush skin differential pressures during ascent.

The skin differential pressure versus time for the worst, 95 percentile, and 50 percentile cases are shown in Figure 10. Both the predicted maximum and minimum skin differential pressures occur around $41 \mathrm{sec}$ where the pressure coefficient is relatively large. 


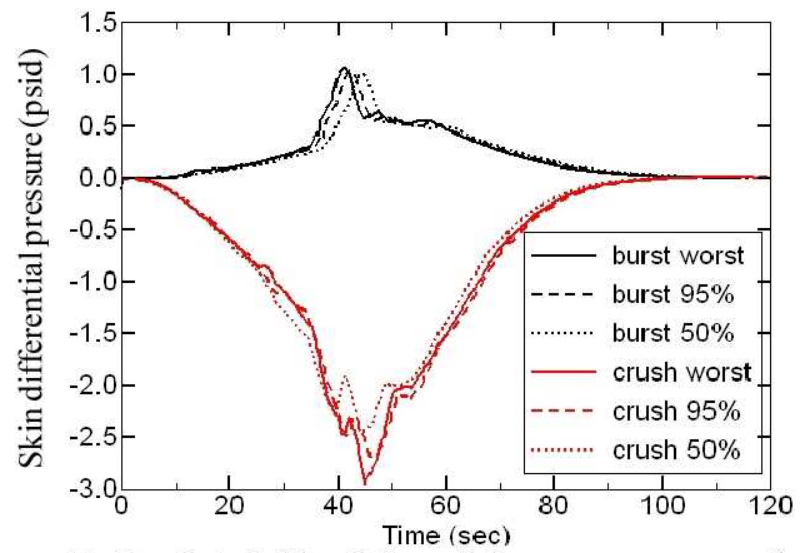

Figure 10. Predicted skin differential pressures versus time.

The predicted compartment pressure and compartment pressure relative to ambient pressure are shown in Figure 11. The compartment pressure follows the ambient pressure closely and decreases from $14.7 \mathrm{psia}$ at $0 \mathrm{sec}$ to almost zero at $100 \mathrm{sec}$. The maximum pressure difference between compartment and ambient is about $0.75 \mathrm{psid}$.
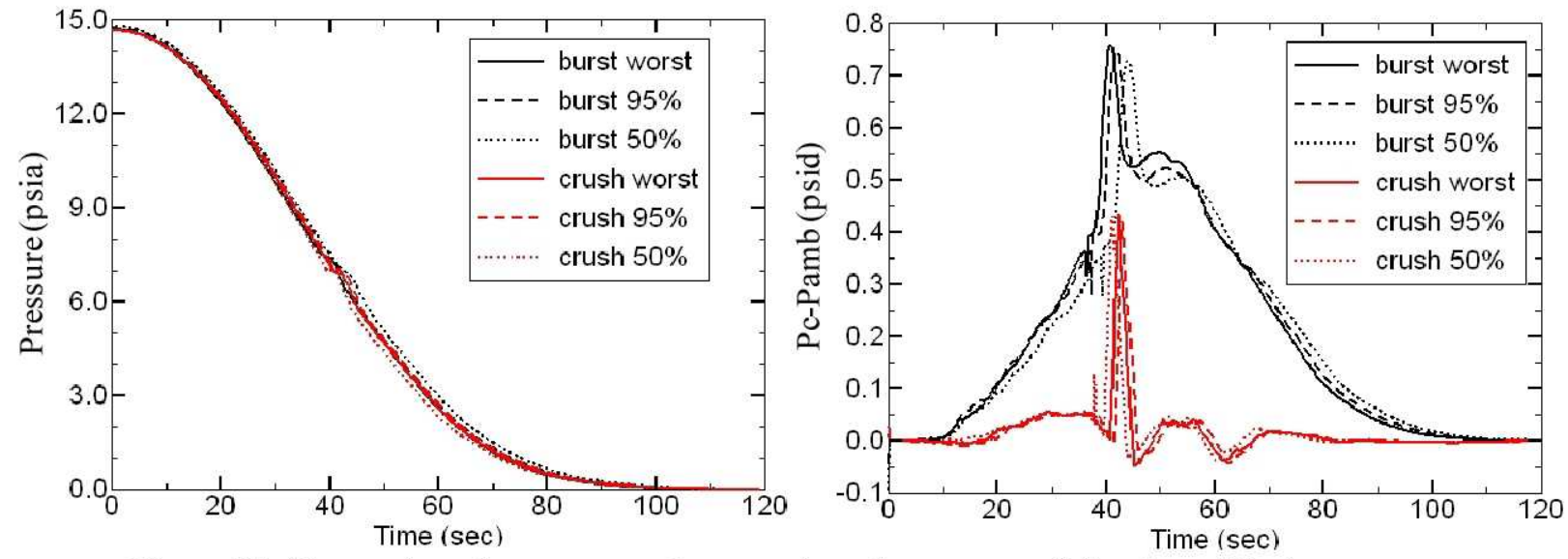

Figure 11. Compartment pressure and compartment pressure relative to ambient pressure.

Figure 12 and Figure 13 show the comparison of compartment pressure and vent local pressure as well as the ambient pressure for the burst and crush cases, respectively. The local external pressure at the vent location follows the ambient pressure closely except near $41 \mathrm{sec}$ where the pressure coefficient is large. The compartment pressure is much closer to the vent local pressure for the crush cases than the burst cases because the pressure dependent discharge coefficients applied for the crush cases are larger than the crossflow dependent discharge coefficients used for the burst cases.
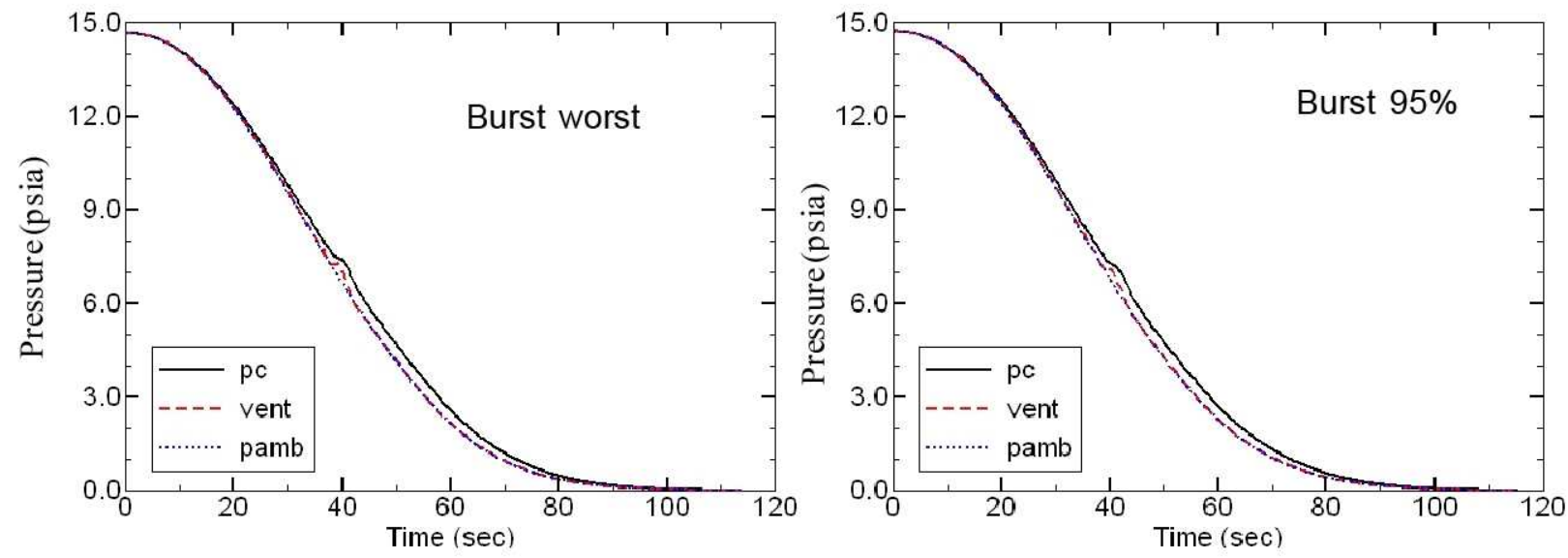

Figure 12. Comparison of predicted compartment pressure, vent local pressure, and ambient pressure for the burst cases. 

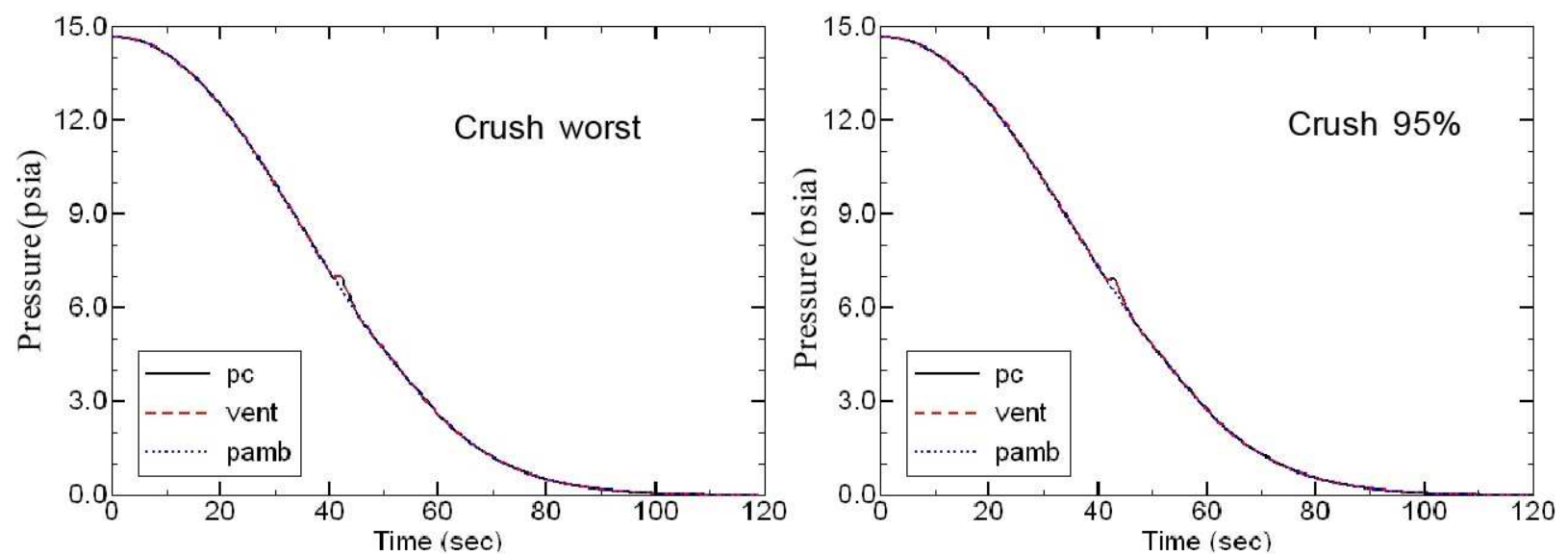

Figure 13. Comparison of predicted compartment pressure, vent local pressure, and ambient pressure for the crush cases.

\section{F. Reentry Venting Results}

As shown in Figure 3, the ambient pressure before $320 \mathrm{sec}$ for the mean reentry trajectory is less than 1 psfa. Thus, the reentry venting analyses start from the time when the ambient pressure is about 1 psfa and assume the pressure and temperature inside the systems tunnel are the same as the ambient conditions at this time. As shown in Table 2, two runs (one for burst case and one for crush case) are performed for each of the 1,000 reentry trajectories. The burst and crush skin differential pressures for each trajectory are shown in Figure 14. The worst, 95 percentile, and 50 percentile values as well as the trajectories corresponding to these values are also listed. The skin differential pressures during reentry have a much larger range compared to ascent due to the large range in dynamic pressures and pitch angles (see Figure 4). For the burst case, the worst, 95 percentile, and 50 percentile skin differential pressures are $10.68,9.37$, and 6.93 psid, while those for the crush case are $-23.72,-14.29$, and $-10.66 \mathrm{psid}$, respectively.

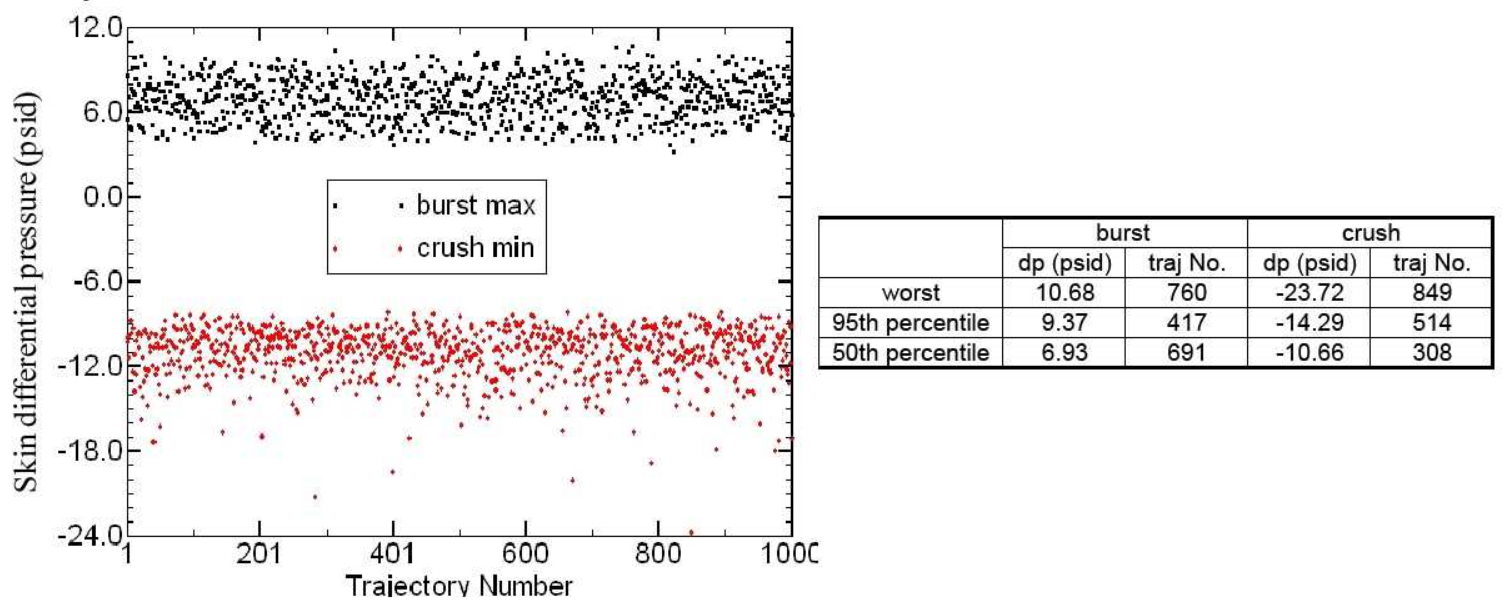

Figure 14. Predicted burst and crush skin differential pressures during reentry.

The skin differential pressure versus time for the worst, 95 percentile,, and 50 percentile cases are shown in Figure 15. The predicted maximum skin differential pressure occurs near $388 \mathrm{sec}$ whereas the minimum occurs near $362 \mathrm{sec}$. The oscillations in pressures shown here and in the next few figures are caused by the oscillations in pitch and roll angles as well as the relatively large area/volume ratio of the systems tunnel venting configuration, which allows the pressure inside the systems tunnel to follow the vent local pressure closely. 


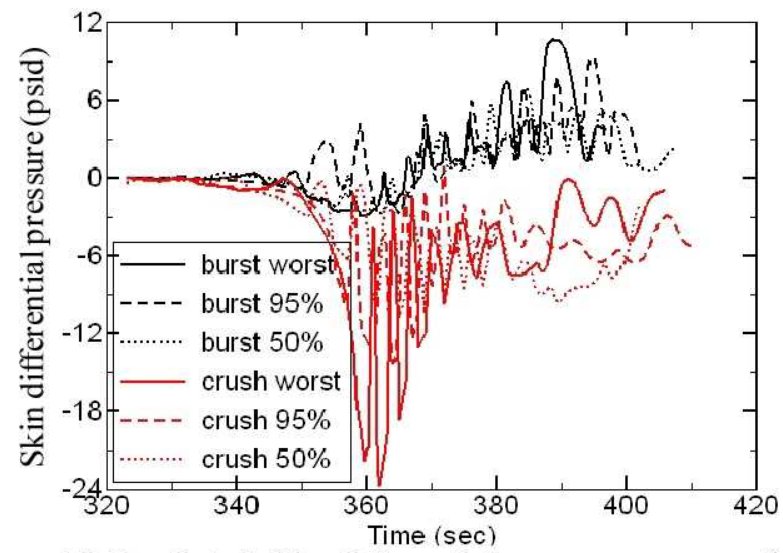

Figure 15. Predicted skin differential pressures versus time.

The predicted compartment pressure and compartment pressure relative to ambient pressure are shown in Figure 16. The maximum pressure difference between compartment and ambient is about 6.5 psid and occurs near $358 \mathrm{sec}$.
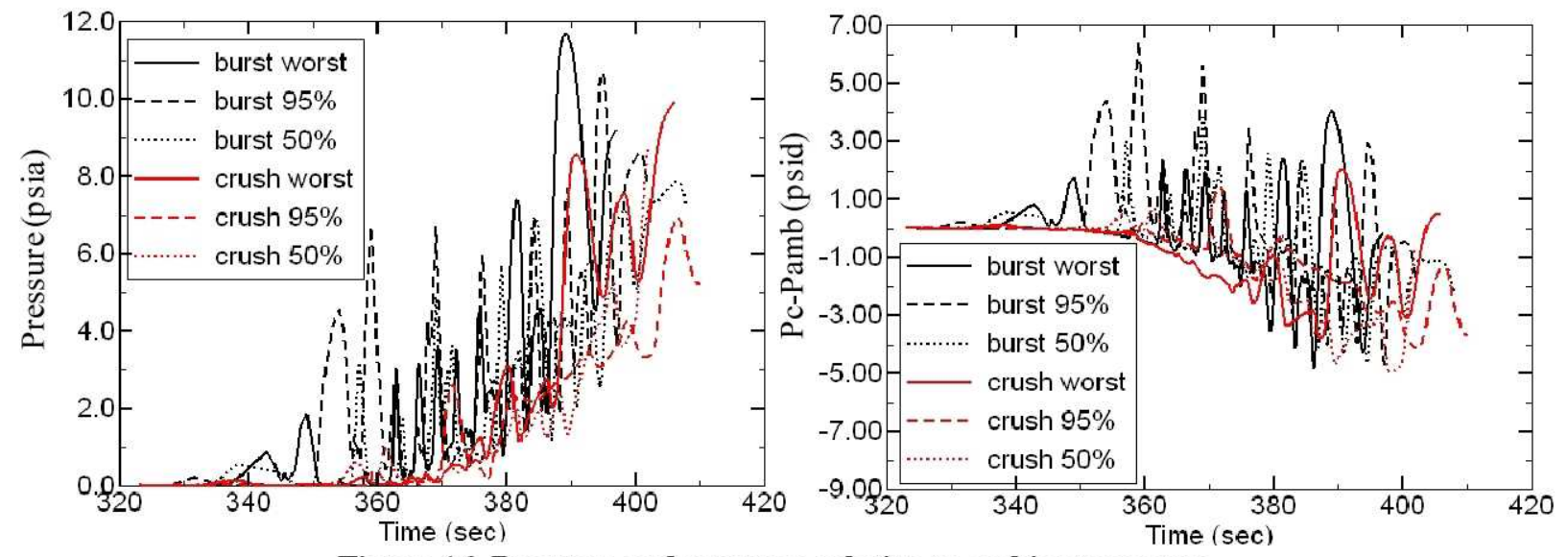

Figure 16. Pressure and pressure relative to ambient pressure.

Figure 17 and Figure 18 show the comparison of compartment pressure and vent local pressure, as well as the ambient pressure for the burst and crush cases, respectively. The local external pressure at the vent location does not follow the ambient pressure closely due to the large pitch and roll angles. The predicted compartment pressure oscillates because of the large area/volume ratio. The compartment pressure is much closer to the vent local pressure for the burst cases than the crush cases because the pressure dependent discharge coefficients applied for the burst cases are larger than the crossflow dependent discharge coefficients used for the crush cases.
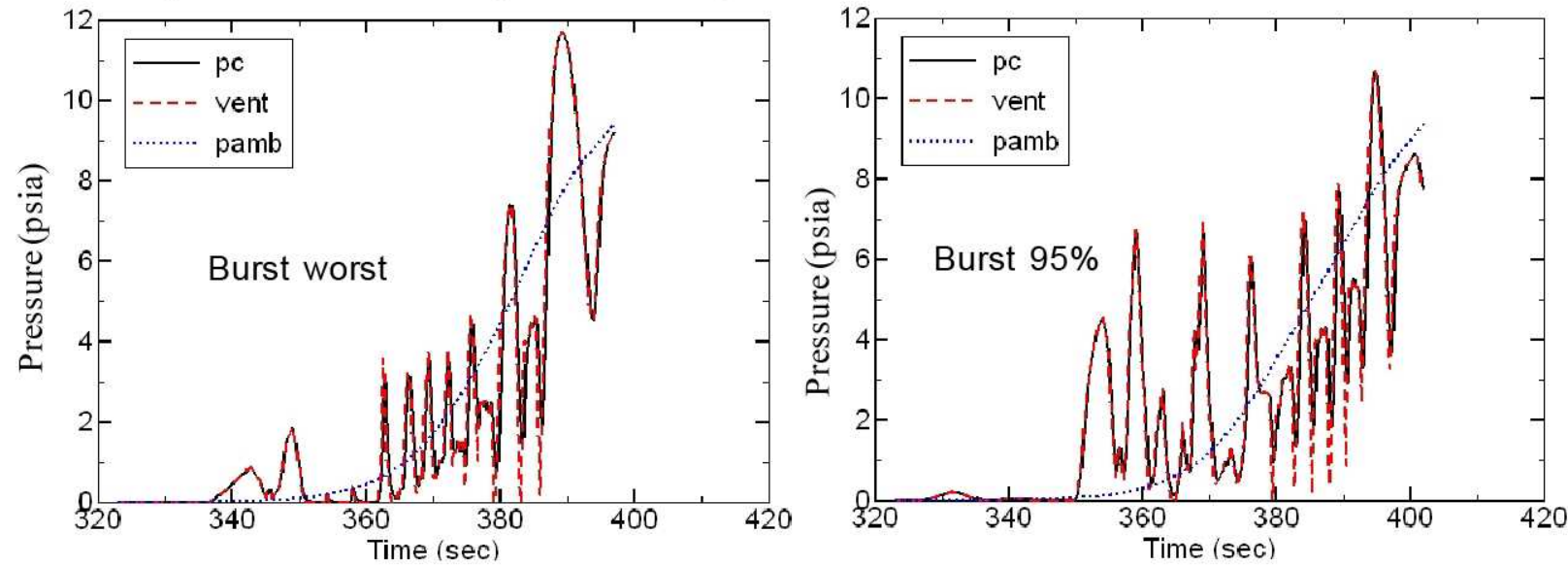

Figure 17. Comparison of predicted compartment pressure, vent local pressure, and ambient pressure for the burst cases. 

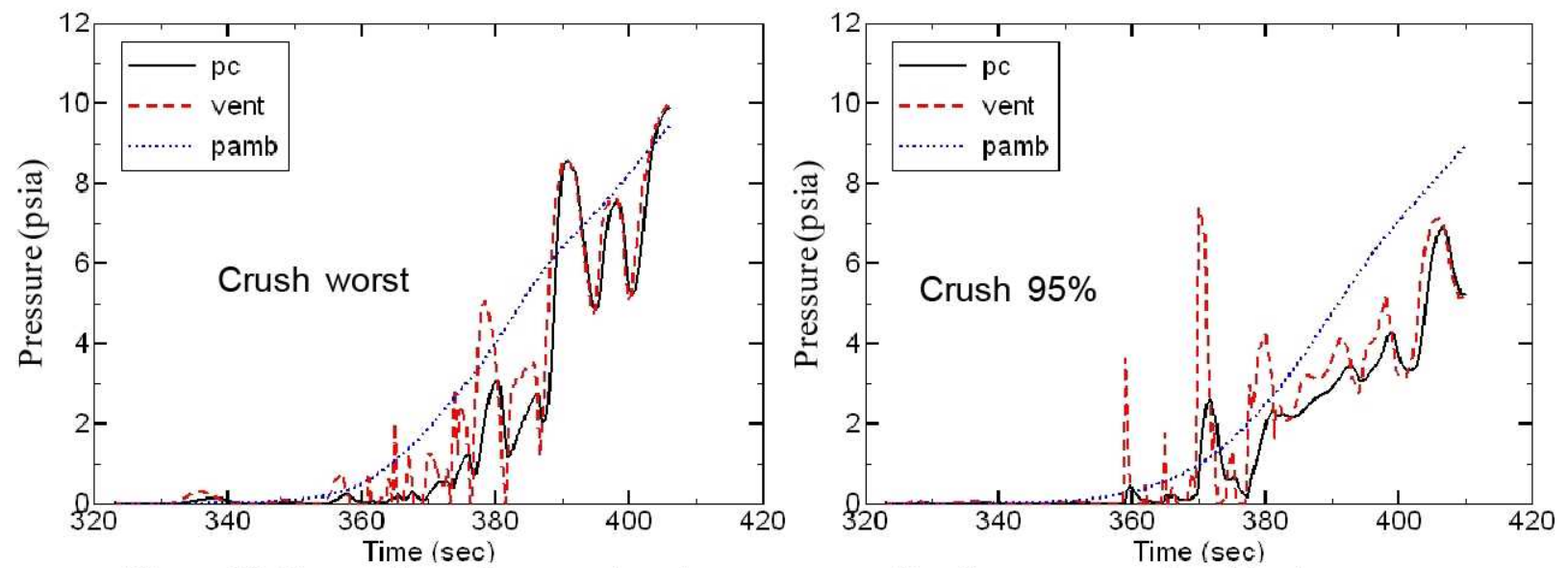

Figure 18. Comparison of compartment pressure, vent local pressure, and ambient pressure for the crush cases.

\section{3D Transient CFD Analyses}

Results shown in the last section treat the systems tunnel as a single compartment for the entire length of 1,396 inches (i.e., pressure differential inside the systems tunnel is neglected). In Ref. 3, the Ares I upper stage systems tunnel was divided into 20 small chambers and the internal gas was allowed to flow from one chamber to the next. The orifice area that allows flow between the chambers was assumed to be $1 \mathrm{in}^{2}$ and each chamber was assumed to be individually vented to the outside through a vent area of $1.3 \mathrm{in}^{2}$. These areas; however, seem to be quite arbitrary. In order to determine the pressure and temperature differences inside the systems tunnel, 3D transient CFD venting analyses were performed and results are documented in this section.

The commercial CFD code FLUENT is used for all CFD runs shown in this paper. Both pressure-based and density-based solvers in FLUENT are applied to the venting problem. It was found that the density-based solver takes much longer to run (the computer processing unit [CPU] time for each time step is much larger and a smaller time step has to be applied) but results from these two solvers are very similar. Therefore, only results from the pressure-based solver are shown in this paper. The flow is assumed to be laminar since the gas velocity inside the tunnel is very small although the ambient Mach number is very large during later ascent and early reentry.

Although not shown here, the predicted pressures inside the systems tunnel using different time steps were compared and it was found that a time step of $1 \mathrm{~ms}$ is appropriate for the CFD simulations during both ascent and reentry. This time step is reduced to $0.1 \mathrm{~ms}$ for runs with high pressure rising rates shown in Section III.C.2 and large pitch angles in Section III.C.3. Furthermore, much smaller time steps are used at the beginning of all reentry CFD calculations. Specifically, 100 time steps are run using time steps of $0.01 \bigotimes_{s}, 0.1 \bigotimes_{s}, 1 \bigotimes_{s}, 0.01 \mathrm{~ms}$, and 0.1 $\mathrm{ms}$ before the time step is increased to $1 \mathrm{~ms}$.

All CFD results shown in this paper were performed on a Linux cluster with AMD Opteron $2.6 \mathrm{GHz}$ processors. To run for $120 \mathrm{sec}$ real time, the wall clock time is about 12 months using one CPU and 1.1 months using 32 CPUs. It was found that using more than $32 \mathrm{CPUs}$ does not reduce the wall clock time much. Note that these times are much larger than the time it takes to run CHCHVENT, which is only a few minutes for each trajectory.

While the CHCHVENT predictions used 1,000 trajectories during both ascent and reentry as discussed in Section II, only the mean trajectories shown in Figure 2 and Figure 3 are used in the CFD simulations due to the long simulation time required. The pitch angles in the CFD runs are assumed to be zero for both ascent and reentry.

\section{A. Geometry and Grid}

Figure 19 shows the flight cable layout and the cross-section geometry in the y-z plane used in the CFD model. The $\mathrm{x}$ axis is in the axial direction. Note that the individual cables are not modeled and it is assumed that the cross section area is the same at different axial locations. 
Flight Cable Layout

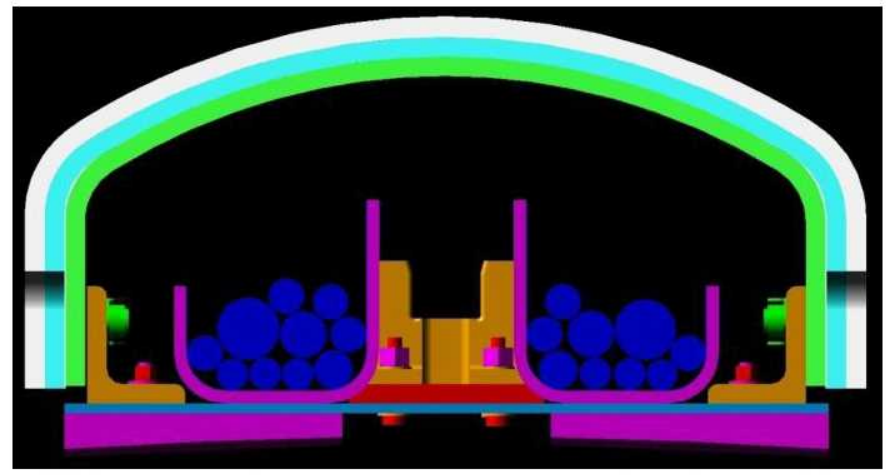

CFD Model

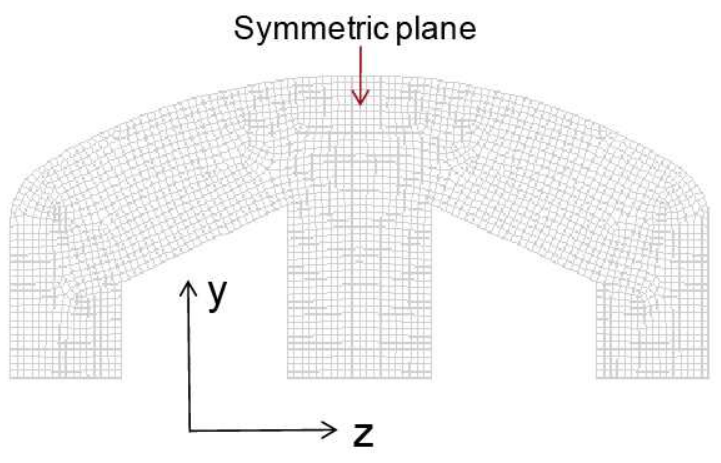

Figure 19. Cross-section geometry in the y-z plane.

The geometry of the systems tunnel CFD model is shown in Figure 20. As discussed in Section II, there are eight venting holes, two rain shields, and four screens. In the 3D CFD simulations, the rain shields and screens are not modeled and the eight vents are modeled as one hole with an area equal to the minimum venting area due to the rain shield.

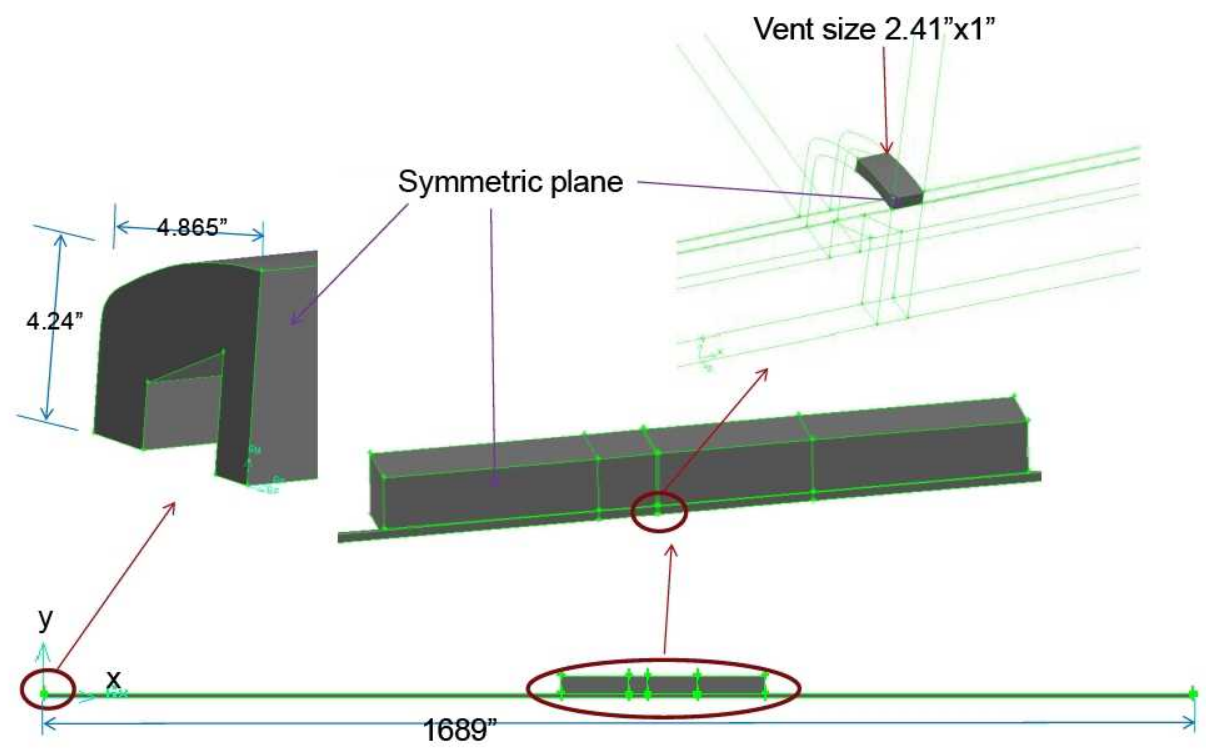

Figure 20. Geometry of the systems tunnel.

Figure 21 shows the grid in the $x-y$ symmetric plane and $y-z$ plane at the center of the vent. The computational domain includes the total length of the systems tunnel and the vent, as well as the area outside the vent. That is, both the internal flow inside the tunnel and external flow outside the tunnel are simulated. 


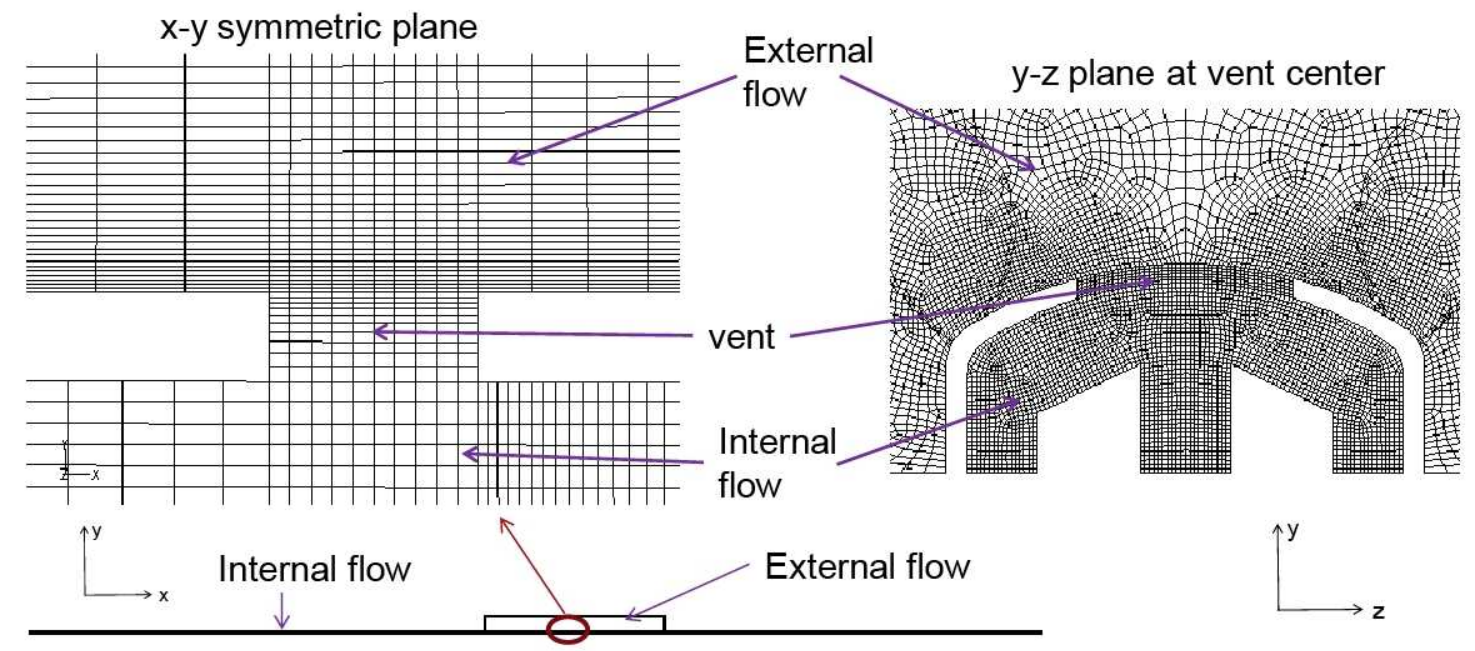

Figure 21. Geometry and grid in the $x$-y symmetric plane at the vent center.

The surface grid is shown in Figure 22. Due to symmetry, only half of the y-z plane is modeled. Note that, while the whole length of the systems tunnel is modeled, other parts of the Ares I vehicle such as first stage solid rocket motor and upper stage are not included in the CFD model. There are about 1.32 million hexahedral flow cells.

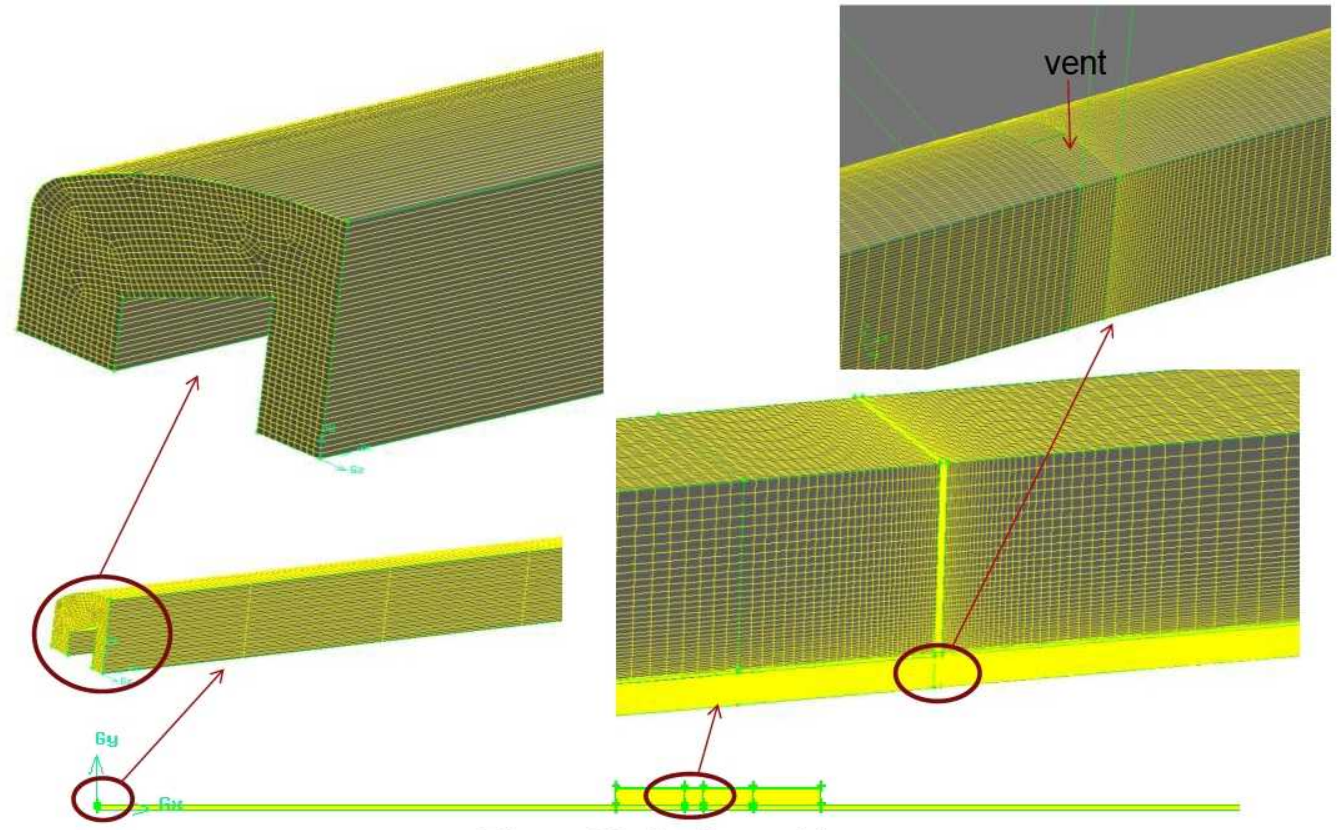

Figure 22. Surface grid.

\section{B. Ascent Venting Results}

Figure 23 shows the predicted maximum, average, and minimum pressures inside the systems tunnel as well as the differences between these pressures during ascent. As expected, the predicted pressures inside the tunnel are larger than the ambient pressure. It is evident that the pressures at different locations inside the systems tunnel are very similar. Specifically, the difference between maximum and minimum pressure is smaller than 0.01 psid, indicating that the uniform pressure assumption is valid. 

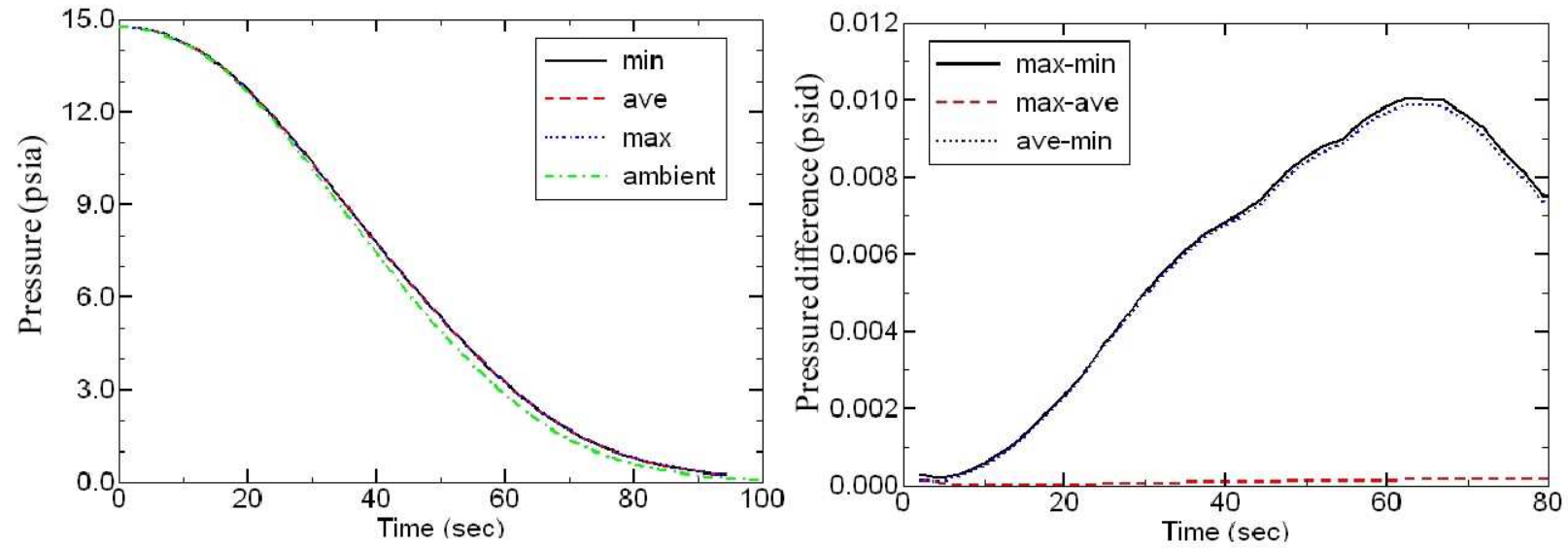

Figure 23. Predicted maximum, average, and minimum pressures inside the systems tunnel and their differences.

Pressures at all cells inside the tunnel at $59.5 \mathrm{sec}$ are shown in Figure 24. Consistent with Figure 23, the largest difference between maximum and minimum pressure is about $0.01 \mathrm{psid}$, which occurs near the vent location. Away from the vent, the difference between maximum and minimum pressure is only about $0.0002 \mathrm{psid}$.
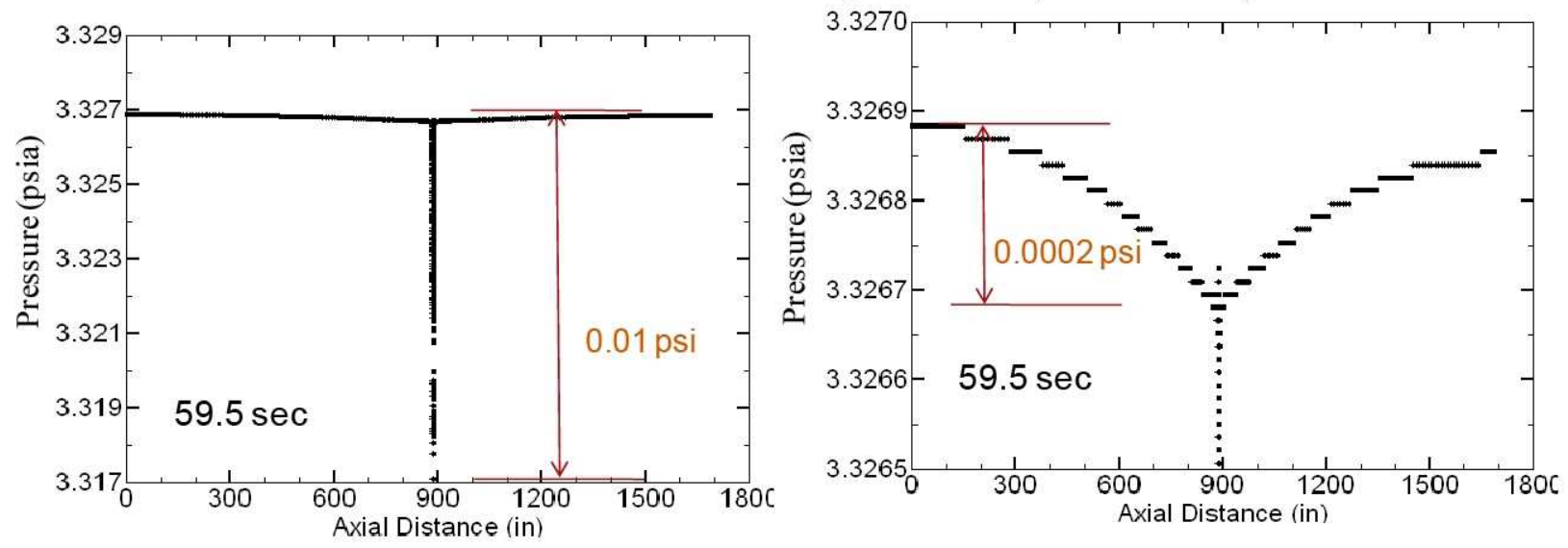

Figure 24. Pressure at all cells inside the tunnel at $59.5 \mathrm{sec}$.

The predicted maximum, average, and minimum temperatures inside the systems tunnel are shown in Figure 25 together with the differences between them. The gas temperature inside the tunnel is smaller than the ambient temperature. Similar to pressure, the difference between maximum and minimum temperature is smaller than $0.3{ }^{\circ} \mathrm{R}$, indicating that the uniform temperature assumption inside the systems tunnel is also valid during ascent.
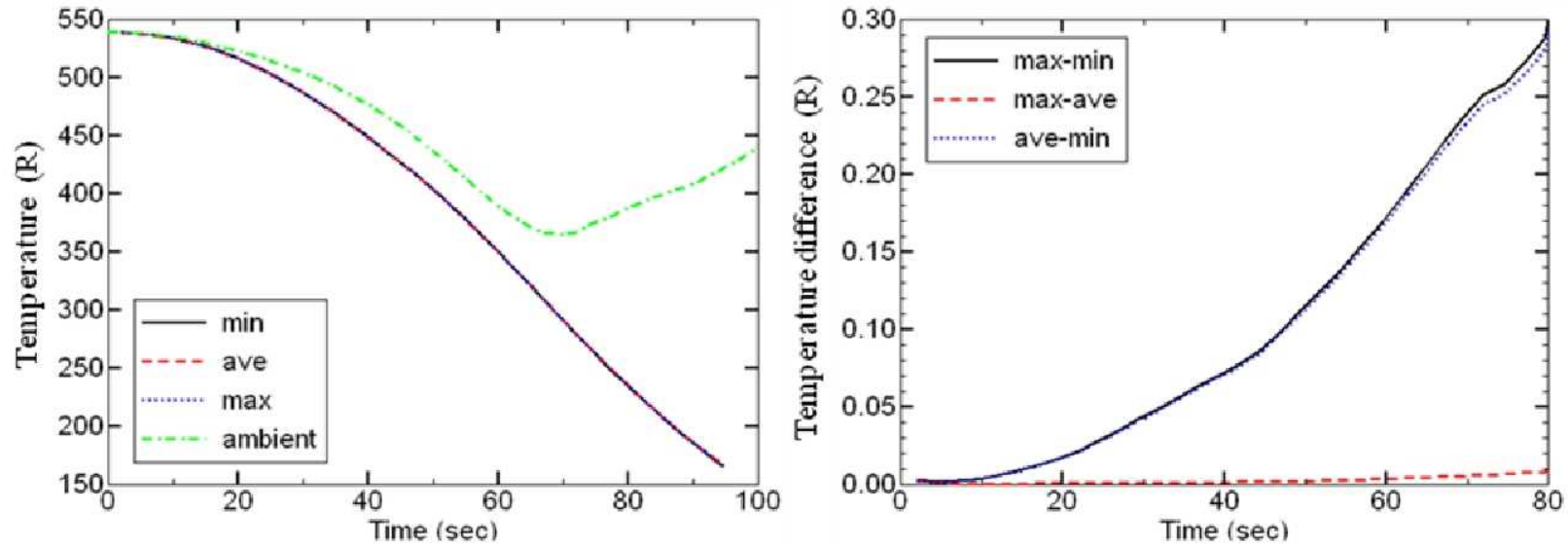

Figure 25. Predicted maximum, average, and minimum temperatures inside the systems tunnel and their differences. 
Pressure contours in the $x-y$ symmetric plane and the $y-z$ plane at the vent center are shown in Figure 26 and Figure 27, respectively. As expected, the pressure is higher inside the tunnel than outside. The shock wave in the external flow near the vent is also observed. The angle between the shock wave and axial direction decreases with time due to the increase in freestream Mach number.

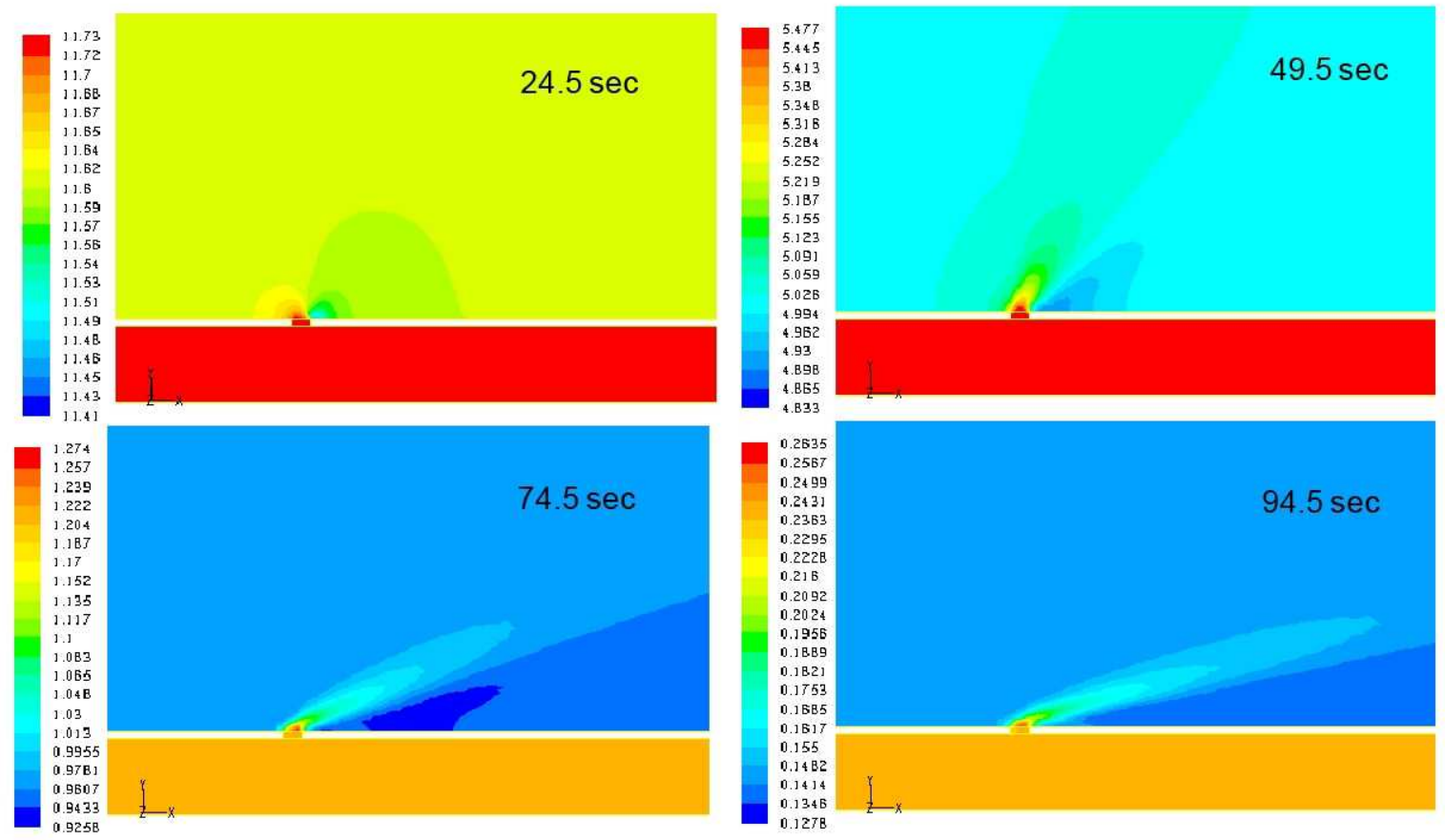

Figure 26. Pressure (psia) contours in the symmetric plane.

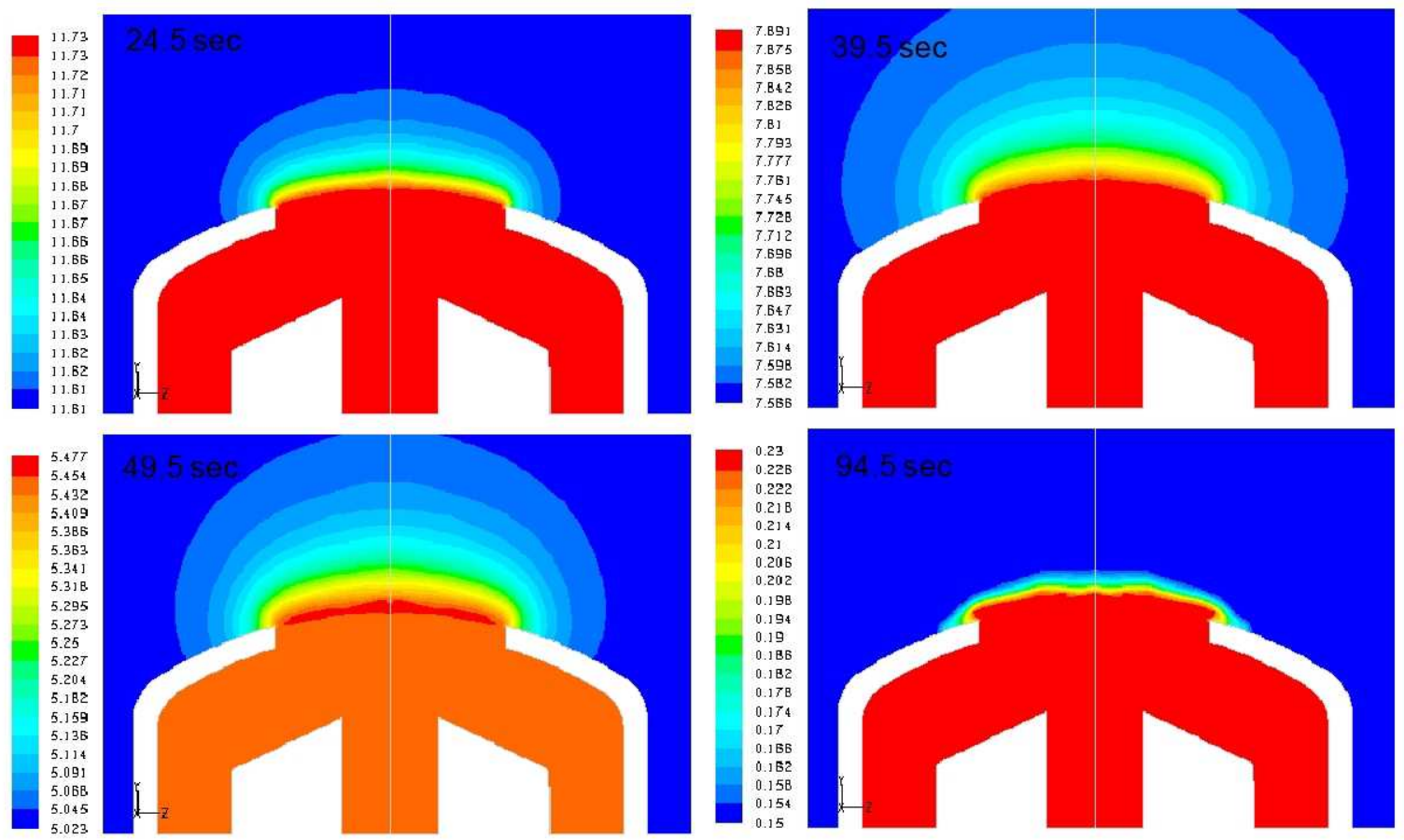

Figure 27. Pressure (psia) contours in the y-z plane.

Figure 28 and Figure 29 show the velocity vectors colored by Mach number and the streamlines near the vent at the symmetric plane, respectively. Only velocity components in the $\mathrm{x}$ and $\mathrm{y}$ directions are used to calculate the streamlines. As expected, the flow is from the systems tunnel to the outside through the vent. 


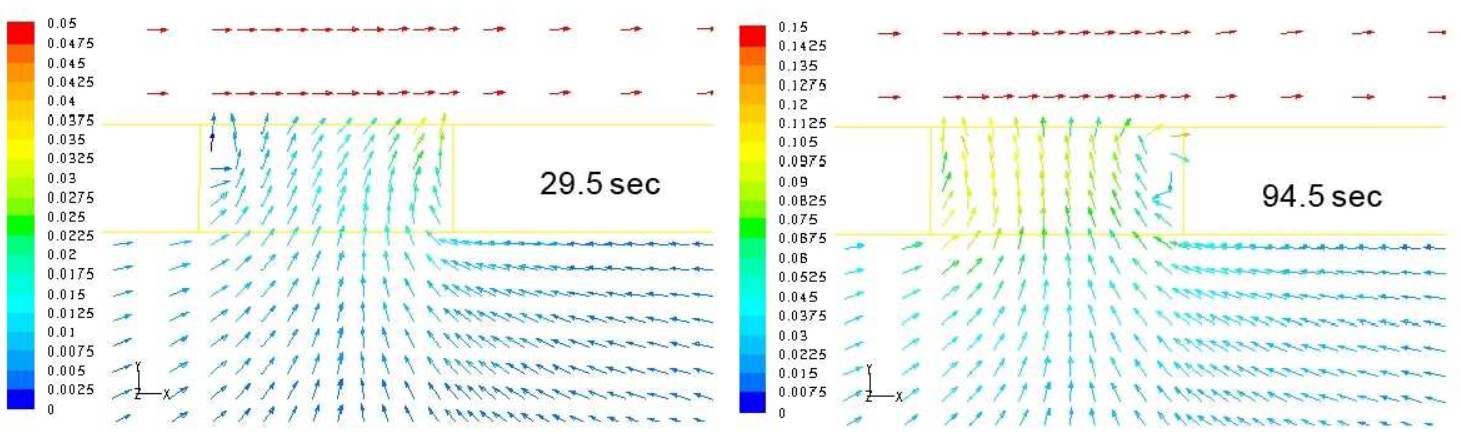

Figure 28. Velocity vectors colored by Mach number near the vent at the symmetric plane.
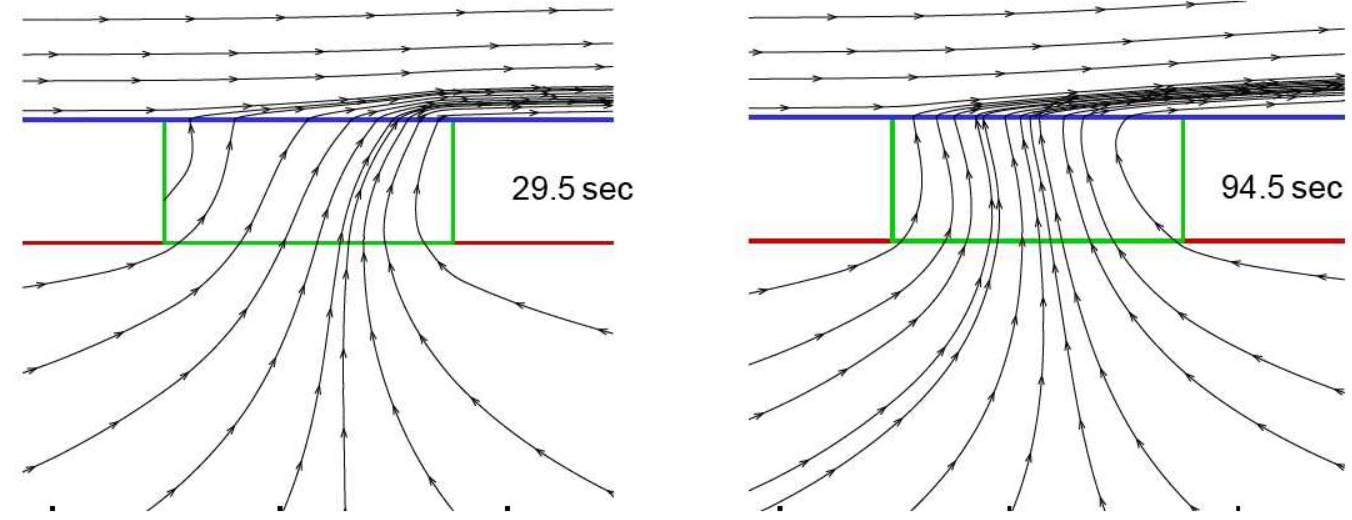

Figure 29. Streamlines near the vent in the symmetric plane.

\section{Reentry Venting Results}

As shown in Figure 3, the ambient pressure before $354 \mathrm{sec}$ during reentry is very small. Thus, the reentry CFD analyses start from $354 \mathrm{sec}$ and assume the pressure and temperature inside the systems tunnel are the same as the ambient conditions whereas the gas velocity everywhere inside the tunnel is zero at this time. Results from the baseline run are discussed first followed by those using high pressure rising rates, large pitch angles, and isothermal wall boundary conditions.

\section{Baseline Results}

The predicted maximum, average, and minimum pressures inside the systems tunnel as well as the differences between them are shown in Figure 30. As expected, the predicted average pressure inside the tunnel is smaller than the ambient pressure. The difference between maximum and minimum pressure is smaller than 0.67 psid during reentry indicating that the uniform pressure assumption inside the systems tunnel is reasonable, although this difference is much larger than during ascent.
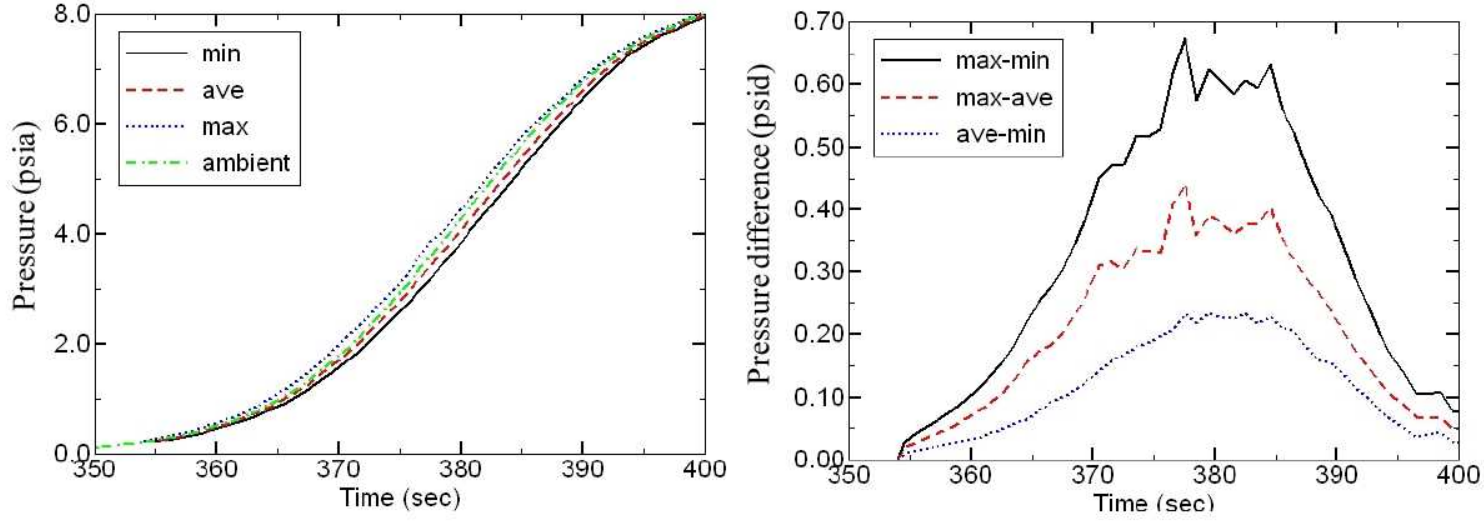

Figure 30. Predicted maximum, average, and minimum pressures and their differences. 
Figure 31 shows the predicted pressures at all cells inside the tunnel at $376.5 \mathrm{sec}$ when the pressure difference is the largest. Consistent with Figure 30, the difference between maximum and minimum pressure is about 0.62 psid. However, both minimum and maximum pressures occur near the vent location. Away from the vent, the difference between maximum and minimum pressure is only about $0.0013 \mathrm{psid}$.
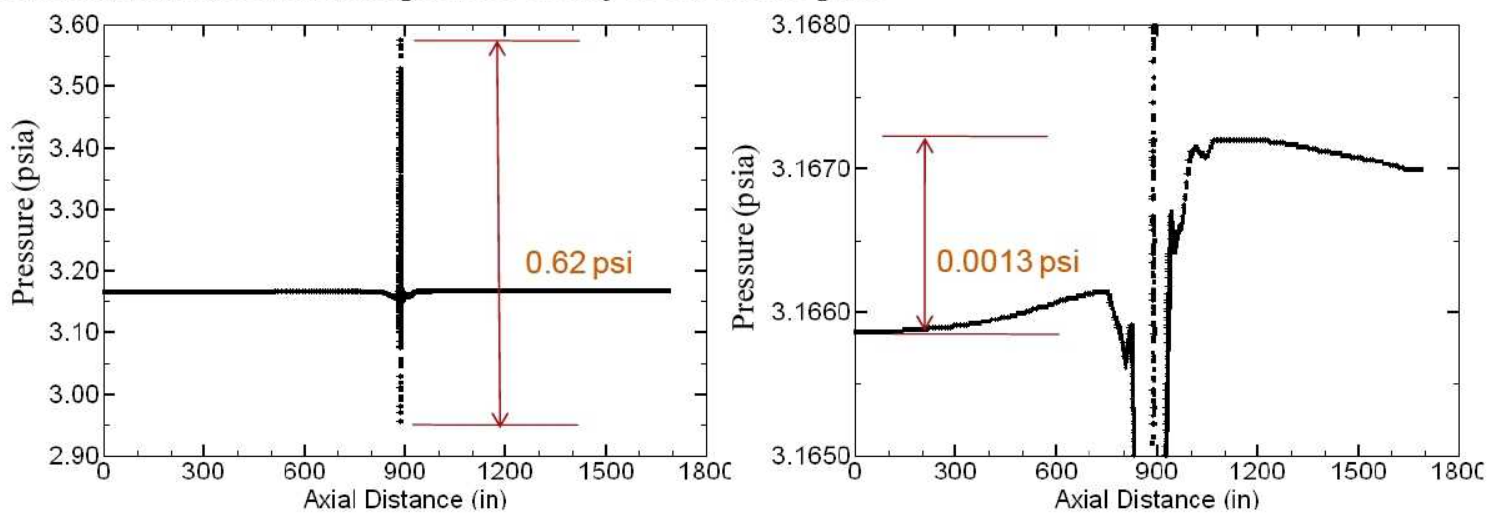

Figure 31. Pressure at all cells inside the tunnel at $376.5 \mathrm{sec}$.

The predicted maximum, average, and minimum temperatures inside the systems tunnel are shown in Figure 32 together with the differences between them. As expected, even the minimum gas temperature inside the tunnel is larger than the ambient temperature due to the conversion of kinetic energy to thermal energy. In contrast to the pressure; however, the difference between maximum and minimum temperature is as much as $3,150{ }^{\circ} \mathrm{R}$ indicating that the uniform temperature assumption is not valid during reentry.
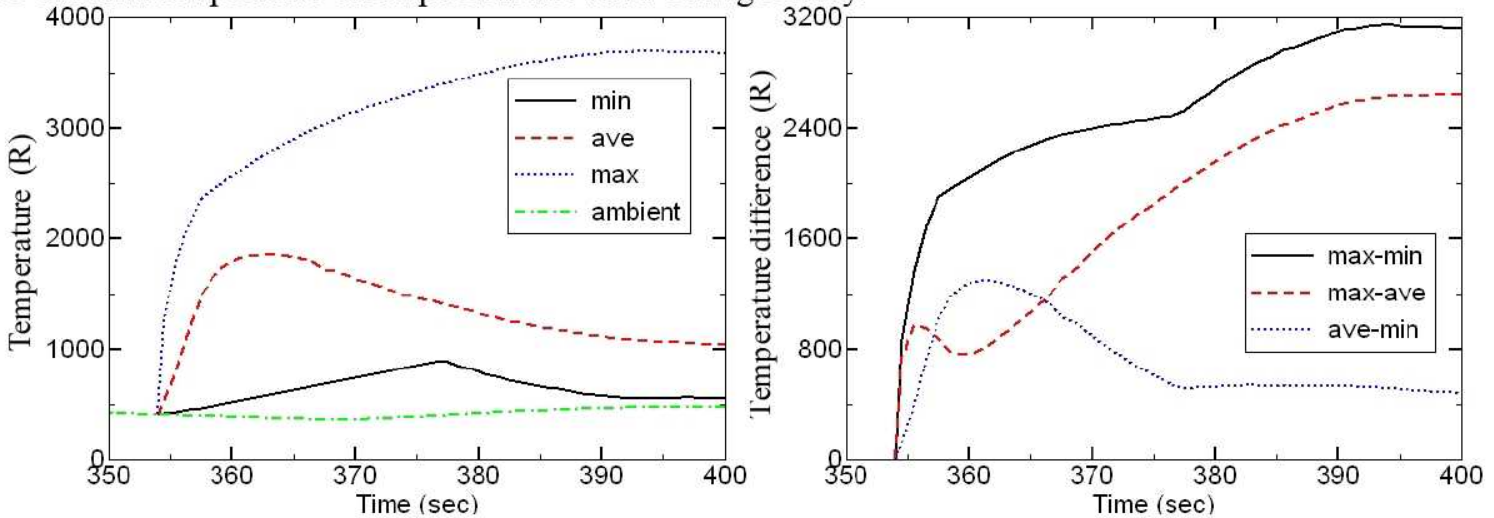

Figure 32. Predicted maximum, average, and minimum temperatures and their differences.

Velocity vectors and streamlines near the vent in the symmetric plane at $356.5 \mathrm{sec}$ are shown in Figure 33. There is only a very small amount of gas flowing from ambient to the vent. Near the left side of the vent, the flow is from the systems tunnel to the vent whereas it is from the vent to the tunnel near the right side. Although not shown here, the streamlines and velocity vectors are similar at other times.

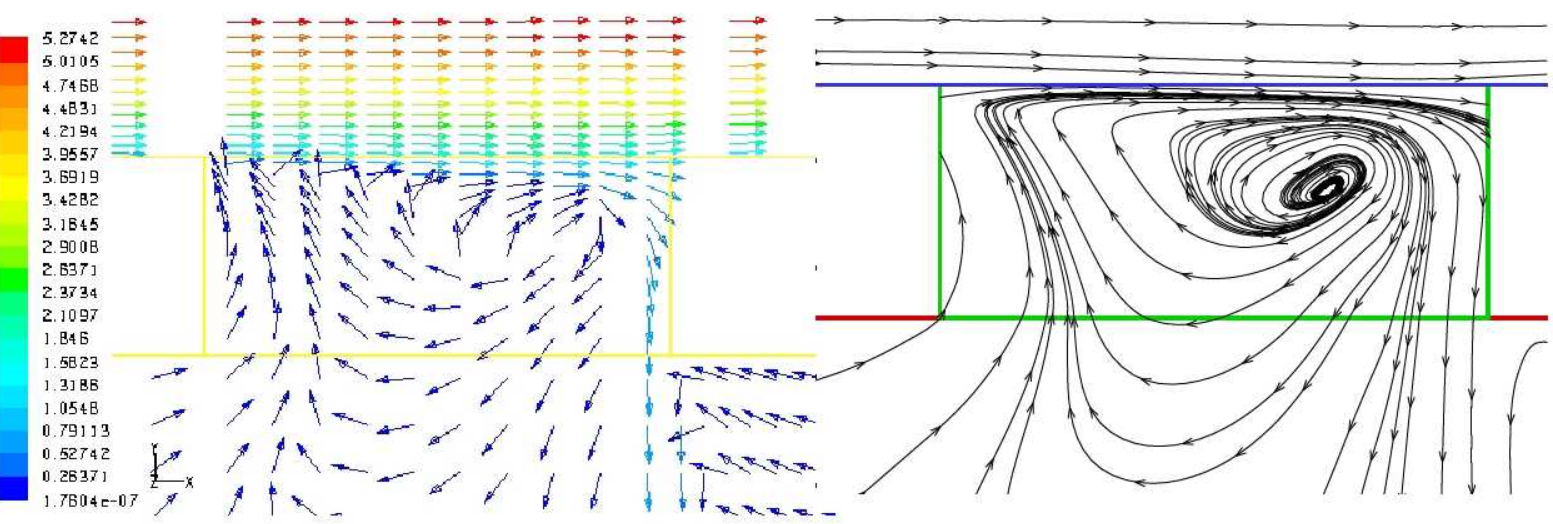

Figure 33. Streamlines and velocity vectors near the vent at the symmetric plane at 356.5 sec. 
The flow patterns in Figure 33 show that there is not much gas flowing from outside into the tunnel. This is because, as shown in Figure 34, the average pressure inside the tunnel is very close to the ambient pressure. According to Eqs. (1) and (2), the flow Mach number and mass flow rate approach zero when the compartment pressure approaches the ambient pressure. Furthermore, as shown in Figure 7 and Figure 8, the discharge coefficient is also small when the pressure ratio is near unity. In fact, using the pressure ratio in Figure 34 and the freestream Mach number in Figure 3, the discharge coefficients can be calculated from Figure 8 as 0.013 , which is much smaller than unity.

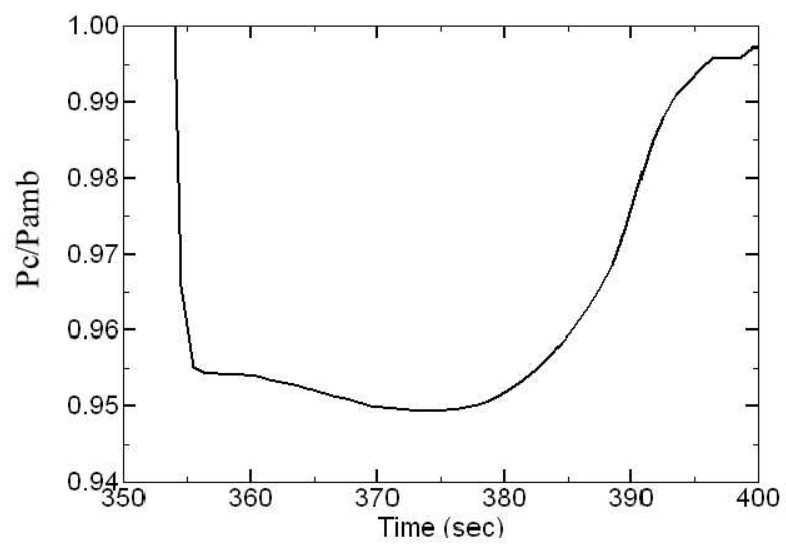

Figure 34. Ratio of pressure inside the tunnel and ambient pressure.

\section{Effects of Pressure Rising Rates}

As discussed above, there is not much gas flowing from outside into the tunnel due to the fact that the pressure ratio as shown in Figure 34 is close to unity. Figure 35 shows the ratio of pressure inside the tunnel and ambient pressure when the pressure rising rate is increased from $0.033 \mathrm{psid} / \mathrm{sec}$ to $2.26 \mathrm{psid} / \mathrm{sec}$ and $25.3 \mathrm{psid} / \mathrm{sec}$. For both of the high pressure rising rates, the pressure ratio is about 0.65 at $355 \mathrm{sec}$.

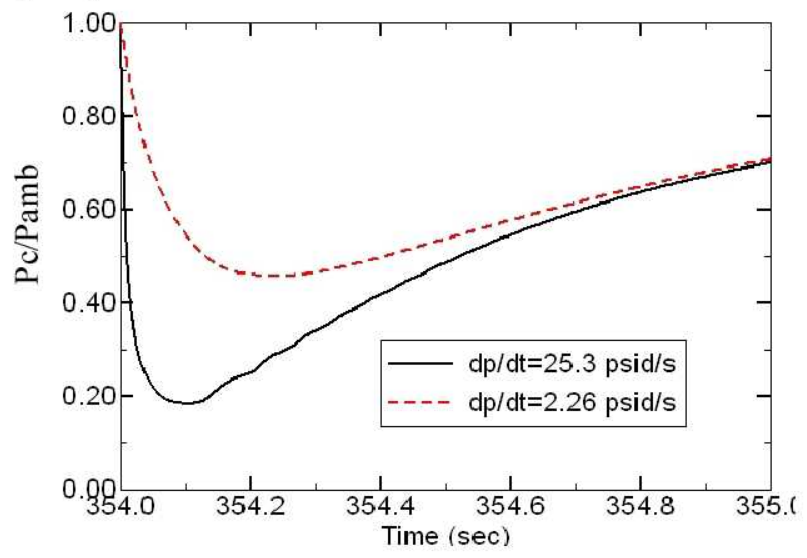

Figure 35. Ratio of pressure inside the tunnel and ambient pressure for higher pressure rising rate cases.

Velocity vectors and streamlines near the vent in the symmetric plane for the higher pressure rising rate case are shown in Figure 36 and Figure 37, respectively. Using the pressure ratio in Figure 35 and the Mach number in Figure 3, the discharge coefficients can be calculated from Figure 8 as 0.34 and 0.086 at 354.16 and $355.0 \mathrm{sec}$, respectively. As expected, the larger discharge coefficient corresponds to more gas flowing into the systems tunnel from outside. 

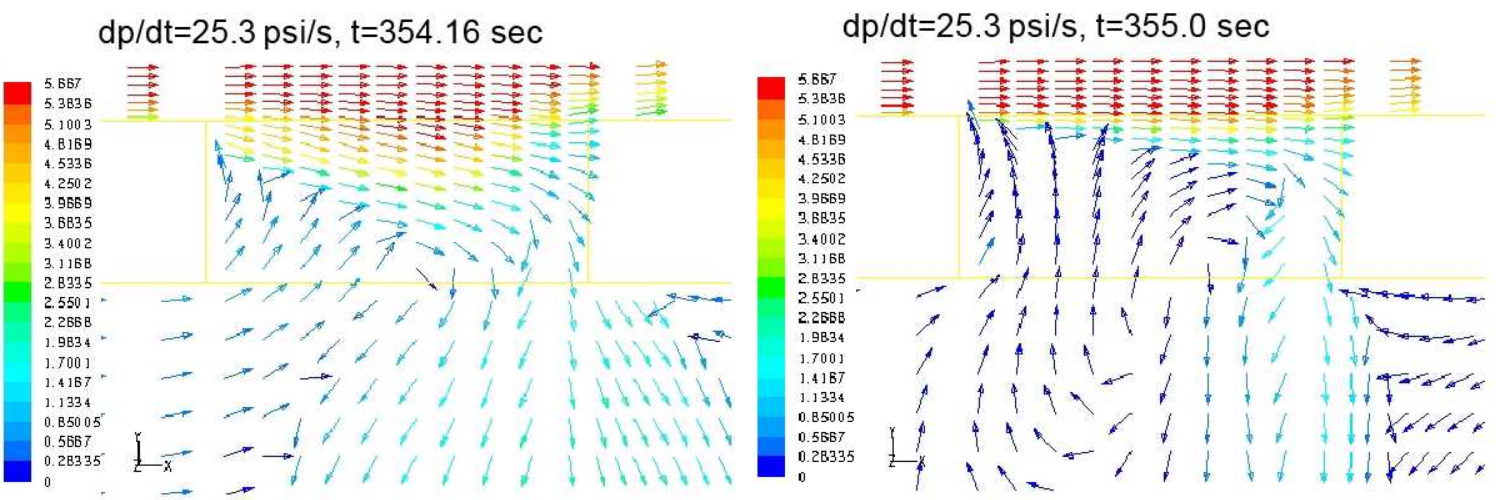

Figure 36. Velocity vectors colored by Mach number for the higher pressure rising rate case.

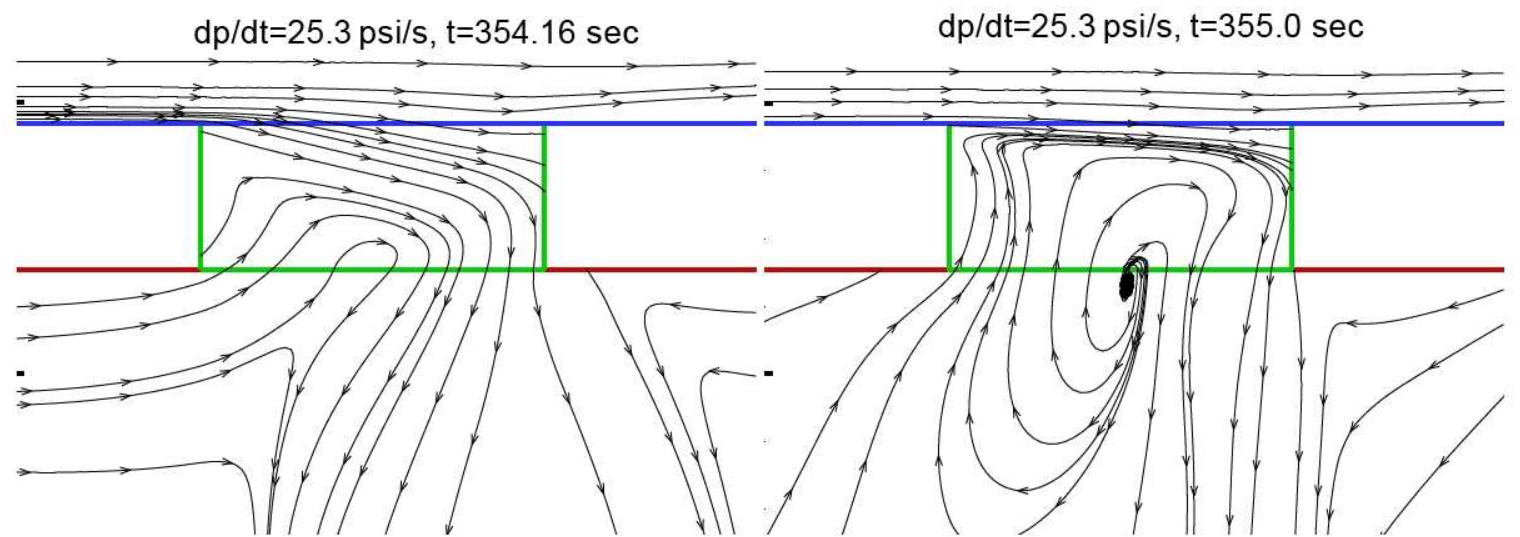

Figure 37. Streamlines near the vent for the higher pressure rising rate case.

\section{Effects of Pitch Angles}

All results shown above are for a pitch angle of 0 degrees, which means the ambient flow is in the axial direction. Figure 38 shows streamlines near the vent in the symmetric plane for pitch angles of 45 and 90 degrees. As expected, the flow is from ambient to the tunnel through the vent for these high pitch angels. In contrast to those shown in Figure 33, there is no flow from the tunnel to the vent.

Pitch angle $=45^{\circ}$

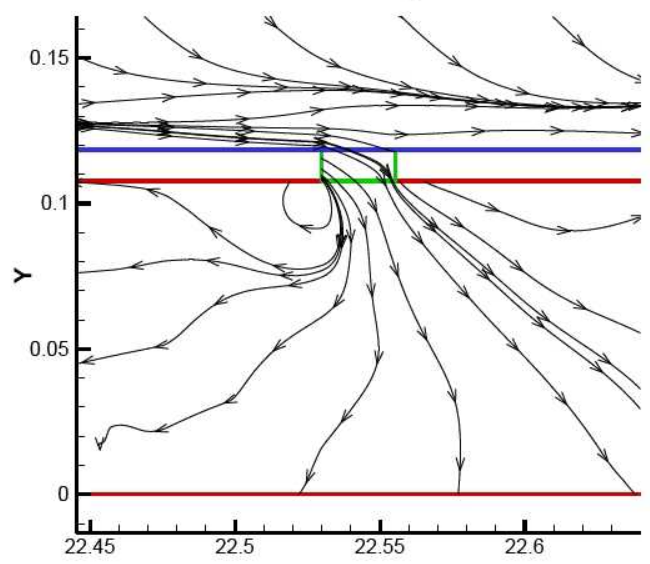

Pitch angle $=90^{\circ}$

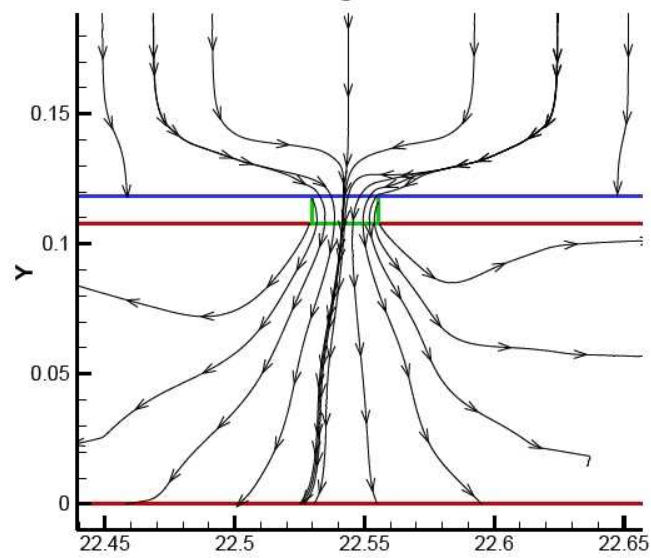

Figure 38. Streamlines at the symmetric plane near the vent for pitch angels of 45 and 90 degrees.

\section{Effects of Wall Boundary Conditions}

The predicted gas temperature inside the systems tunnel shown in Figure 32 is as high as $3,700{ }^{\circ} \mathrm{R}$, which would melt everything inside the tunnel. This predicted high temperature is due to the fact that the adiabatic wall boundary 
condition was applied and the heat transfer between gas and solid was neglected. To study the effect of wall boundary conditions, another CFD run was performed by assuming isothermal wall boundary conditions at a constant wall temperature of $300 \mathrm{~K}$. Note that the isothermal boundary condition used in CFD, where the wall temperature is kept constant, is different from the isothermal assumption in CHCHVENT discussed in Section II, where the gas temperature is assumed to be constant in time. Figure 39 compares the predicted average gas temperatures inside the tunnel. As expected, the gas temperature from the isothermal wall boundary condition with a maximum value of $644{ }^{\circ} \mathrm{R}$ is much smaller than that from the adiabatic boundary condition, which has a maximum value of $1,814^{\circ} \mathrm{R}$.

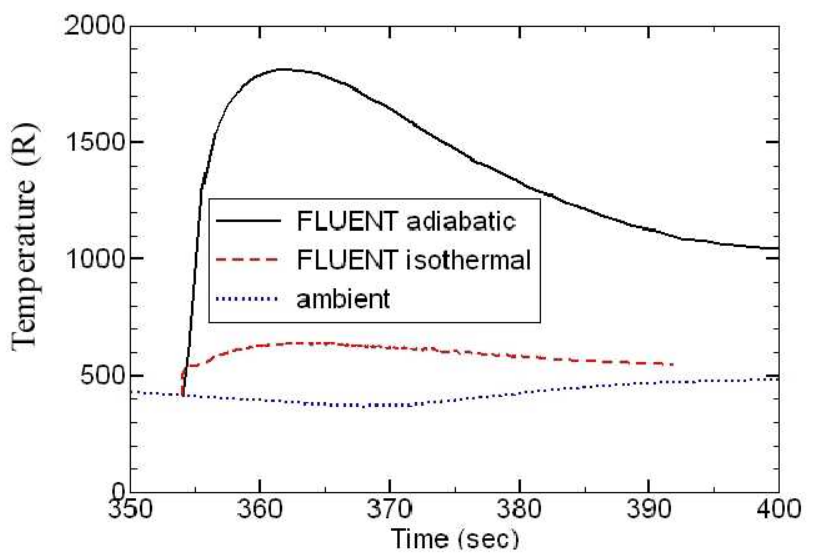

Figure 39. Predicted average temperature inside the tunnel.

Figure 40 shows the predicted gas temperature at the vent as well as the ambient static temperature, stagnation temperature, and recovery temperature. The recovery temperature is calculated from the ambient temperature using a recovery factor of 0.91 . The vent temperature for the adiabatic boundary condition is very close to the stagnation temperature after $360 \mathrm{sec}$ whereas that from the isothermal boundary condition is much smaller. The maximum temperature at the vent using the isothermal wall boundary condition is about $1,000{ }^{\circ} \mathrm{R}$, which is much smaller than the value of $2,350{ }^{\circ} \mathrm{R}$ using the adiabatic boundary condition. Note that the CFD simulations start at $354 \mathrm{sec}$ and the gas temperature at that time is assumed to be the same as the ambient static temperature. Otherwise, the gas temperature at $354 \mathrm{sec}$ should be much higher.

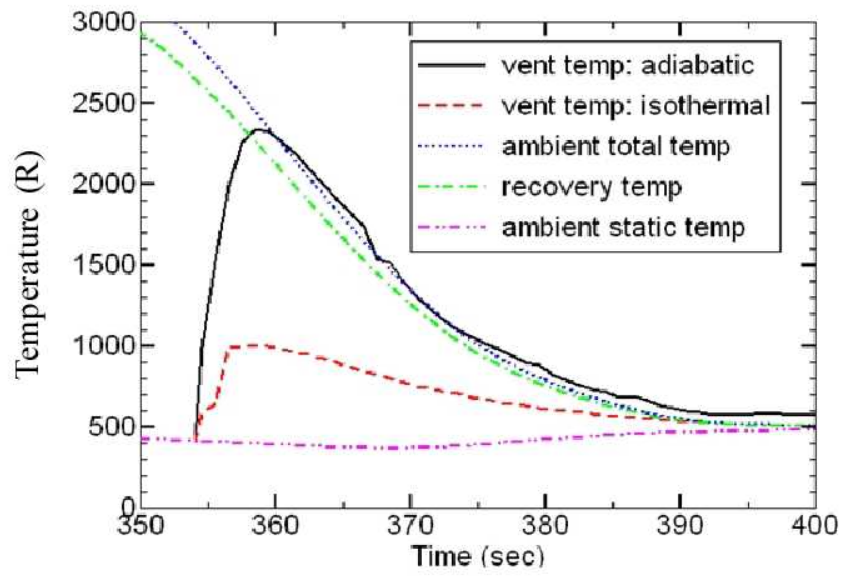

Figure 40. Predicted gas temperature at the vent and ambient temperature.

\section{Comparison Between 0D Lumped Parameter and 3D CFD Results}

As discussed above, the 0D lumped parameter analyses shown in Section II used all 1,000 trajectories whereas the 3D transient CFD analyses in Section III only used the mean trajectory to reduce the run time. In order to compare results from CFD with those from CHCHVENT, CHCHVENT is run again using the mean trajectory and adiabatic thermal boundary conditions. That is, the only difference between the burst and crush compartment pressures is due to the discharge coefficients applied. Results from CHCHVENT and CFD are compared in this section. 


\section{A. Comparison of Ascent Venting Results}

Figure 41 and Figure 42 show the comparison of pressure and temperature from CFD with those from CHCHVENT during ascent. In general, the average pressure and temperature from CFD are between the burst and crush results from CHCHVENT. CFD results agree better with the burst case than the crush case indicating that the crossflow dependent discharge coefficients are more applicable than the pressure dependent discharge coefficients.
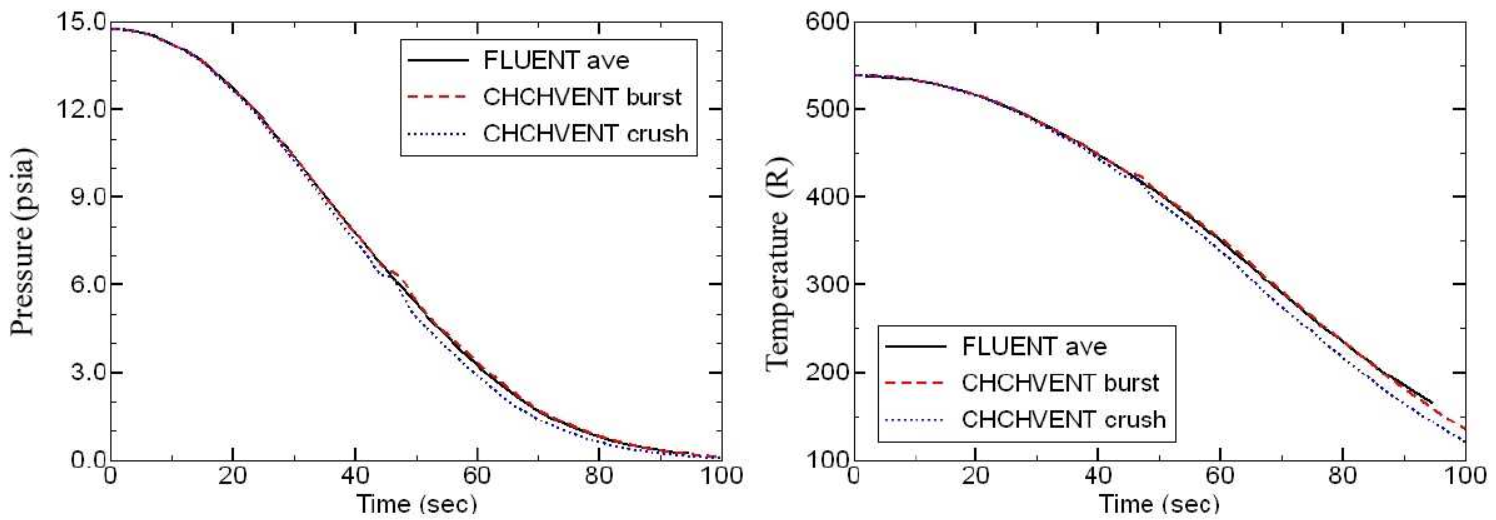

Figure 41. Comparison of pressure and temperature from CFD with those from CHCHVENT.

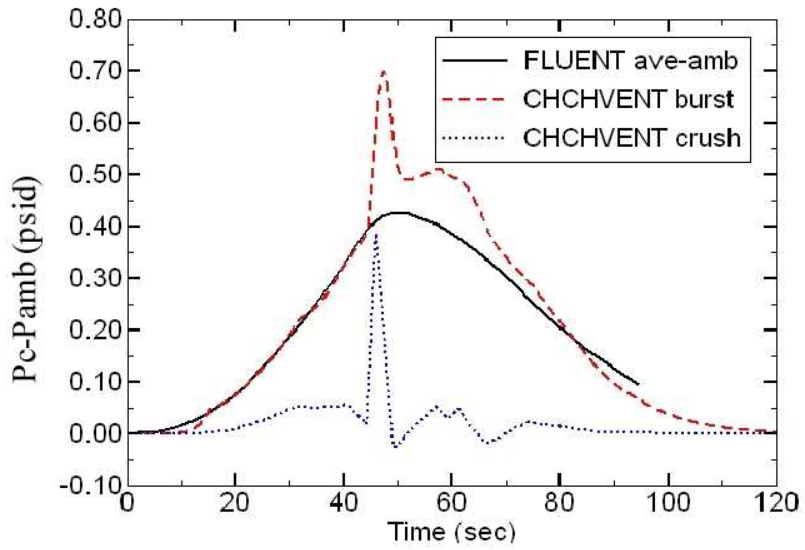

Figure 42. Comparison of pressure relative to freestream from CFD with that from CHCHVENT.

\section{B. Comparison of Reentry Venting Results}

Figure 43 shows the comparison of pressure and temperature from CFD with those from CHCHVENT during reentry. Similar to ascent, the average pressure from CFD is between the burst and crush pressures from CHCHVENT. The predicted average temperature from FLUENT is between those for the burst and crush cases from CHCHVENT after $370 \mathrm{sec}$ and larger at early times.
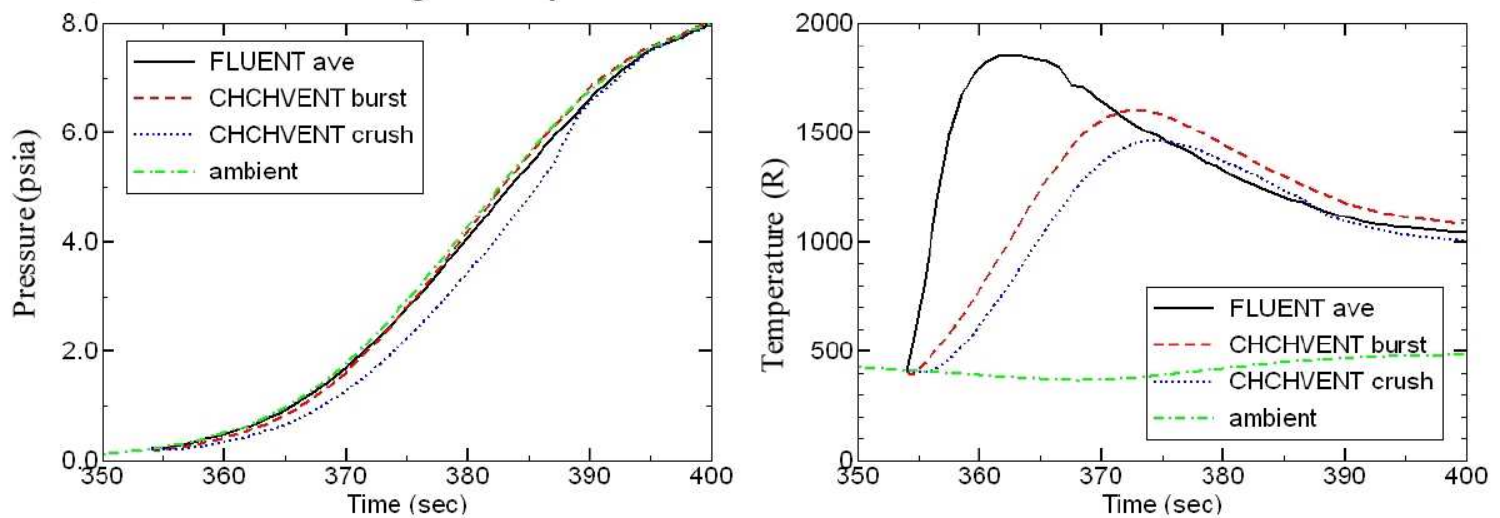

Figure 43. Predicted pressures and temperatures from FLUENT and CHCHVENT during reentry. 


\section{Conclusions}

Compartment venting analyses have been performed for the Ares I first stage systems tunnel using both the 0D lumped parameter method and the 3D transient CFD approach. The lumped parameter method assumes the gas pressure and temperature inside the systems tunnel are spatially uniform, which are questionable since the tunnel is about 1,700 inches long and 4 inches wide. Therefore, 3D transient CFD simulations using the commercial CFD code FLUENT were performed in order to examine the gas pressure and temperature variations inside the tunnel.

The lumped parameter method uses different modeling assumptions to predict the worst case skin differential pressures, which are defined as the difference between pressure inside the compartment and the local external pressure at the wall. For the burst case corresponding to maximum skin differential pressure, venting models were created to maximize the internal compartment pressures during flight. Minimum external wall pressures were coupled to these models to provide maximum burst loads. For the crush case corresponding to minimum skin differential pressure, models providing minimum internal compartment pressures were coupled with maximum external wall pressures. It was found that the 95 percentile skin differential pressure during ascent is 1.04 psid for the burst case and -2.70 psid for the crush case. During reentry, this differential pressure is 9.37 and -14.29 psid for the burst and crush cases, respectively. The maximum pressure difference between compartment and ambient is about 0.75 psid during ascent and 6.5 psid during reentry.

Results from CFD simulations indicate that the uniform pressure and temperature assumptions inside the systems tunnel are valid during ascent. Specifically, the difference between maximum and minimum pressures inside the tunnel is smaller than $0.01 \mathrm{psid}$, which occurs near the vent location. Away from the vent, this difference is only about 0.0002 psid. The difference between maximum and minimum temperatures is less than $0.3{ }^{\circ} \mathrm{R}$. During reentry, the uniform pressure assumption is also reasonable although the difference between maximum and minimum pressures is larger than that during ascent. In particular, this pressure difference is less than 0.67 psid near the vent and 0.0013 psid away from the vent. In contrast to the pressure; however, the difference between maximum and minimum temperature is as much as $3,150{ }^{\circ} \mathrm{R}$, indicating that the uniform temperature assumption is not valid during reentry.

Velocity vectors and streamlines from CFD simulations suggest that, during reentry, there is only a very small amount of gas flowing from ambient to the vent. The gas flow is from the systems tunnel to one side of the vent and from the other side of the vent to the tunnel. This flow pattern is due to the small difference between pressures inside and outside the tunnel and is consistent with the small discharge coefficient measured in the wind tunnel testing. It was found that there is much more gas flowing from the ambient to the vent for higher pressure rising rate cases where the difference between ambient pressure and tunnel pressure is larger. CFD results for pitch angles of 45 and 90 degrees show that the flow is from ambient to the tunnel through the vent and there is no flow from the tunnel to the vent. During ascent, the gas flow is from the tunnel to the ambient through the vent and there is no flow from the vent to the tunnel.

The predicted temperature inside the systems tunnel is very high because the adiabatic wall boundary condition was applied and the heat transfer between gas and solid was neglected. The average gas temperature inside the tunnel is predicted to be smaller than $644^{\circ} \mathrm{R}$ using the isothermal wall boundary conditions whereas it is as high as $1,814{ }^{\circ} \mathrm{R}$ using the adiabatic wall boundary condition. The isothermal wall boundary condition also reduces the maximum gas temperature at the vent from about $2,350^{\circ} \mathrm{R}$ to $1,000{ }^{\circ} \mathrm{R}$.

Predicted pressure and temperature inside the systems tunnel from CFD are compared with those from the lumped parameter method using the NASA code CHCHVENT. During ascent, the average pressure and temperature inside the tunnel from CFD are between the burst and crush results from CHCHVENT. CFD results agree better with the burst case than the crush case because the crossflow dependent discharge coefficients are more applicable than the pressure dependent discharge coefficients. During reentry, the average pressure from CFD is also between the burst and crush pressures from CHCHVENT while the predicted average temperature from CFD is between those for the burst and crush cases from CHCHVENT at late times and larger at early times. While the CHCHVENT runs take a few minutes, the $3 \mathrm{D}$ transient $\mathrm{CFD}$ simulations take a few months for each trajectory on a Linux cluster with AMD Opteron $2.6 \mathrm{GHz}$ processors. 


\section{References}

1. Wang, Q. and Arner, S., “Ares I First Stage Venting Environment Data Book," ATK report TR022310, March 2008.

2. Heman, J., Stevens, L.M., and Wang, Q., "Ares I-X First Stage Thermal and Venting Design Data Book," ATK report TR018919 Rev. B, March 2009.

3. Downs, W.J., "Preliminary Ares I Ascent Venting Environments Data Book," CLV-DE-20300, NASA MSFC, August 31, 2007.

4. Systems Analysis and Integration Laboratory, "Solid Rocket Booster Venting Analysis," SE-019-142-2H, Revision A, NASA MSFC, July 1981.

5. Fay, John F., "Program CHCHVENT Version 5 User's Manual and Software Description," MSFC Report 631-001-93-007, November 1993.

6. Erickson, Gary E., "0.01-Scale CLV DAC-1 Pressure Model Testing at Supersonic Speeds in the NASA Langley Research Center unitary plan wind tunnel," NASA Langley Research Center, April 2007 


\section{Compartment Venting Analyses of the Ares I First Stage Systems Tunnel}

Qunzhen Wang and Stephen Arner

ATK Space Systems, Brigham City, UT 84302

45th AIAA/ASME/SAE/ASEE Joint Propulsion Conference and Exhibit Colorado Convention Center, Denver, Colorado August 3, 2009

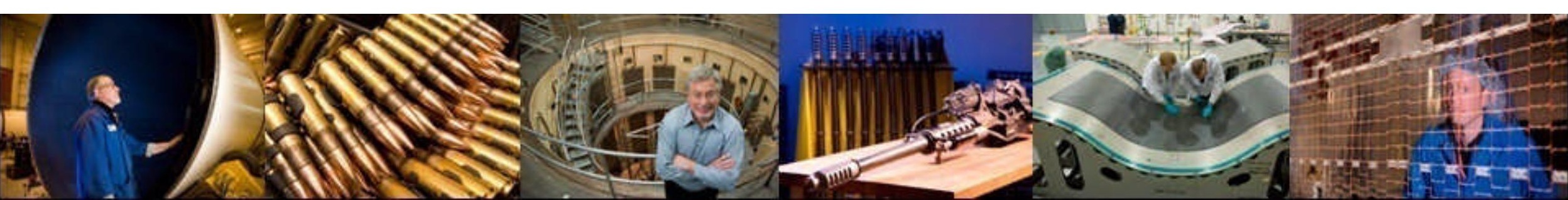




\section{Outline}

\section{Background}

Lumped parameter analyses

- Trajectories

- Pressure coefficients

- Discharge coefficients

- Ascent and reentry

3D transient CFD analyses

- Ascent

- Reentry

Comparison between lumped parameter and CFD results

Conclusions 


\section{Background: Compartment Venting Analyses}

Several compartments in the Ares I Crew Launch Vehicle contain trapped gases that will escape through vents and/or leak paths during flight

- During ascent, the freestream pressure decreases with time. The pressures inside the vented compartments also decrease, but lag the freestream pressure.

- Both the freestream pressure and pressure inside the compartments increase during reentry, but the internal pressure again lags the freestream pressure.

- Differential pressures across the vehicle walls are induced through all phases of flight.

Compartment venting analyses: predict skin differential pressures so the integrity of solid walls can be evaluated and properly designed

Lumped parameter method

- Assume gas pressure and temperature inside the compartment are spatially uniform

- Assume gas velocity inside the compartment is zero

- The gas flow is assumed to be one-dimensional, isentropic, and quasi-steady

- The gas is assumed to be inviscid and an empirical discharge coefficient is applied to account for viscous and other losses 


\section{Systems Tunnel Venting Analyses}

Ares I first stage systems tunnel is a vented compartment

- Systems tunnel is 1,700 in. long and 4 in. wide

- Are uniform pressure and temperature assumptions still valid inside such a long narrow tunnel?

Perform 3D transient CFD simulations to assess the uniform pressure and temperature assumptions

- Predict pressure and temperature at every point inside the systems tunnel

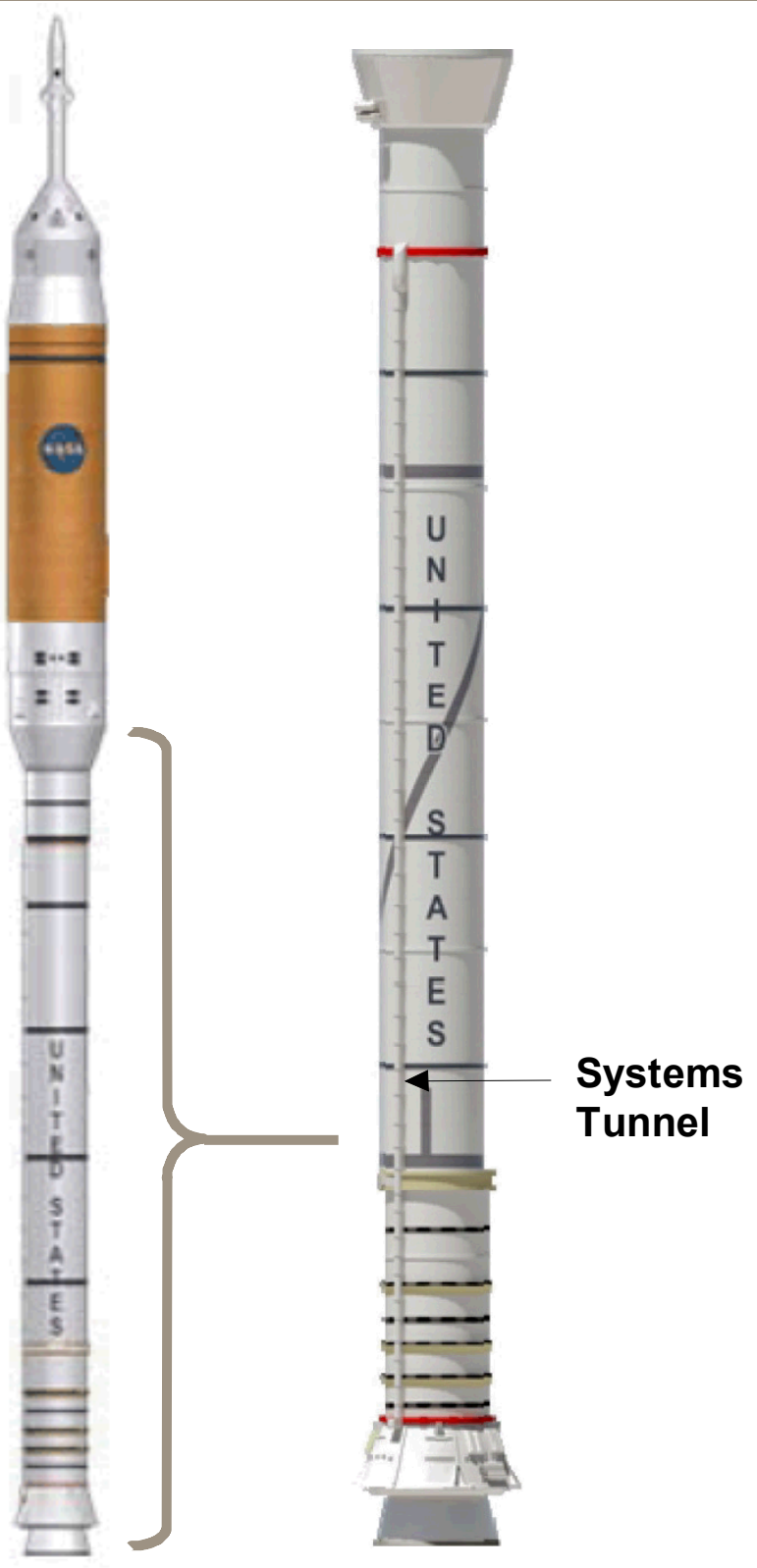




\section{Objectives}

Perform compartment venting analyses of the Ares I first stage systems tunnel using the lumped parameter method

- During ascent and reentry

Perform 3D transient CFD compartment venting analyses

- Assess the uniform pressure and temperature assumptions inside the systems tunnel

Compare results from CFD with those from the lumped parameter method 


\section{Lumped Parameter Method}

CHCHVENT: developed at NASA MSFC

Skin differential pressure: $d p_{\text {skin }}(t, x)=p_{C}(t)-C p\left(M_{\infty}, \theta, \varphi, x\right) \times q_{\infty}(t)-p_{\infty}(t)$

Run two cases for each trajectory

- Burst: maximum compartment pressure coupled with minimum external wall pressure

- Crush: minimum compartment pressure coupled with maximum external wall pressure

Discharge coefficients

- Pressure dependent

- Pressure and crossflow dependent

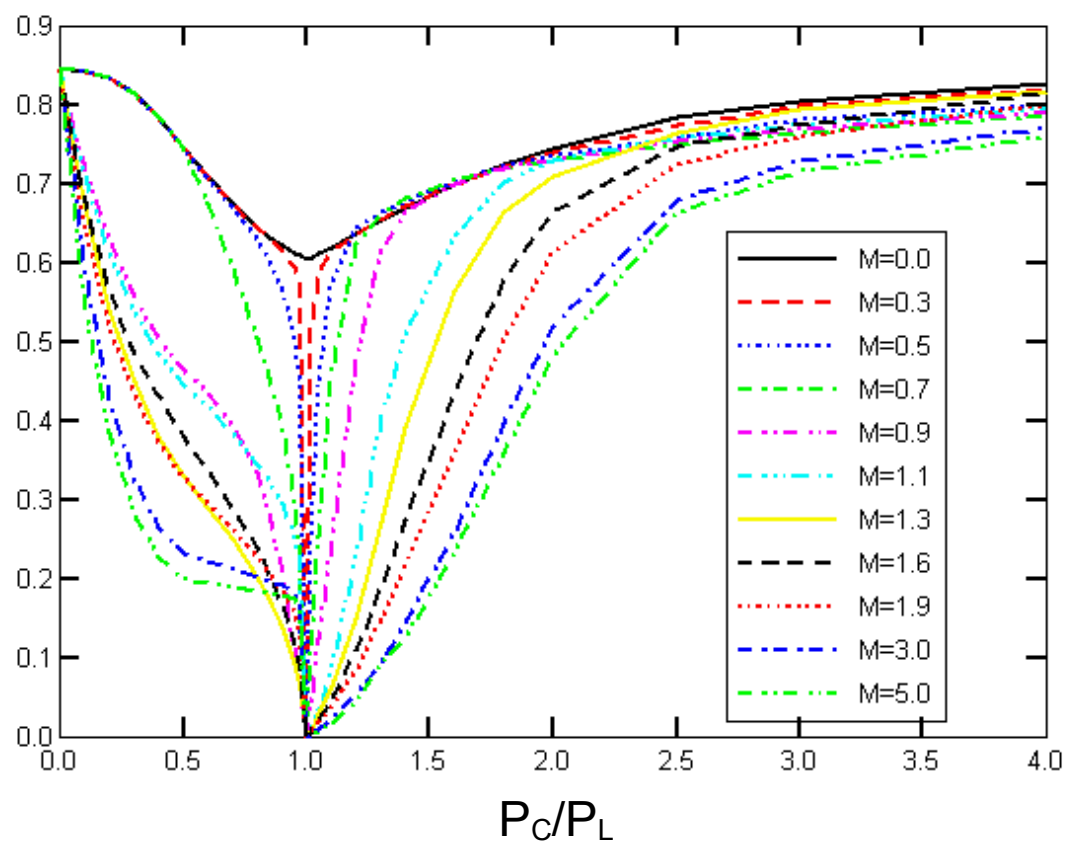




\section{Input Parameters}

Total compartment volume is $19 \mathrm{ft}^{3}$

A total of eight venting holes

- Each set of four holes is backed by a rain shield

- One screen is placed behind two vent holes for a total of four screens

Modeled as one hole with a venting area of $4.82 \mathrm{in}^{2}$ due to the rain shield

Area/volume (AV) ratio $=0.254 \mathrm{in}^{2} / \mathrm{ft}^{3}$

1,000 trajectories used for both ascent and reentry

Pressure coefficients

- Ascent: wind tunnel tests

- Reentry: CFD simulations 


\section{Mean Trajectory}

\section{ATK}

A premier aerospace and defense company
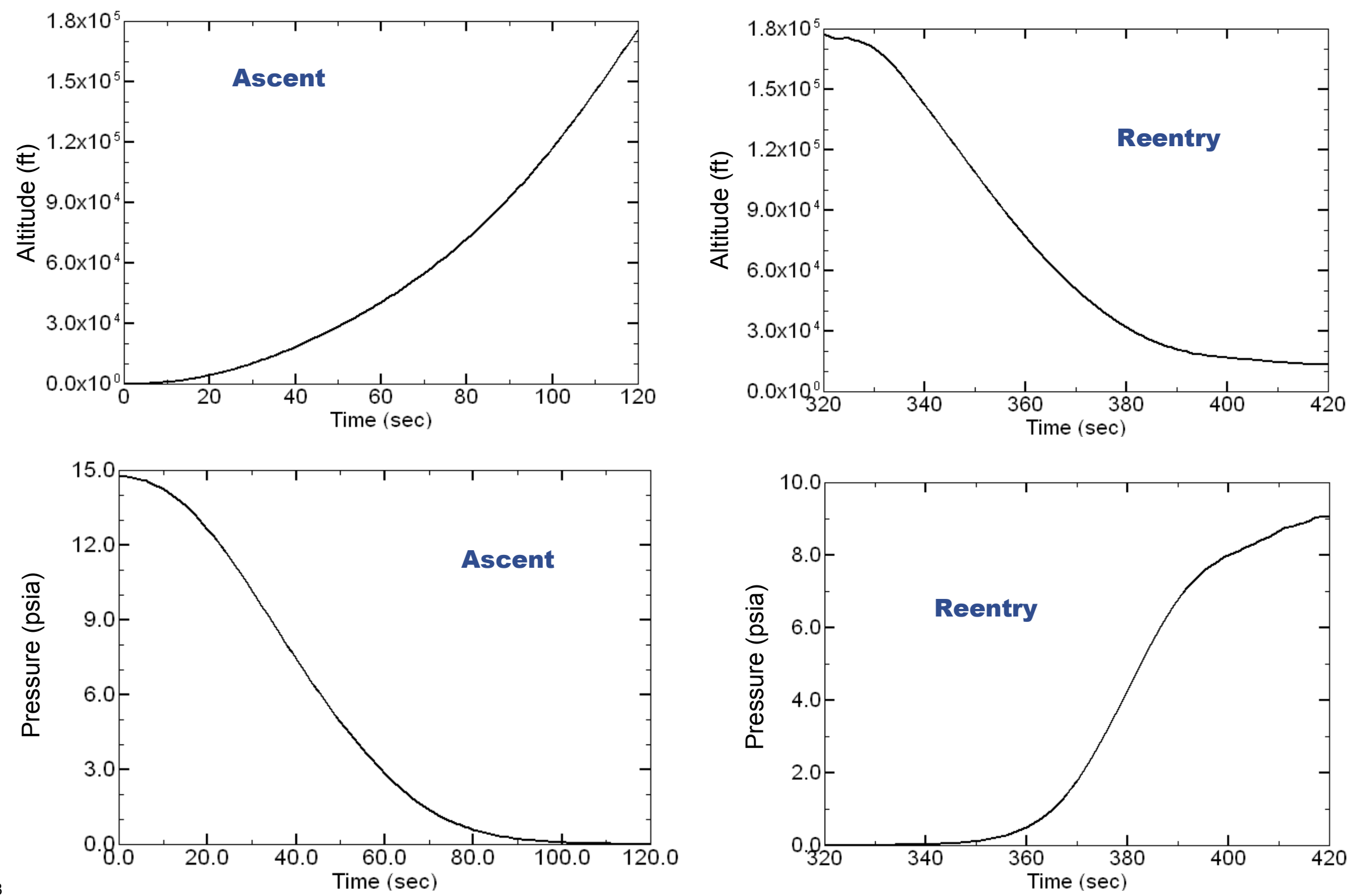


\section{Trajectories}

A premier aerospace and defense company
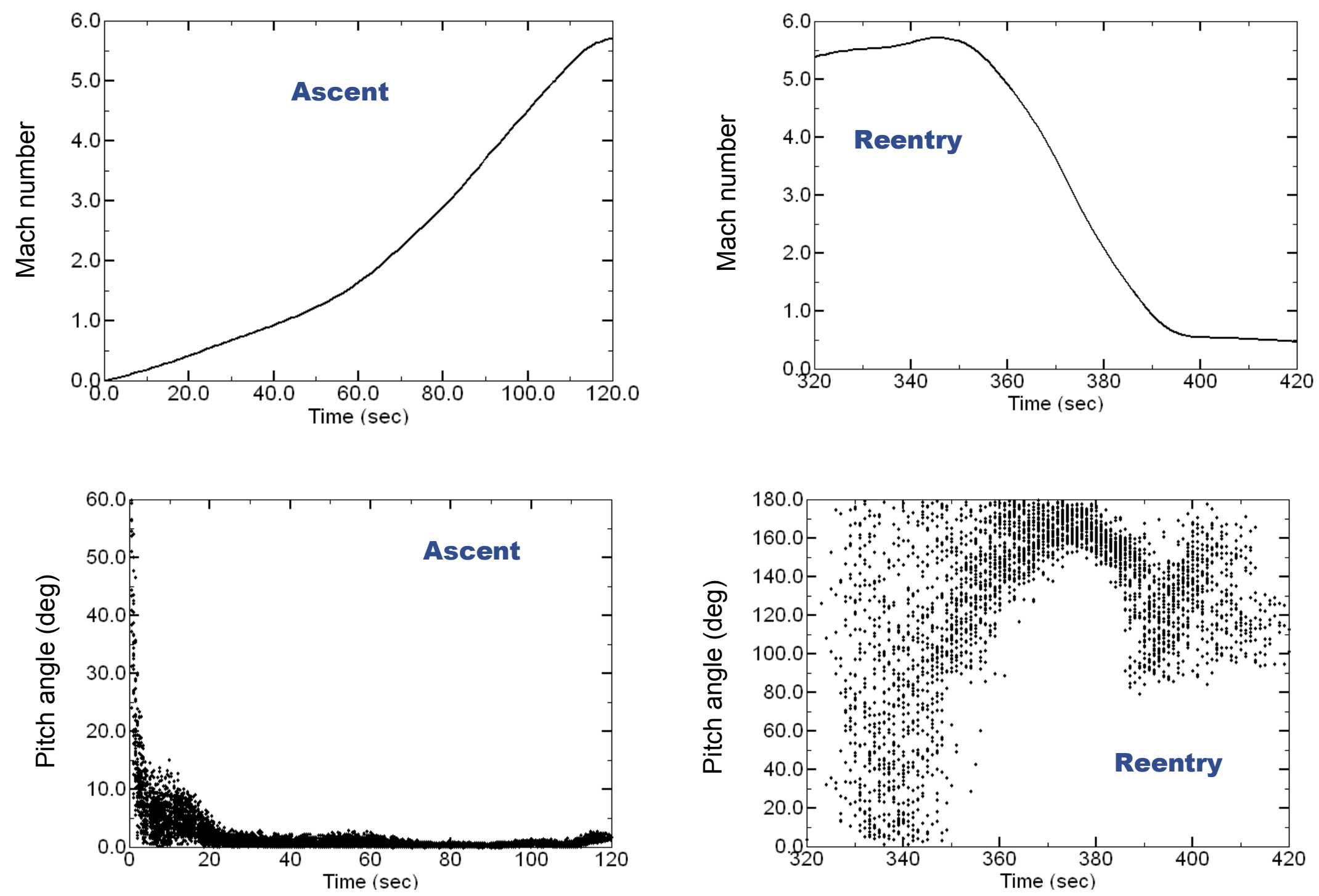


\section{Ascent Pressure from CHCHVENT}
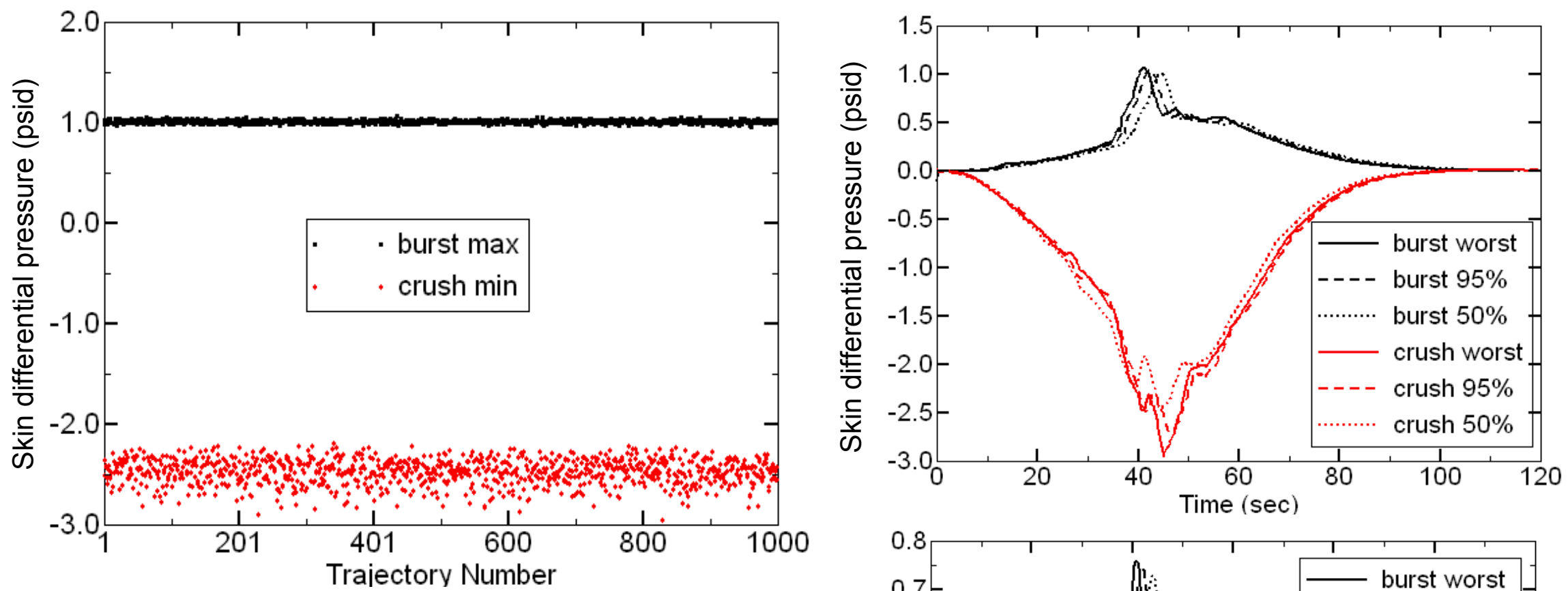

\begin{tabular}{|c|c|c|c|c|}
\hline \multirow{2}{*}{ worst } & \multicolumn{2}{|c|}{ burst } & \multicolumn{2}{c|}{ crush } \\
\cline { 2 - 5 } & $\mathrm{dp}($ psid) & traj No. & $\mathrm{dp}$ (psid) & traj No. \\
\hline 95th percentile & 1.06 & 436 & -2.95 & 828 \\
\hline 50th percentile & 1.04 & 412 & -2.70 & 218 \\
\hline
\end{tabular}

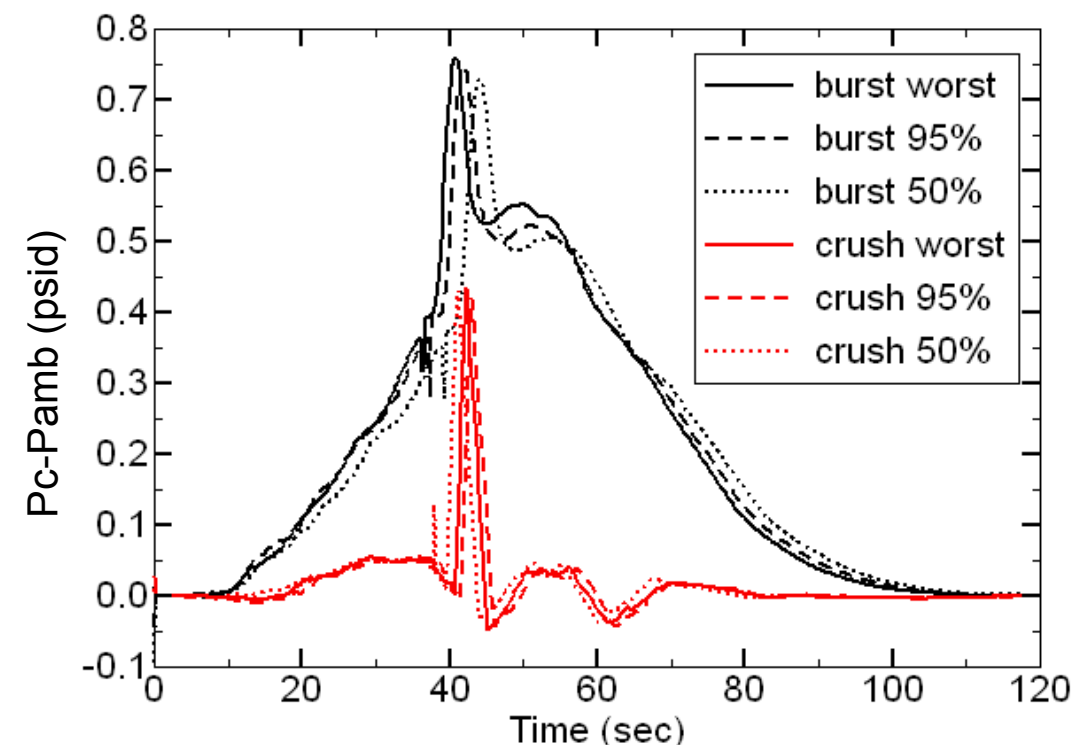




\section{Ascent Pressure: Compartment, Vent, and Ambient (ATK)}

A premier aerospace and defense company
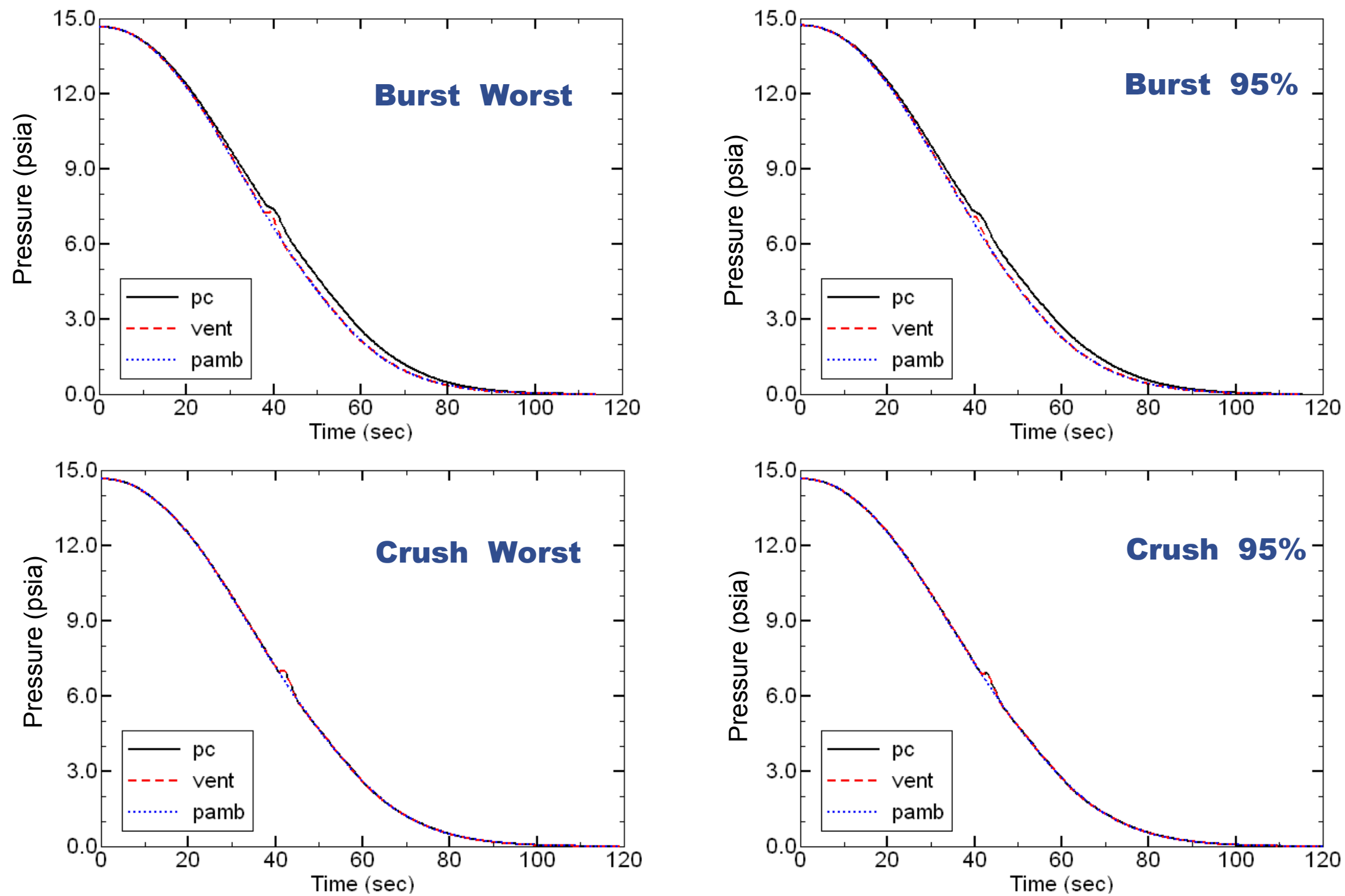


\section{Reentry Pressure from CHCHVENT}
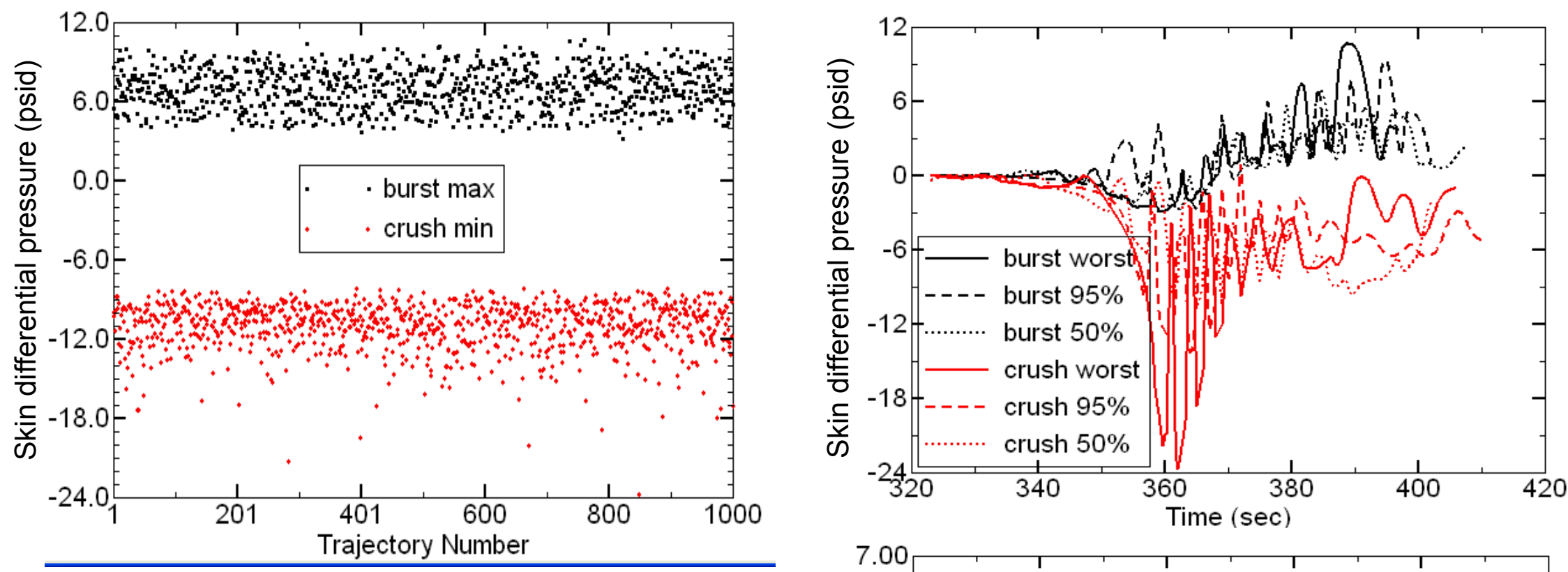

\begin{tabular}{|c|c|c|c|c|}
\hline \multirow{2}{*}{} & \multicolumn{2}{|c|}{ burst } & \multicolumn{2}{c|}{ crush } \\
\cline { 2 - 5 } & $\mathrm{dp}$ (psid) & traj No. & $\mathrm{dp}$ (psid) & traj No. \\
\hline worst & 10.68 & 760 & -23.72 & 849 \\
\hline 95th percentile & 9.37 & 417 & -14.29 & 514 \\
\hline 50th percentile & 6.93 & 691 & -10.66 & 308 \\
\hline
\end{tabular}

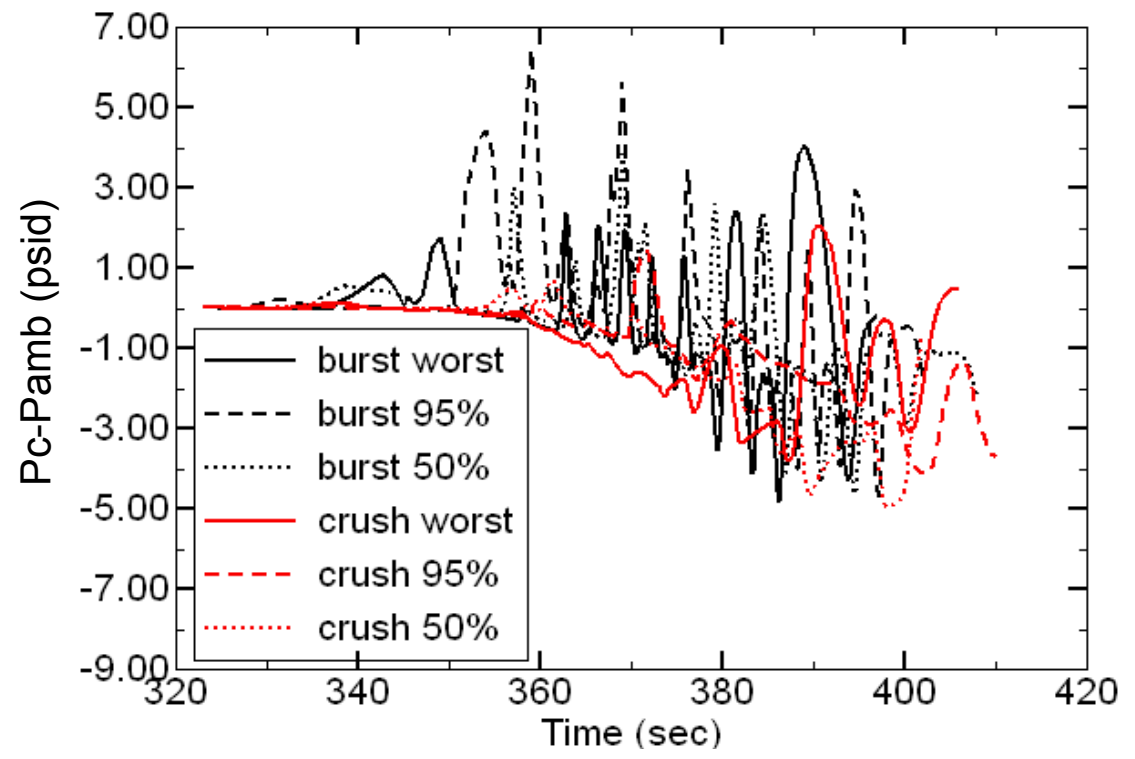




\section{Reentry Pressure: Compartment, Vent, and Ambient (ATK}

A premier aerospace and defense company
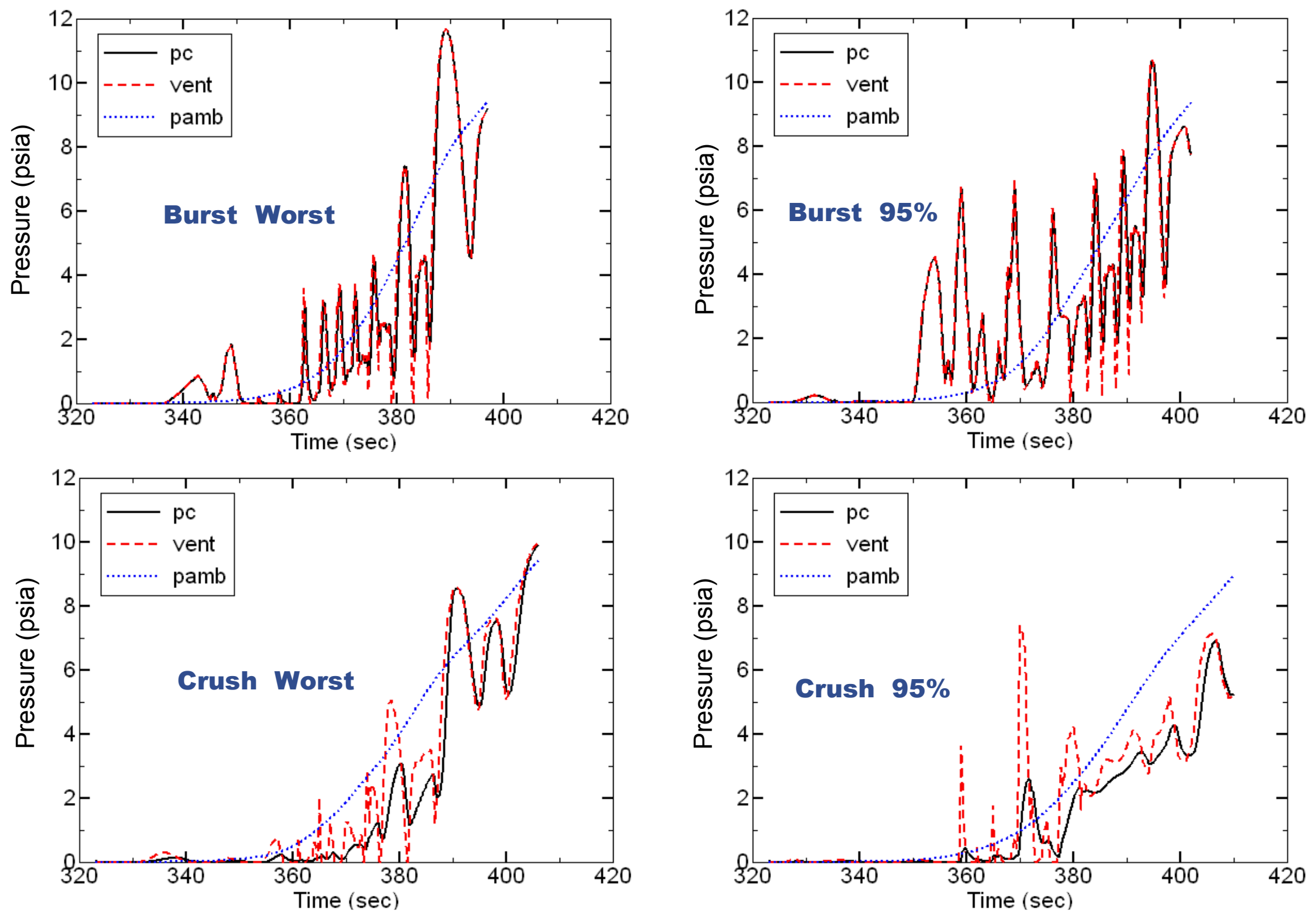


\section{CFD Simulation}

\section{Commercial CFD code FLUENT}

3D transient

Laminar flow: gas velocity inside the tunnel is very low

Assume all pitch angles are zero

Geometry is simplified

- There are eight venting holes, two rain shields, and four screens

- Modeled as one venting hole with an area equal to the minimum venting area due to the rain shield

- Rain shields and screens are not modeled

- Individual cables inside the tunnel are not modeled

- Assume the cross section geometry is the same at different axial locations

Only model half of the tunnel due to symmetry

About 1.32 million quadrilateral flow cells 
CFD Model

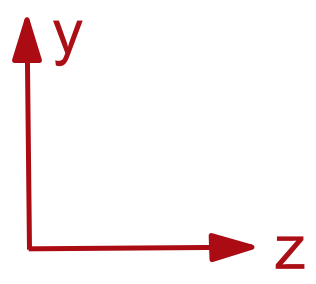

Symmetric plane

$\downarrow$

Flight Cable

Preliminary Layout: Aft Segment

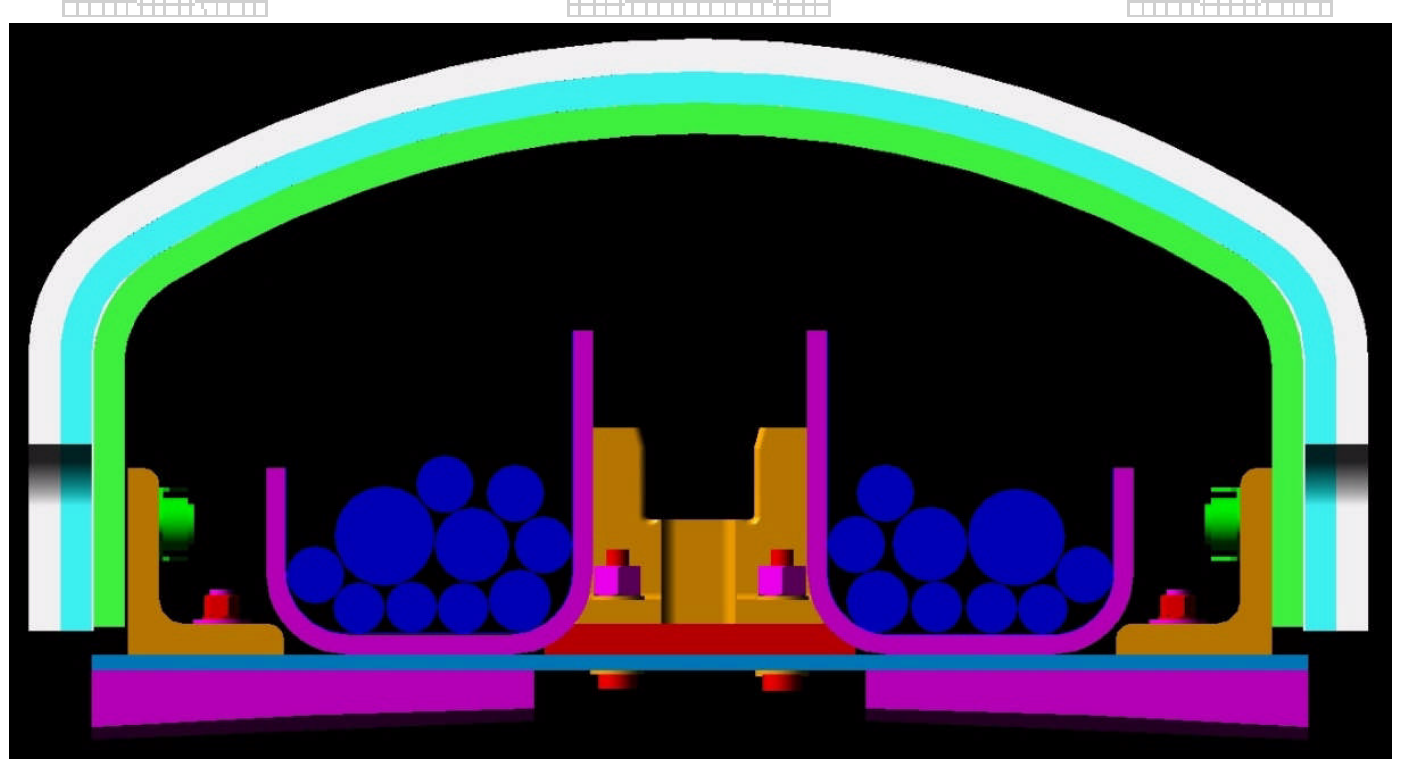




\section{Geometry}

\section{ATK}

A premier aerospace and defense company

\section{Vent Size}

2.41 in. $\times 1$ in.

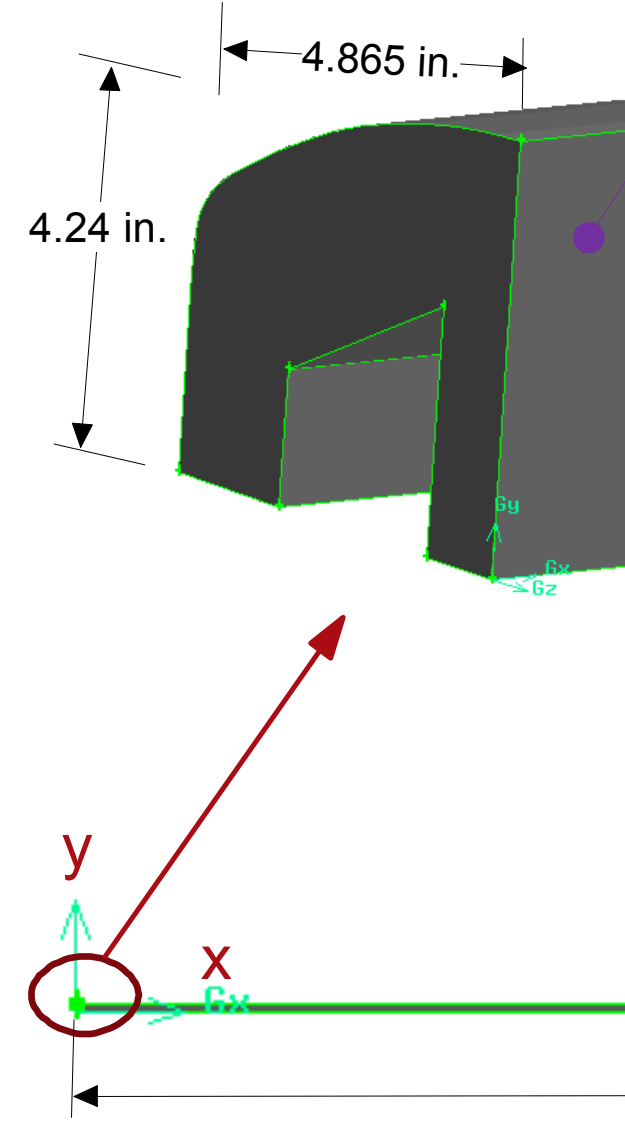

Symmetric Plane

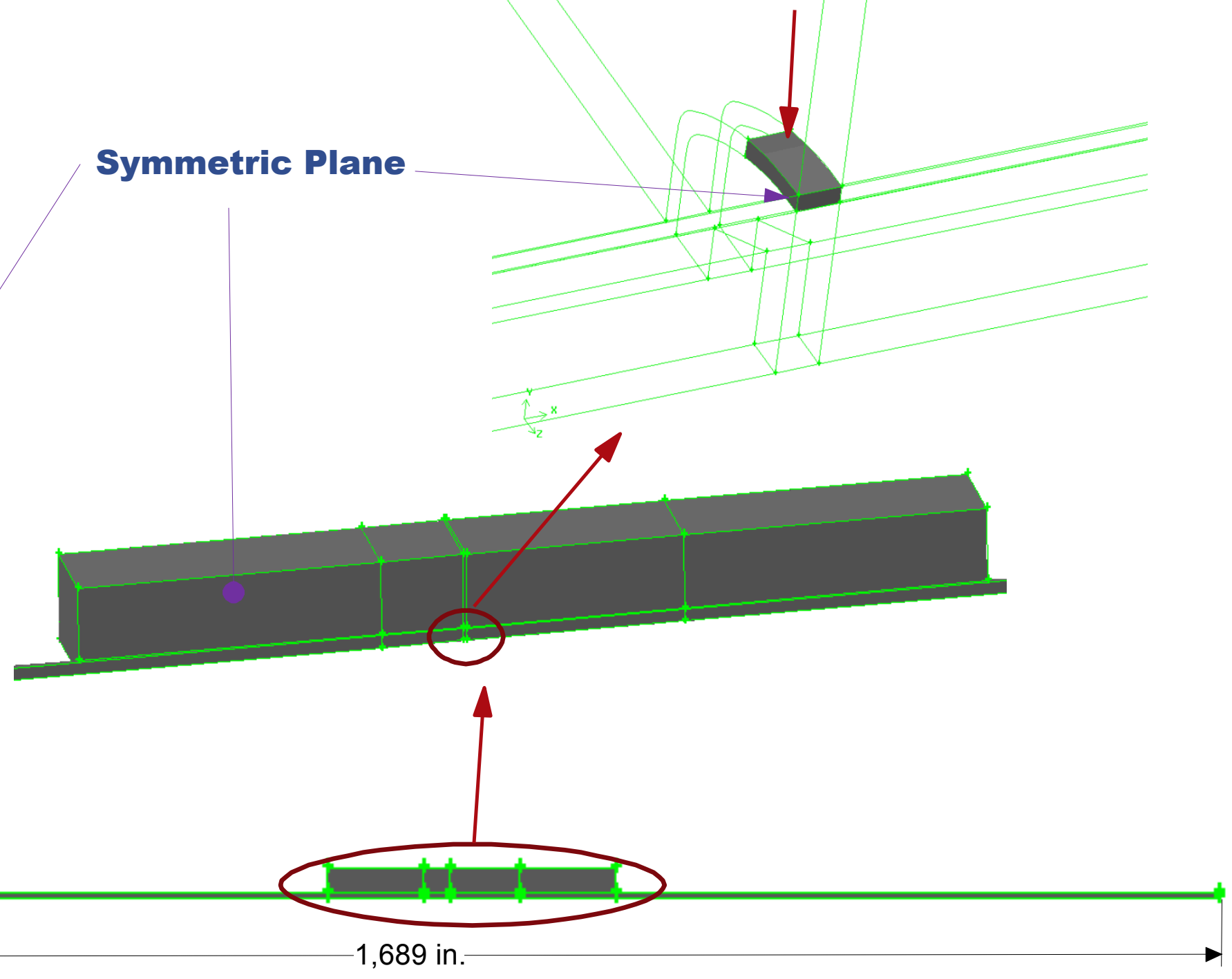




\section{Geometry and Grid}

Computational domain includes

- Full length of the tunnel

- Vent

- External area near the vent

\section{x-y Symmetric Plane}

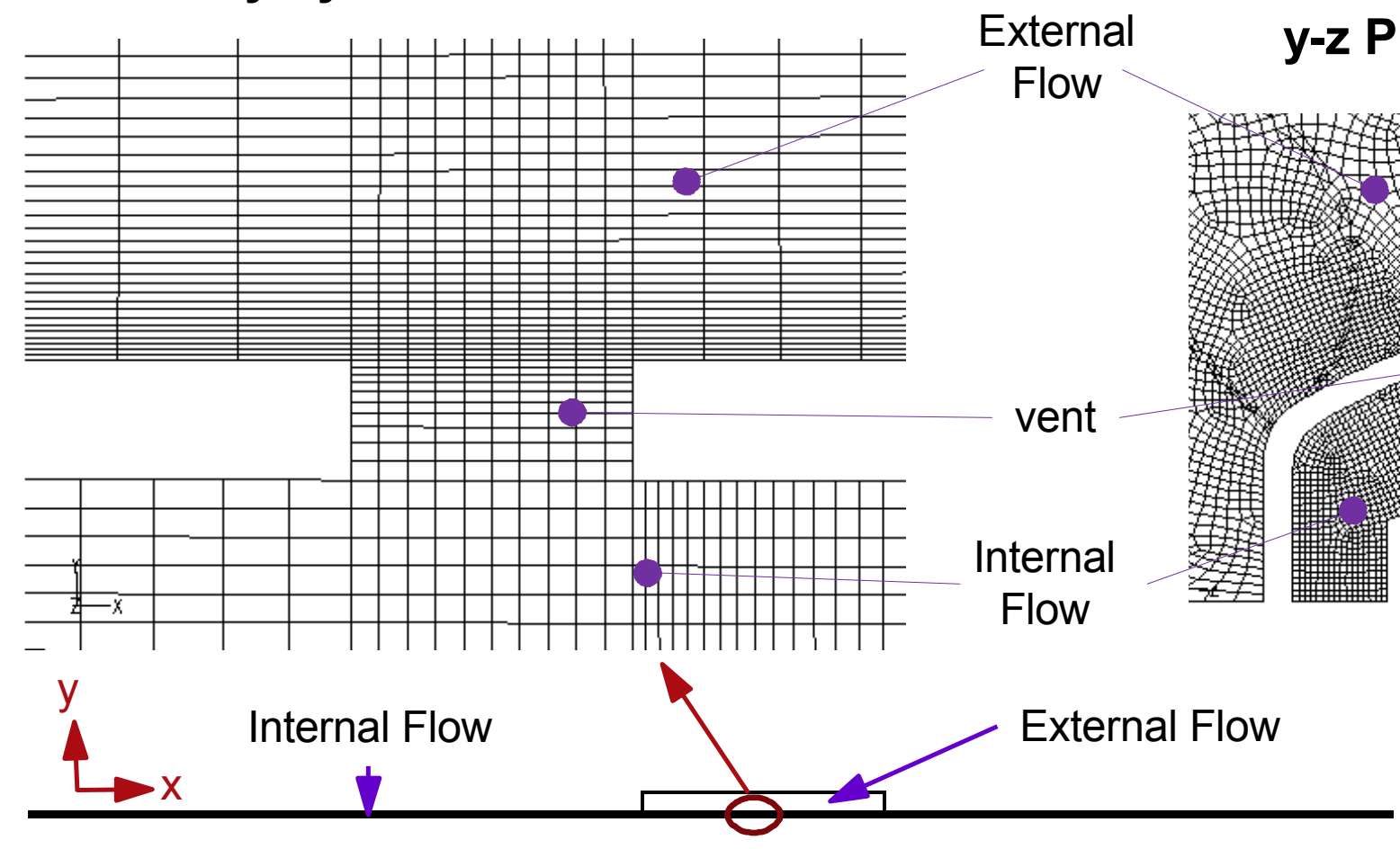

y-z Plane at Vent Center

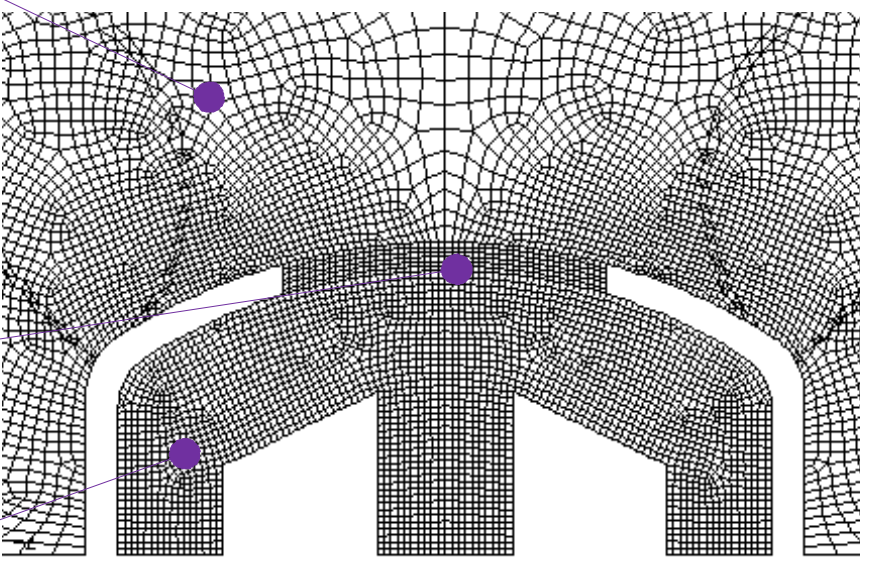




\section{Ascent: Pressure and Temperature Inside Tunnel (ATK}

A premier aerospace and defense company
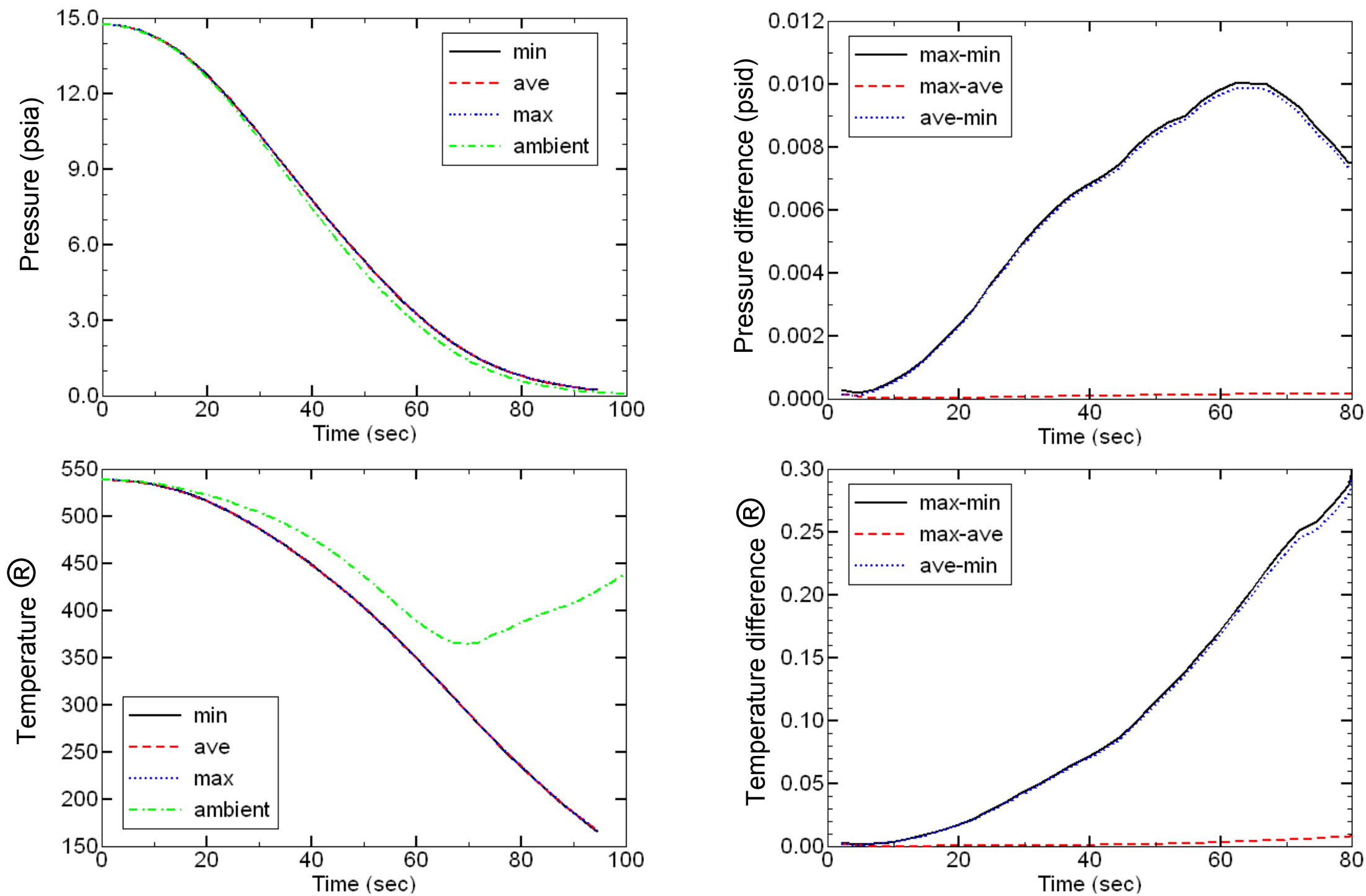


\section{Ascent: Pressure at All Cells Inside the Tunnel}

Uniform pressure and temperature assumptions inside the systems tunnel are valid during ascent

- The difference between maximum and minimum pressures is less than 0.01 psid

- Away from the vent, the difference is less than 0.0002 psid

- The difference between maximum and minimum temperatures is less than $0.7^{\circ} \mathrm{R}$
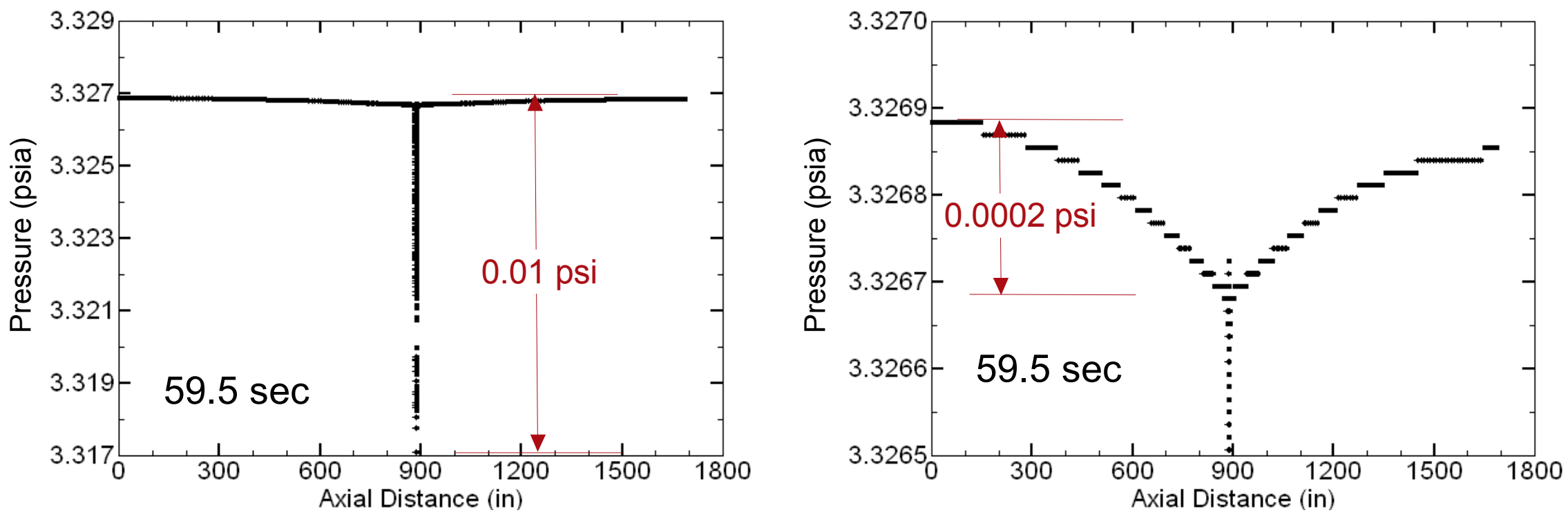


\section{Ascent: Pressure at the Symmetric Plane}

A premier aerospace and defense company

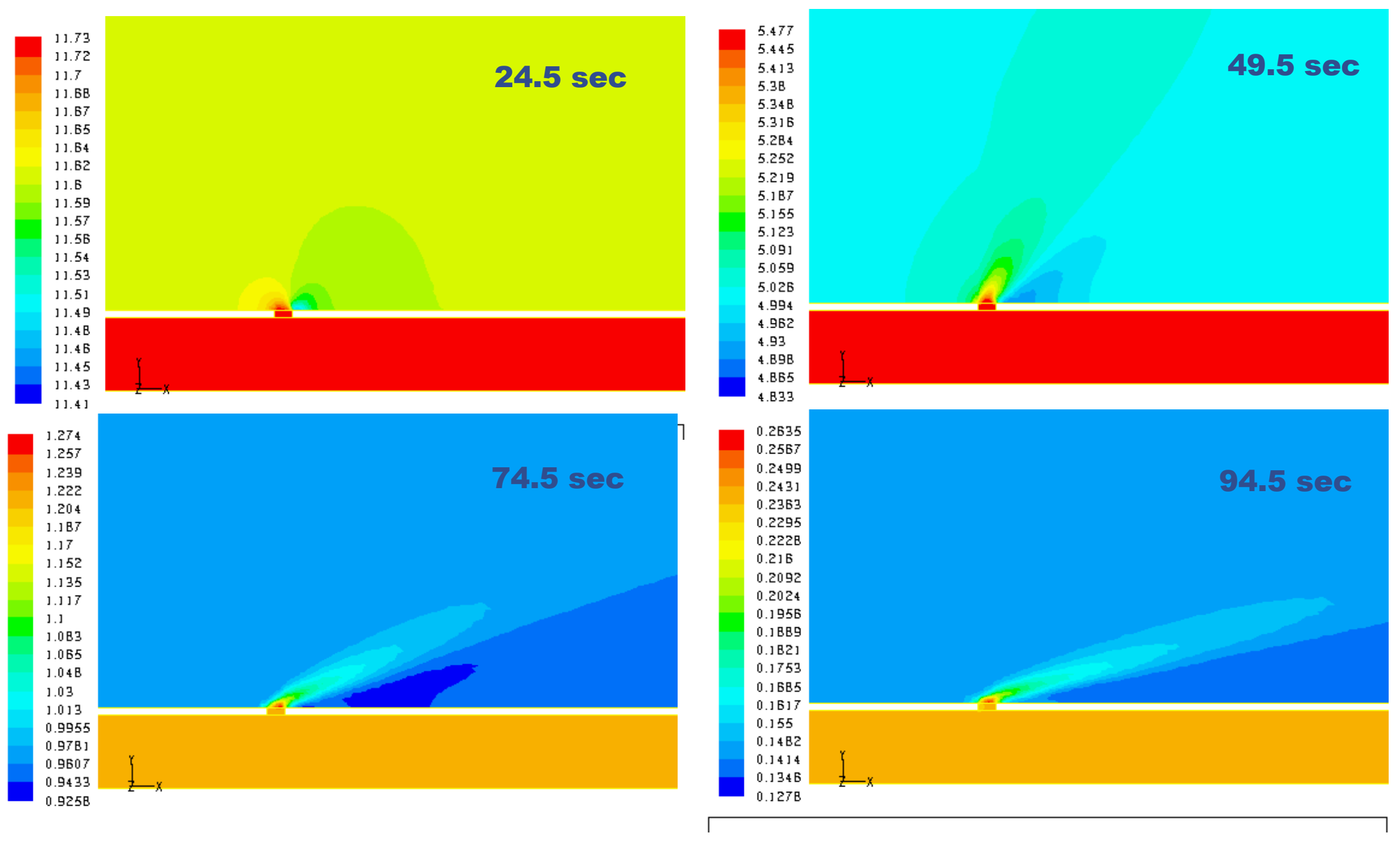




\section{Ascent: Pressure at the Cross Section y-z Plane ATK}

A premier aerospace and defense company
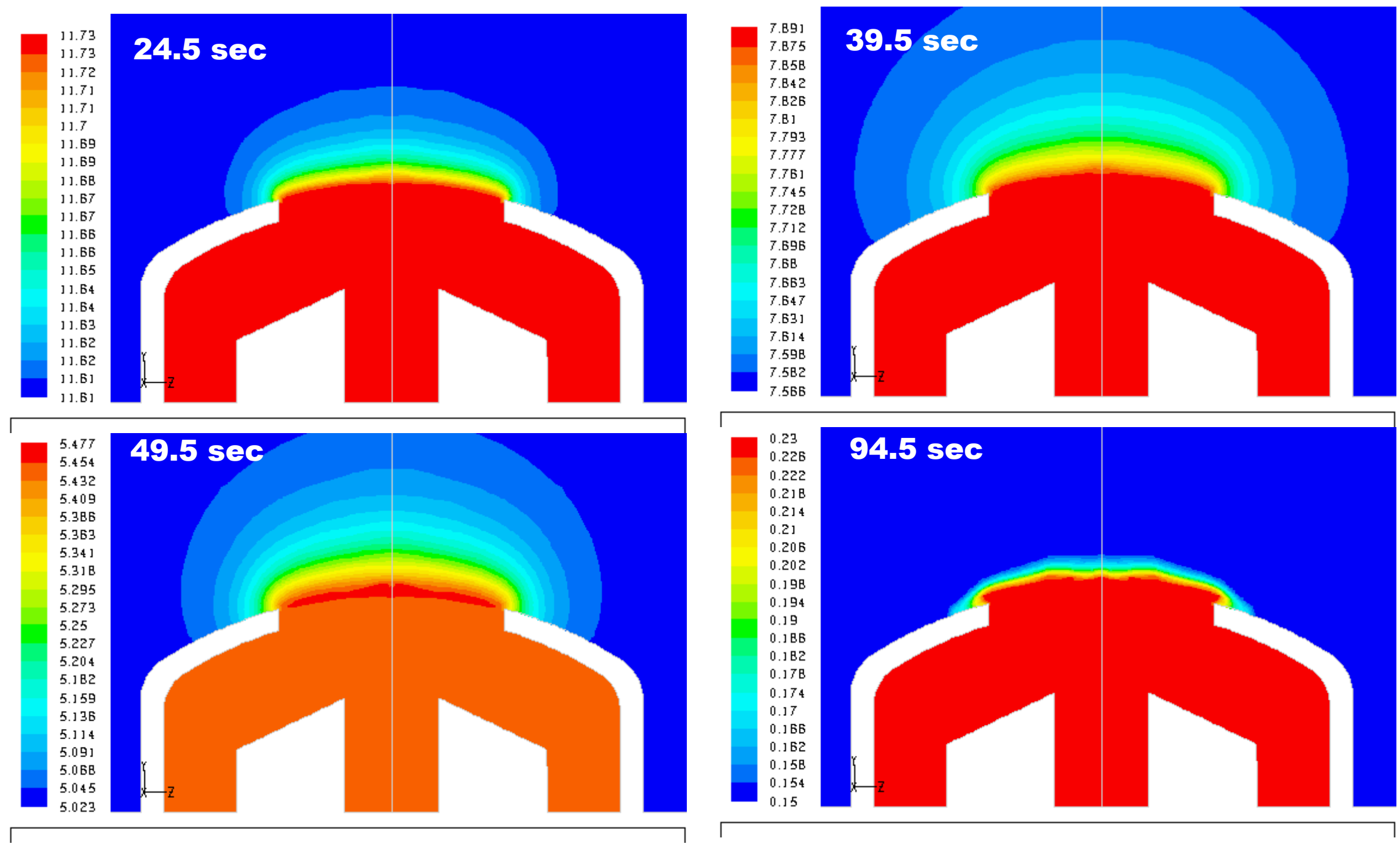


\section{Ascent: Velocity Vector and Streamlines: $x-y$ Plane (ATK}

A premier aerospace and defense company
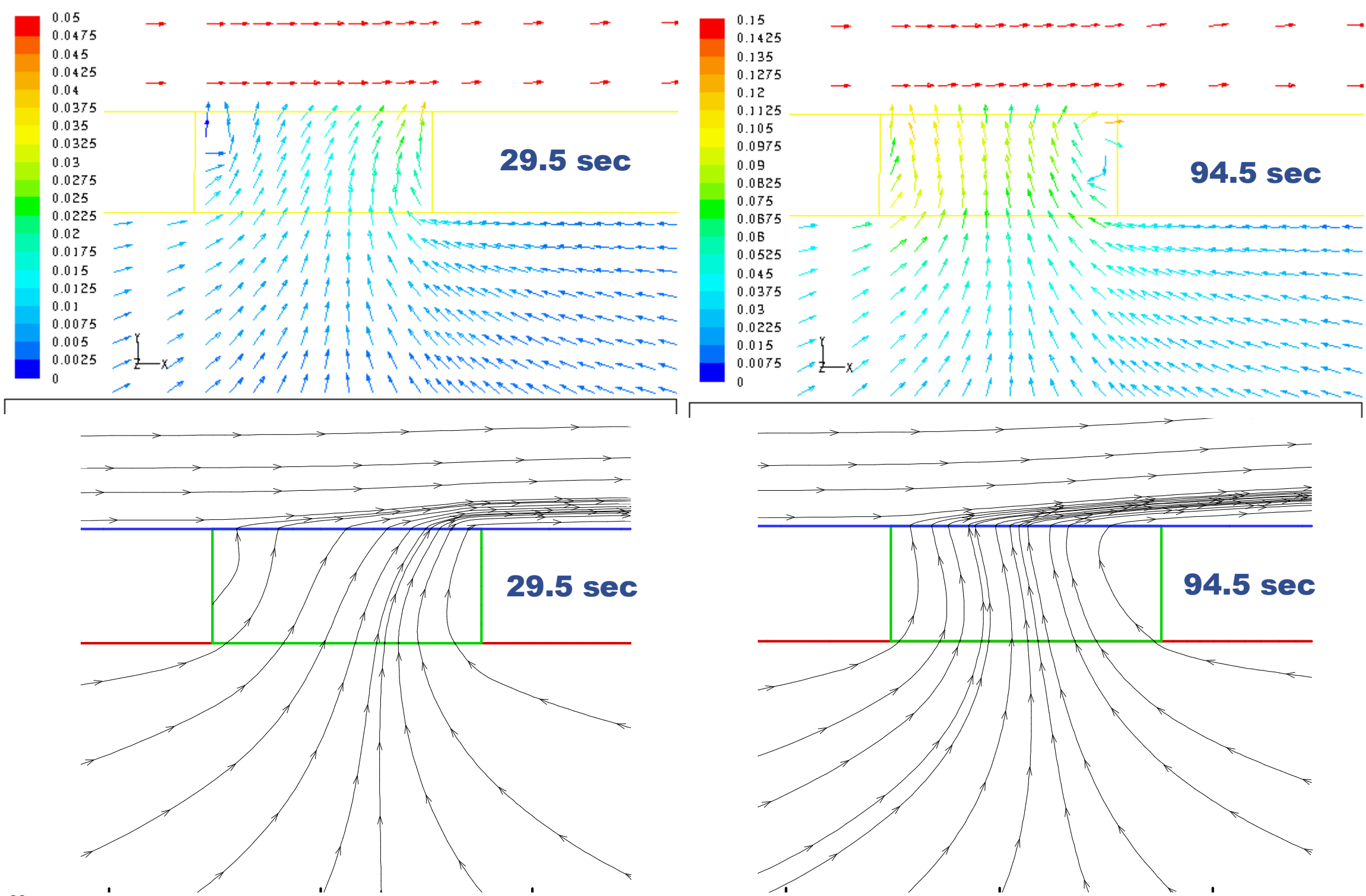


\section{Reentry: Pressure and Temperature Inside Tunnel}

A premier aerospace and defense company
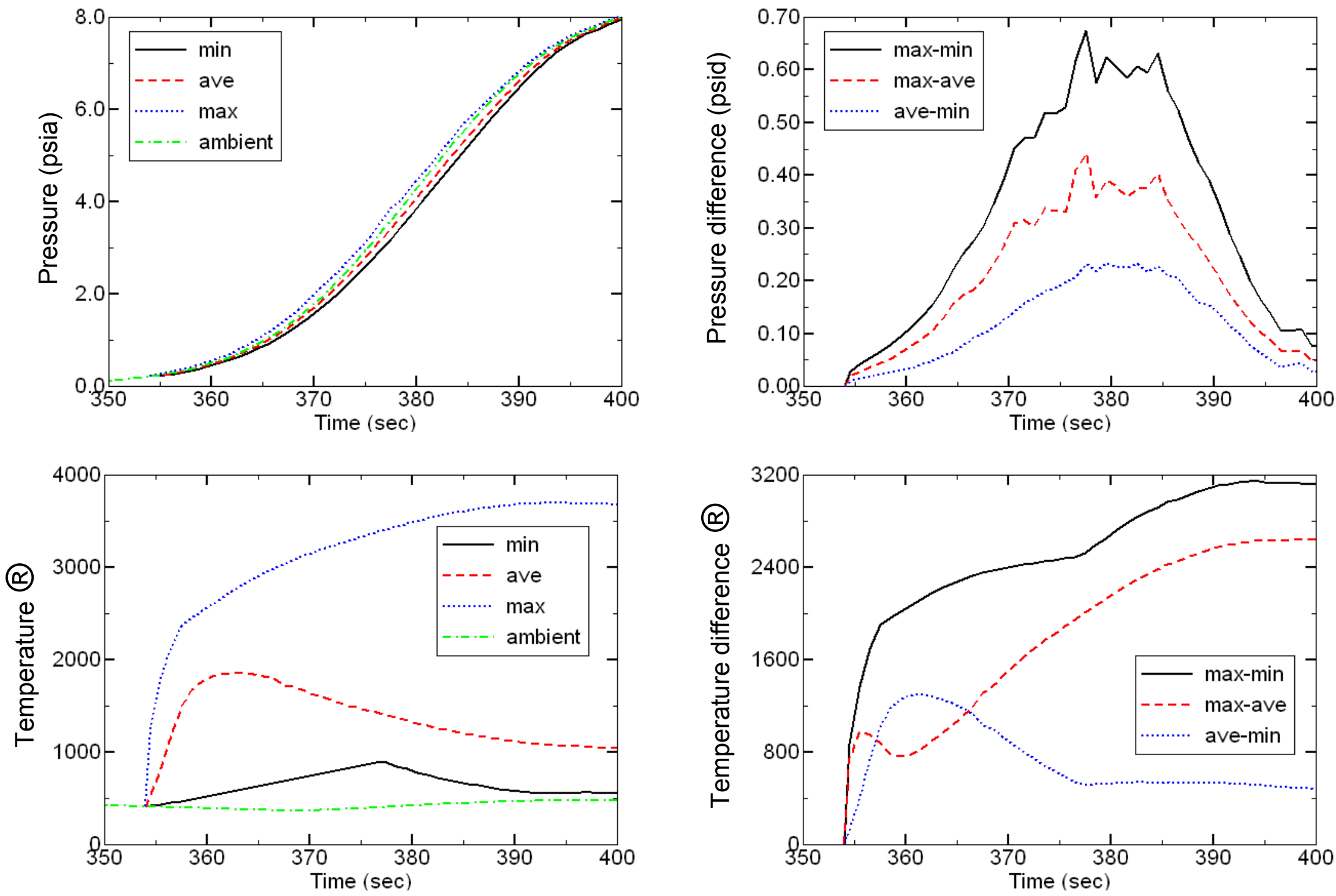


\section{Reentry: Pressure at All Cells at $\mathbf{3 7 6 . 5} \mathrm{sec}$}

Uniform pressure assumption inside the tunnel is reasonable during reentry

- The difference between maximum and minimum pressures is less than 0.62 psid

- Away from the vent, the difference is less than 0.0013 psid

Uniform temperature assumption inside the tunnel is not valid during reentry

- The difference between maximum and minimum temperatures is as much as $3,150{ }^{\circ} \mathrm{R}$
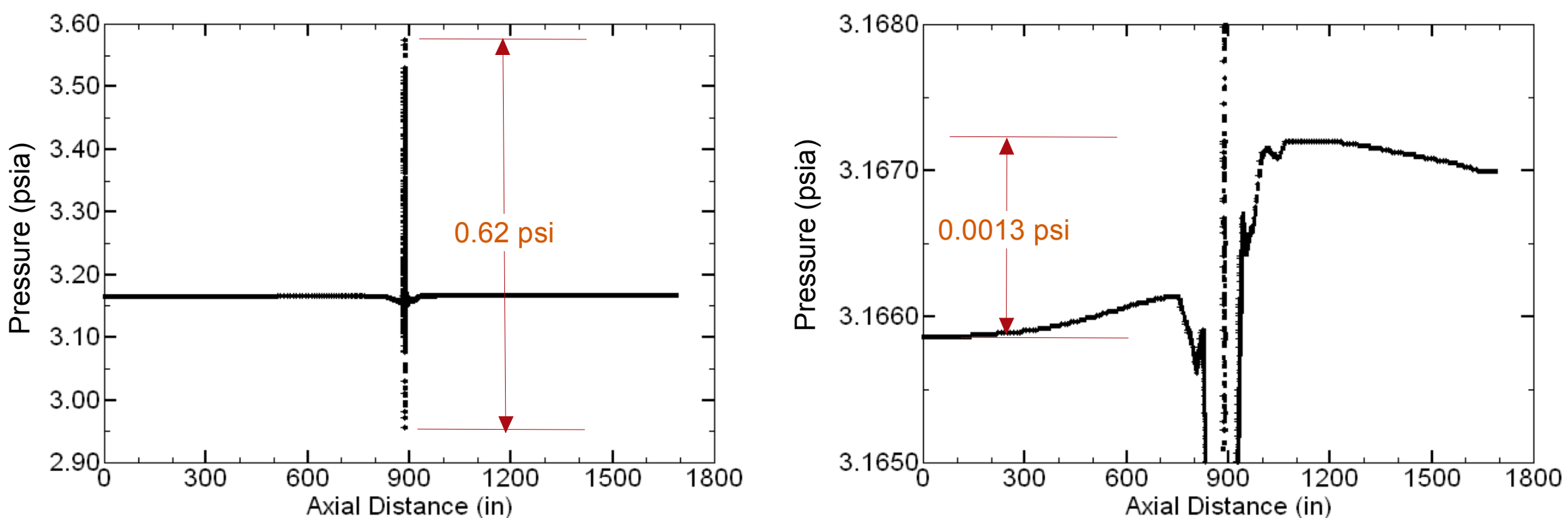


\section{Reentry: Velocity Vector and Streamlines}

A premier aerospace and defense company
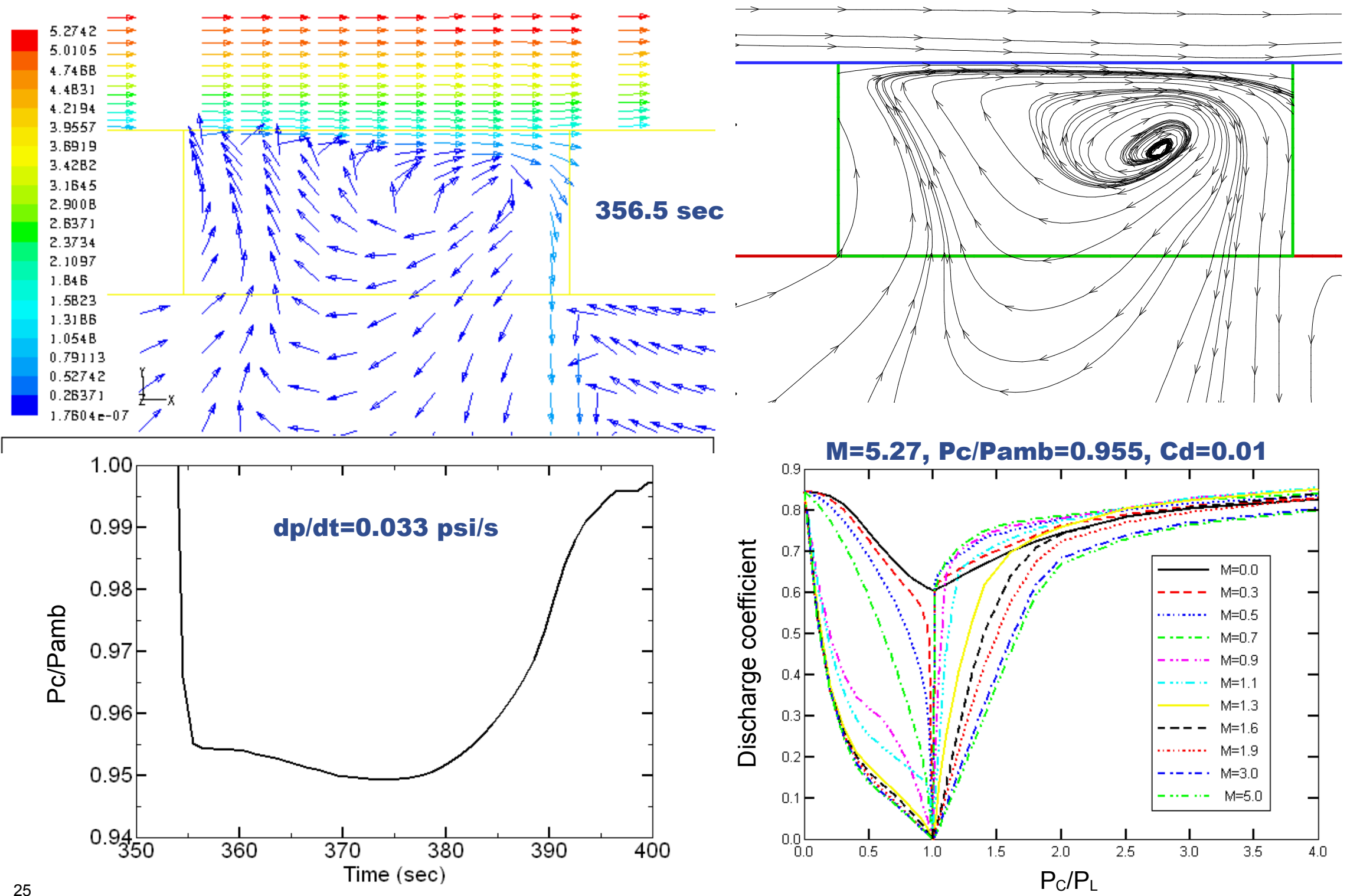


\section{Reentry: High Pressure Rising Rate}

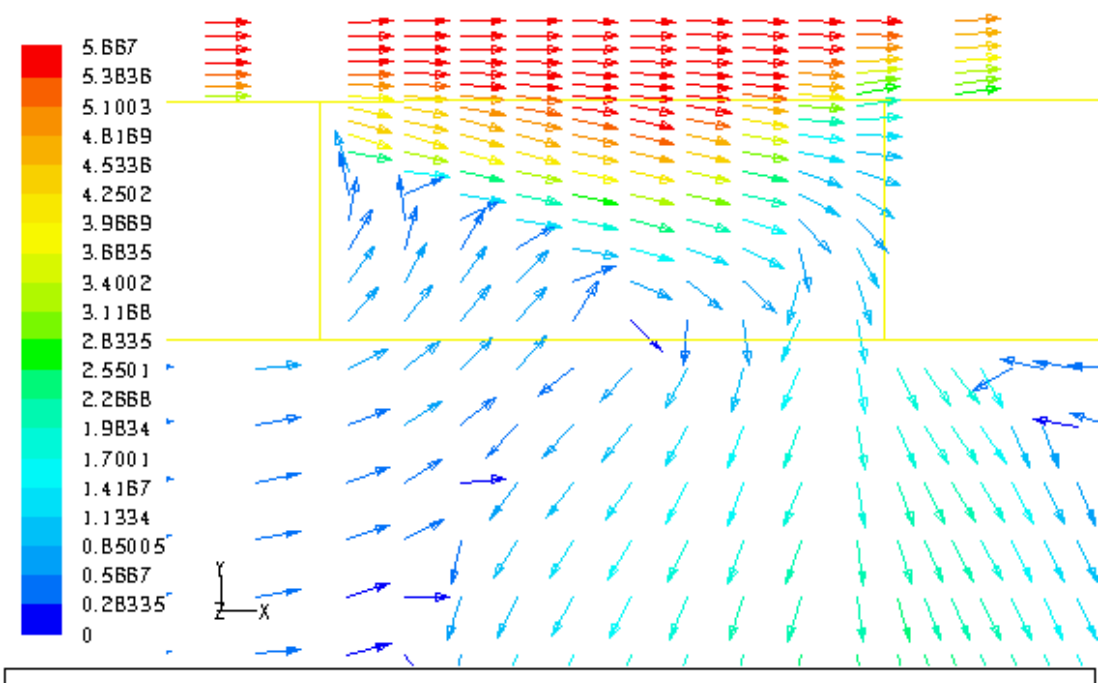

$\mathrm{dp} / \mathrm{dt}=25.3 \mathrm{psi} / \mathrm{s}, \mathrm{t}=354.16 \mathrm{sec} \quad \mathrm{Cd}=0.34$

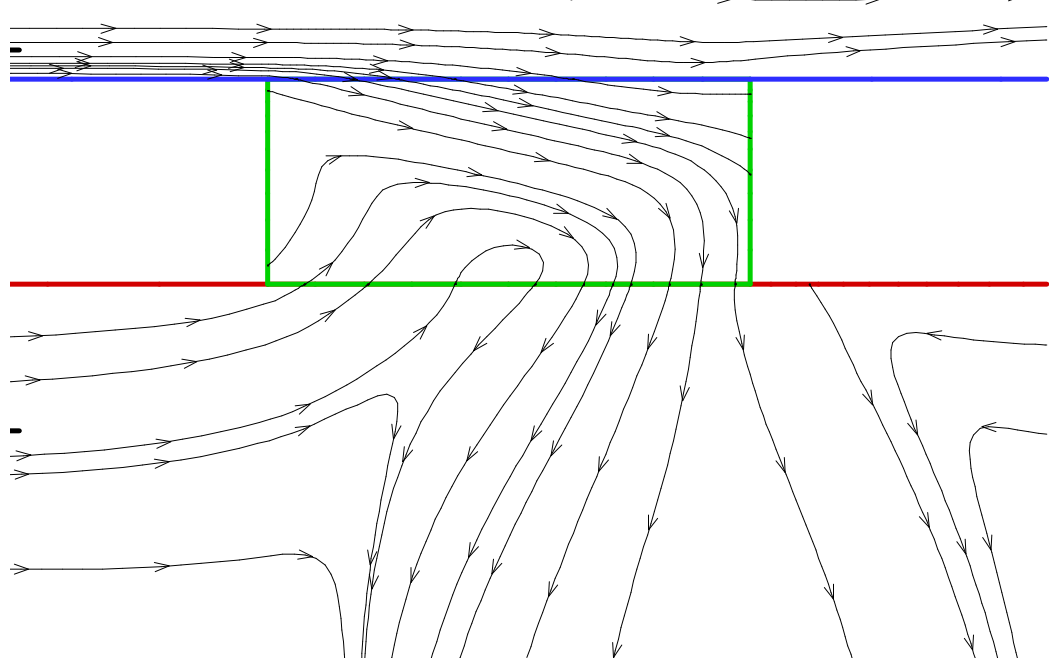

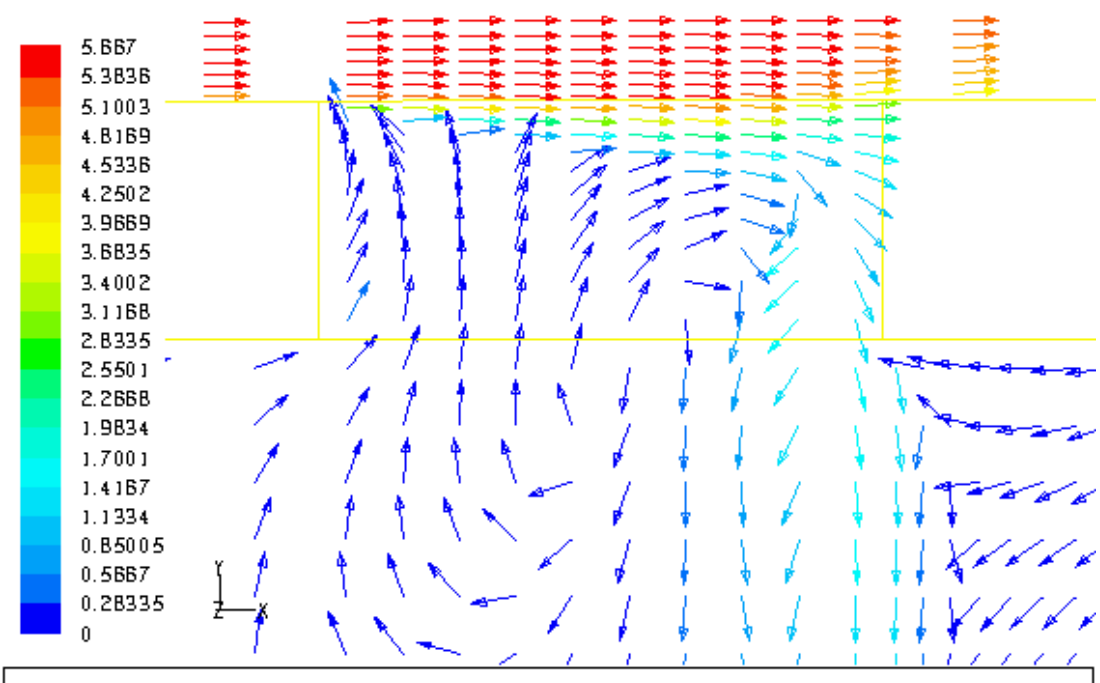

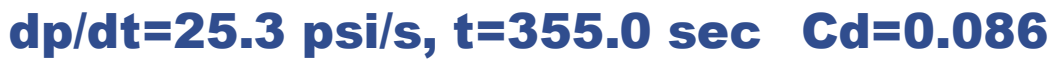

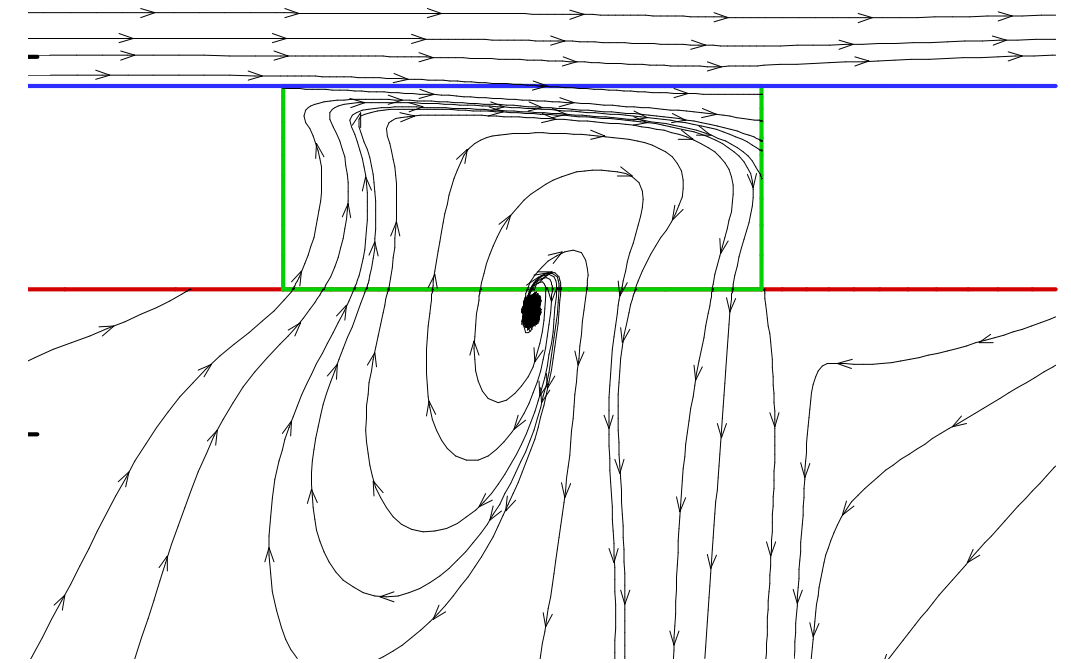




\section{Reentry: Velocity Vector and Streamlines}

\section{ATK}

A premier aerospace and defense company

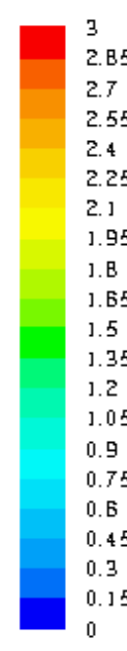

.

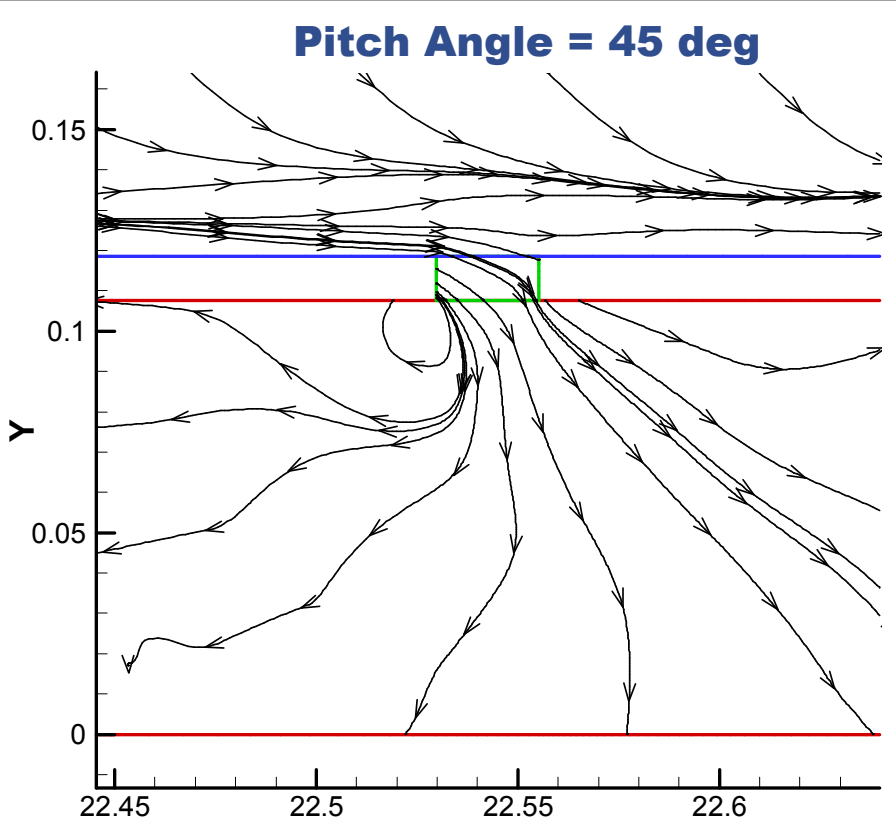

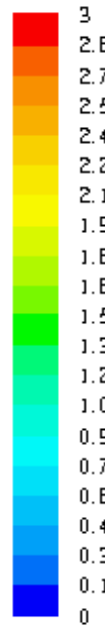

$E x$

Pitch Angle = $90 \mathrm{deg}$

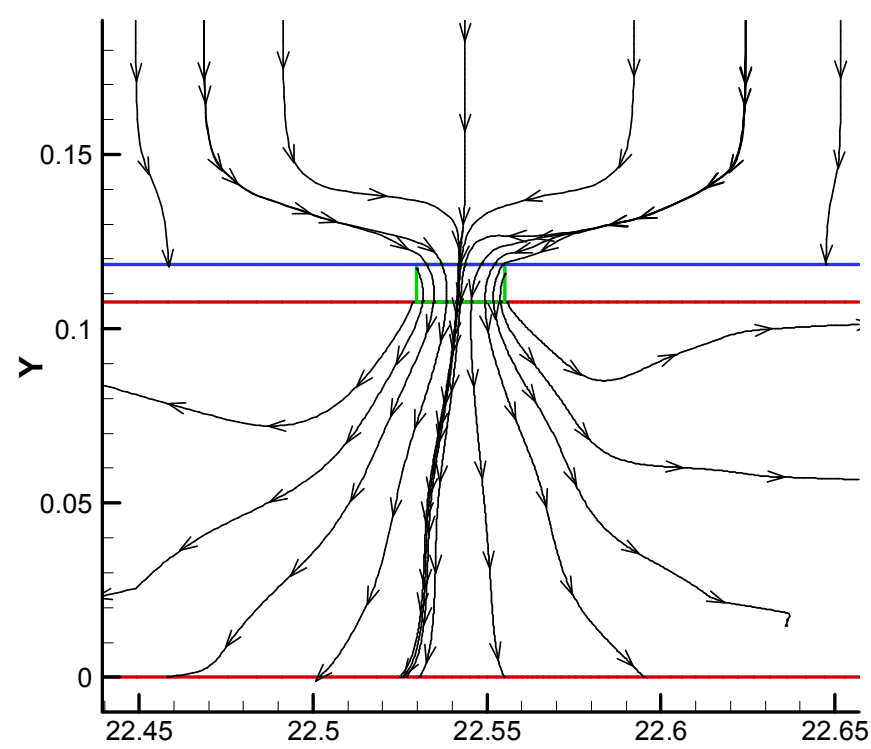




\section{FLUENT Versus CHCHVENT Predictions}

A premier aerospace and defense company
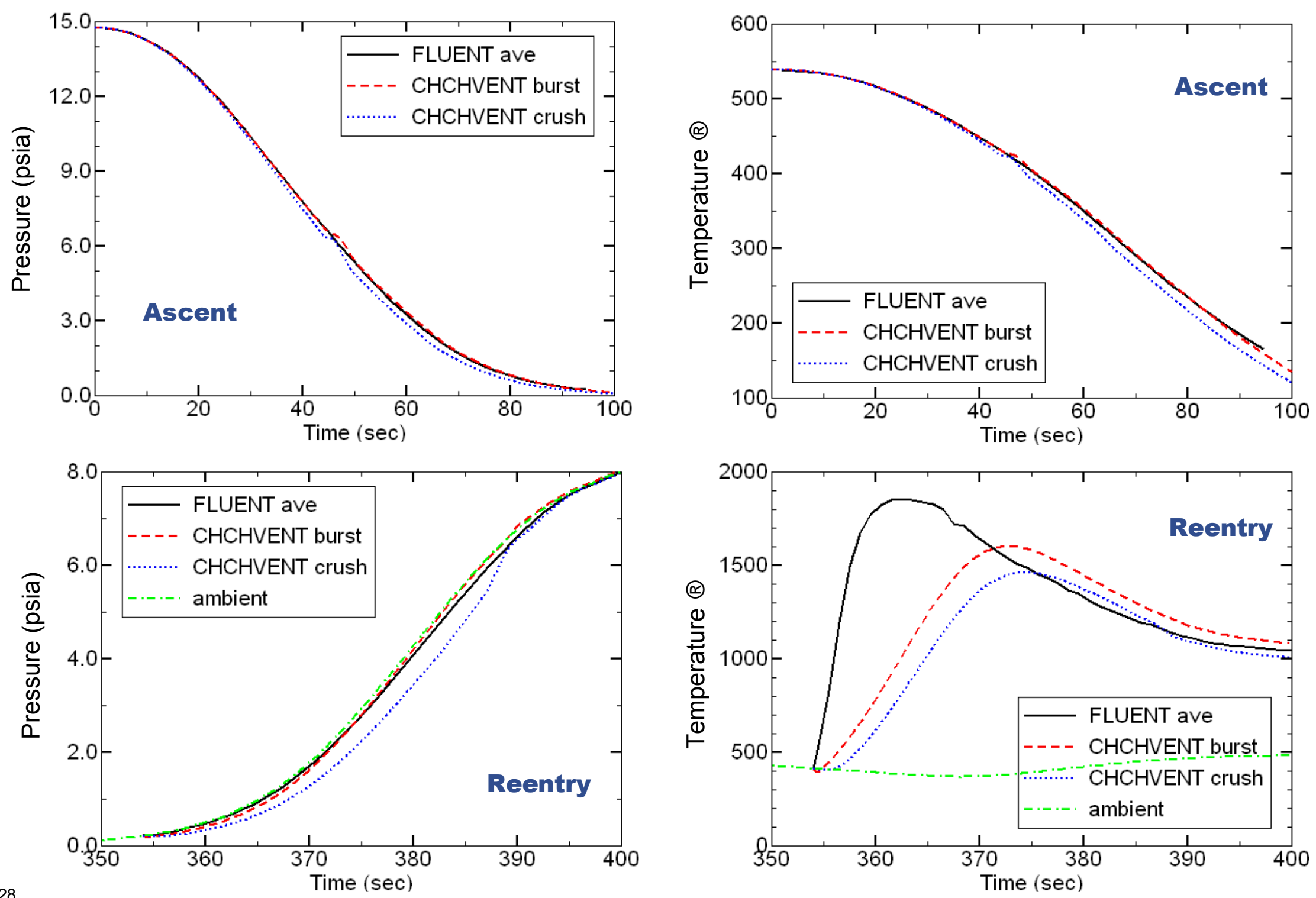


\section{Conclusions}

Performed Ares I first stage systems tunnel venting analyses during ascent and reentry

- Lumped parameter method using CHCHVENT

- 3D transient CFD approach using FLUENT

Uniform pressure and temperature assumptions inside the systems tunnel are valid during ascent

- The difference between maximum and minimum pressures is less than 0.01 psid

- Away from the vent, the difference is less than 0.0002 psid

- The difference between maximum and minimum temperatures is less than $0.7^{\circ} \mathrm{R}$

Uniform pressure assumption inside the tunnel is reasonable during reentry

- The difference between maximum and minimum pressures is less than 0.67 psid

- Away from the vent, the difference is less than 0.0013 psid

Uniform temperature assumption inside the tunnel is not valid during reentry

- The difference between maximum and minimum temperatures is as much as $3150{ }^{\circ} \mathrm{R}$ 


\section{Conclusions (Cont)}

The 95 percentile skin differential pressure

- During ascent: 1.04 psid for the burst case and -2.70 psid for the crush case

- During reentry: 9.37 for the burst case and -14.29 psid for the crush cases

The maximum pressure difference between compartment and ambient

- 0.75 psid during ascent

- 6.5 psid during reentry

Predicted average pressure and temperature inside the tunnel from FLUENT is between those for the burst and crush cases from CHCHVENT at most times

Velocity vectors and streamlines near the vent are reasonable

It takes much longer for 3D transient CFD than lumped parameter method

- FLUENT takes months on a Linux cluster with AMD Opteron $2.6 \mathrm{GHz}$ processors

- CHCHVENT takes minutes 